\title{
Asymmetric Total Syntheses of Aspidodasycarpine, Lonicerine, and the Proposed Structure of Lanciferine
}

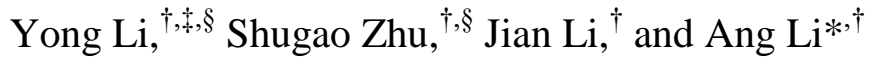 \\ ${ }^{\dagger}$ State Key Laboratory of Bioorganic and Natural Products Chemistry, Collaborative Innovation Center of \\ Chemistry for Life Sciences, Shanghai Institute of Organic Chemistry, Chinese Academy of Sciences, \\ 345 Lingling Road, Shanghai 200032, China

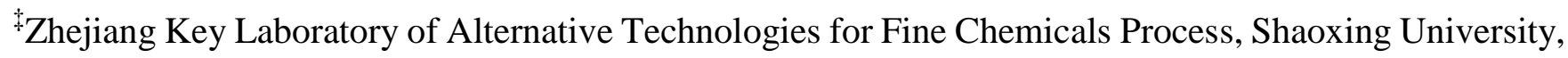 \\ Shaoxing 312000, China
}

Email: ali@sioc.ac.cn

I Experimental Procedures and Spectroscopic Data of Compounds

II References

III HPLC Traces for Measuring Enantiomeric Excess

IV ${ }^{1} \mathrm{H}$ and ${ }^{13} \mathrm{C}$ NMR Spectra of Compounds

V Comparison of the NMR Spectra and Data of Natural and Synthetic Aspidodasycarpine and Lonicerine 


\section{Experimental Procedures and Spectroscopic Data of Compounds}

General Procedures. All reactions were carried out under an argon atmosphere with dry solvents under anhydrous conditions, unless otherwise noted. Tetrahydrofuran (THF) and toluene were distilled immediately before use from sodium-benzophenone ketyl. Methylene chloride $\left(\mathrm{CH}_{2} \mathrm{Cl}_{2}\right)$, triethylamine $\left(\mathrm{Et}_{3} \mathrm{~N}\right), \mathrm{N}, \mathrm{N}$-dimethylformamide (DMF), dimethyl sulfoxide (DMSO), and acetonitrile (MeCN) were distilled from calcium hydride and stored under an argon atmosphere. Methanol (MeOH) was distilled from magnesium and stored under an argon atmosphere. Anhydrous isopropanol $(i$-PrOH) was purchased from Aldrich and used without further purification. Reagents were purchased at the highest commercial quality and used without further purification, unless otherwise stated. Solvents for chromatography were used as supplied by Titan chemical. Reactions were monitored by thin layer chromatography (TLC) carried out on S-2 $0.25 \mathrm{~mm}$ E. Merck silica gel plates (60F-254) using UV light as visualizing agent and aqueous ammonium cerium nitrate/ammonium molybdate or basic aqueous potassium permanganate as developing agent. E. Merck silica gel (60, particle size 0.040-0.063 mm) was used for flash column chromatography. Preparative thin layer chromatography separations were carried out on 0.25 or $0.50 \mathrm{~mm}$ E. Merck silica gel plates (60F-254). NMR spectra were recorded on Bruker AV-400, DRX-500, DRX-600, or Agilent 500/54/ASP instrument and calibrated by using residual undeuterated chloroform $\left(\delta_{\mathrm{H}}=7.26 \mathrm{ppm}\right)$ and $\mathrm{CDCl}_{3}\left(\delta_{\mathrm{C}}=77.16 \mathrm{ppm}\right)$ as internal references. The following abbreviations are used to designate multiplicities: $\mathrm{s}=$ singlet, $\mathrm{d}=$ doublet, $\mathrm{t}=$ triplet, $\mathrm{q}=$ quartet, $\mathrm{m}=$ multiplet, quint $=$ quintet, $\mathrm{br}=$ broad. $\mathrm{IR}$ spectra were recorded on a Thermo Scientific Nicolet 380 FT-IR spectrometer. Melting points (m.p.) are uncorrected and were recorded on a SGW X-4 apparatus. High-resolution mass spectra (HRMS) were recorded on a Bruker APEXIII 7.0 Tesla ESI-FT, a Waters Micromass GCT Premier EI, or an IonSpec 4.7 Tesla FT mass spectrometer. 


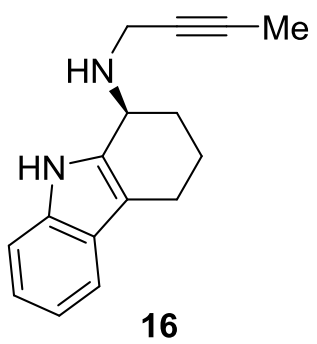

Secondary amine 16: To a stirred solution of 2,3,4,9-tetrahydro-1H-carbazol-1-one ${ }^{1}(\mathbf{1 4}, 12.8 \mathrm{~g}, 69.1$ mmol) and but-2-ynylamine $(\mathbf{1 3}, 8.95 \mathrm{~g}, 130 \mathrm{mmol})$ in $\mathrm{CH}_{2} \mathrm{Cl}_{2}(40 \mathrm{~mL})$ were added $4 \AA$ molecular sieves $(20 \mathrm{~g})$ and anhydrous $\mathrm{MgSO}_{4}(20 \mathrm{~g})$ at $40{ }^{\circ} \mathrm{C}$. The resultant mixture was stirred at that temperature for $2 \mathrm{~d}$ before $\mathrm{CH}_{2} \mathrm{Cl}_{2}(300 \mathrm{~mL})$ was added. The mixture so obtained was filtered and concentrated under vacuum, and the residue was dissolved in $\mathrm{CH}_{2} \mathrm{Cl}_{2}(70 \mathrm{~mL})$. To this solution were sequentially added water $(140 \mathrm{~mL}), \mathrm{HCO}_{2} \mathrm{Na}(29.4 \mathrm{~g}, 432 \mathrm{mmol}), \mathrm{Bu}_{4} \mathrm{NBr}(27.7 \mathrm{~g}, 85.9 \mathrm{mmol})$, and Noyori catalyst $15(2.75 \mathrm{~g}, 4.32 \mathrm{mmol})$ at $22{ }^{\circ} \mathrm{C}$. The reaction mixture was stirred at that temperature for $2 \mathrm{~d}$ before it was quenched with saturated aq. $\mathrm{Na}_{2} \mathrm{CO}_{3}(50 \mathrm{~mL})$ and extracted with $\mathrm{CH}_{2} \mathrm{Cl}_{2}(3 \times 150 \mathrm{~mL})$. The combined organic phases were washed with brine $(100 \mathrm{~mL})$, dried over anhydrous $\mathrm{Na}_{2} \mathrm{SO}_{4}$, and filtered. The solvent was evaporated under vacuum, and the residue was subjected to flash column chromatography for purification using EtOAc/petroleum ether (1:4) and then $\mathrm{Et}_{3} \mathrm{~N} / \mathrm{EtOAc} /$ petroleum ether/ (1:100:100) as eluent to give secondary amine $\mathbf{1 6}(11.2 \mathrm{~g}, 68 \%$ for the two steps, $95 \%$ ee) as a pale yellow oil. 16: $R_{\mathrm{f}}=0.36$ (silica, EtOAc:petroleum ether 1:2); $[\alpha]_{\mathrm{D}}^{24}=-32.2\left(c=0.20\right.$ in $\left.\mathrm{CHCl}_{3}\right)$; IR (film): $v_{\max }=3042,3299,3054,2850,1656,1454,1325,1155,798,740 \mathrm{~cm}^{-1} ;{ }^{1} \mathrm{H}$ NMR $(500 \mathrm{MHz}$, $\left.\mathrm{CDCl}_{3}\right): \delta=8.47(\mathrm{~s}, 1 \mathrm{H}), 7.49(\mathrm{~d}, J=7.8 \mathrm{~Hz}, 1 \mathrm{H}), 7.30(\mathrm{~d}, J=8.0 \mathrm{~Hz}, 1 \mathrm{H}), 7.15(\mathrm{t}, J=7.5 \mathrm{~Hz}, 1 \mathrm{H})$ 7.09 (t, $J=7.4 \mathrm{~Hz}, 1 \mathrm{H}), 4.09$ (dd, $J=6.6,5.7 \mathrm{~Hz}, 1 \mathrm{H}), 3.57$ (dq, $J=16.2,2.3 \mathrm{~Hz}, 1 \mathrm{H}), 3.51(\mathrm{dq}, J=$ 16.2, $2.3 \mathrm{~Hz}, 1 \mathrm{H}), 2.72(\mathrm{t}, J=6.1 \mathrm{~Hz}, 2 \mathrm{H}), 2.23(\mathrm{dddd}, J=12.8,7.8,5.1,2.5 \mathrm{~Hz}, 1 \mathrm{H}), 2.10-1.99$ (m, 1 $\mathrm{H}), 1.88(\mathrm{t}, J=2.3 \mathrm{~Hz}, 3 \mathrm{H}), 1.87-1.78(\mathrm{~m}, 1 \mathrm{H}), 1.69(\mathrm{dddd}, J=12.8,10.0,7.3,2.6 \mathrm{~Hz}, 1 \mathrm{H}) \mathrm{ppm} ;{ }^{13} \mathrm{C}$ $\operatorname{NMR}\left(126 \mathrm{MHz}, \mathrm{CDCl}_{3}\right): \delta=135.95,135.53,127.48,121.69,119.15,118.42,111.54,110.92,79.58$, 77.69, 51.38, 36.07, 30.25, 21.41, 21.08, $3.72 \mathrm{ppm}$; HRMS $(\mathrm{m} / \mathrm{z}):[\mathrm{M}+\mathrm{H}]^{+}$calcd for $\mathrm{C}_{16} \mathrm{H}_{18} \mathrm{~N}_{2}{ }^{+}$ 238.1470, found 238.1473 . 


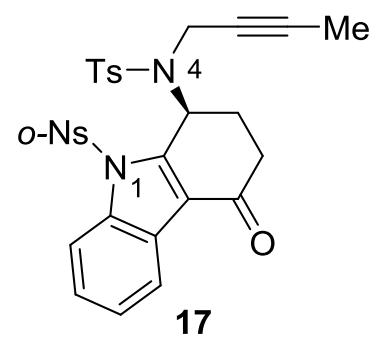

Ketone 17: To a stirred solution of secondary amine $\mathbf{1 6}(12.3 \mathrm{~g}, 51.6 \mathrm{mmol})$ in $\mathrm{CH}_{2} \mathrm{Cl}_{2}(300 \mathrm{~mL})$ were sequentially added $\mathrm{Et}_{3} \mathrm{~N}(8.60 \mathrm{~mL}, 6.24 \mathrm{~g}, 61.7 \mathrm{mmol})$ and $\mathrm{TsCl}(10.8 \mathrm{~g}, 56.8 \mathrm{mmol})$ at $0{ }^{\circ} \mathrm{C}$. The reaction mixture was stirred at that temperature for $1 \mathrm{~h}$ before it was quenched with saturated aq. $\mathrm{NaHCO}_{3}(300 \mathrm{~mL})$. The resultant mixture was extracted with $\mathrm{CH}_{2} \mathrm{Cl}_{2}(3 \times 300 \mathrm{~mL})$. The combined organic phases were washed with brine $(300 \mathrm{~mL})$, dried over anhydrous $\mathrm{Na}_{2} \mathrm{SO}_{4}$, filtered, and concentrated under vacuum. The residue was purified by flash column chromatography with EtOAc/petroleum ether $(1: 10 \rightarrow 1: 4)$ to give the $N 4$-Ts tetrahydrocarbazole $(16.8 \mathrm{~g}, 83 \%)$ as a white foam. This foam was dissolved in THF/water $(330 \mathrm{~mL}, 10: 1)$. To the stirred solution was added a solution of DDQ $(19.4 \mathrm{~g}, 85.6 \mathrm{mmol})$ in THF $(90 \mathrm{~mL})$ at $0{ }^{\circ} \mathrm{C}$. The resultant mixture was allowed to warm to $22{ }^{\circ} \mathrm{C}$ and stirred at that temperature for $8 \mathrm{~h}$ before it was quenched with saturated aq. $\mathrm{NaHCO}_{3}$ $(300 \mathrm{~mL})$ and extracted with EtOAc $(3 \times 300 \mathrm{~mL})$. The combined organic phases were washed with brine $(200 \mathrm{~mL})$ and dried over anhydrous $\mathrm{Na}_{2} \mathrm{SO}_{4}$. After filtration and evaporation of the solvent under vacuum, the residue was subjected to flash column chromatography for purification using EtOAc/petroleum ether $(1: 10 \rightarrow 1: 2)$ as eluent to give the $N 4-T$ s tricyclic ketone $(16.2 \mathrm{~g}, 93 \%)$ as a white foam. This foam was dissolved in $\mathrm{CH}_{2} \mathrm{Cl}_{2}(120 \mathrm{~mL})$. To the stirred solution were sequentially added $\mathrm{Et}_{3} \mathrm{~N}(28.0 \mathrm{~mL}, 20.3 \mathrm{~g}, 201 \mathrm{mmol}), 4-\mathrm{DMAP}(24.3 \mathrm{~g}, 199 \mathrm{mmol})$, and $o-\mathrm{NsCl}$ (44.2 g, $\left.199 \mathrm{mmol}\right)$ at $22{ }^{\circ} \mathrm{C}$. The resultant mixture was stirred at that temperature for $12 \mathrm{~h}$ and then quenched with saturated aq. $\mathrm{NaHCO}_{3}$ solution $(300 \mathrm{~mL})$. After extraction with $\mathrm{CH}_{2} \mathrm{Cl}_{2}(3 \times 300 \mathrm{~mL})$ and washing with brine $\left(300 \mathrm{~mL}\right.$ ), the combined organic phases were dried over anhydrous $\mathrm{Na}_{2} \mathrm{SO}_{4}$, filtered, and concentrated under vacuum. The residue was purified by flash column chromatography with EtOAc/petroleum ether $(1: 5 \rightarrow 1: 1)$ to give ketone $17(16.4 \mathrm{~g}, 70 \%)$ as a white foam. 17: $R_{\mathrm{f}}=0.42$ (silica, EtOAc:petroleum ether 1:1); $[\alpha]_{\mathrm{D}}^{21}=+58.0\left(c=0.60\right.$ in $\left.\mathrm{CHCl}_{3}\right)$; IR (film): $v_{\max }=2920,1675,1544,1448,1402,1356$, 
1184, 1090, 932, $752 \mathrm{~cm}^{-1} ;{ }^{1} \mathrm{H}$ NMR (400 MHz, $\left.\mathrm{CDCl}_{3}\right): \delta=8.39(\mathrm{dd}, J=7.2,1.2 \mathrm{~Hz}, 1 \mathrm{H}), 7.98(\mathrm{dd}, J$ $=7.8,1.3 \mathrm{~Hz}, 1 \mathrm{H}), 7.84(\mathrm{dd}, J=7.8,1.4 \mathrm{~Hz}, 1 \mathrm{H}), 7.80-7.70(\mathrm{~m}, 4 \mathrm{H}), 7.69(\mathrm{~d}, J=7.9 \mathrm{~Hz}, 1 \mathrm{H})$, 7.39-7.30 (m, $2 \mathrm{H}), 7.21(\mathrm{~d}, J=8.1 \mathrm{~Hz}, 2 \mathrm{H}), 5.89(\mathrm{t}, J=3.3 \mathrm{~Hz}, 1 \mathrm{H}), 3.99(\mathrm{dq}, J=18.6,2.3 \mathrm{~Hz}, 1 \mathrm{H})$, $3.88(\mathrm{dq}, J=18.6,2.2 \mathrm{~Hz}, 1 \mathrm{H}), 3.17-3.05(\mathrm{~m}, 1 \mathrm{H}), 2.84-2.74(\mathrm{~m}, 1 \mathrm{H}), 2.70-2.54$ (m, $2 \mathrm{H}), 2.39$ (s, 3 $\mathrm{H}), 1.45(\mathrm{t}, J=2.1 \mathrm{~Hz}, 3 \mathrm{H}) \mathrm{ppm} ;{ }^{13} \mathrm{C} \mathrm{NMR}\left(101 \mathrm{MHz}, \mathrm{CDCl}_{3}\right): \delta=195.13,147.35,146.12,143.54$, $137.06,135.73,135.69,133.04,131.59,130.98,129.26,127.81,126.59,125.86,125.48,125.23,123.07$, $121.92,113.70,81.32,73.83,50.72,37.41,34.40,30.06,21.46,3.15$ ppm; HRMS $(\mathrm{m} / z):[\mathrm{M}+\mathrm{H}]^{+}$calcd for $\mathrm{C}_{29} \mathrm{H}_{26} \mathrm{~N}_{3} \mathrm{O}_{7} \mathrm{~S}_{2}{ }^{+}$592.1207, found 592.1208.
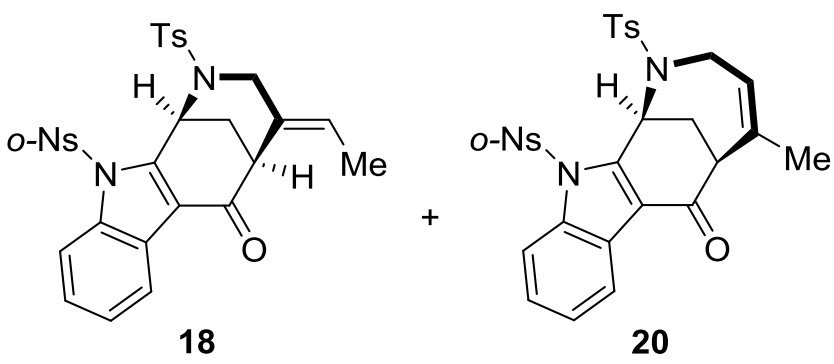

Cyclization products 18 and 20: To a stirred solution of ketone 17 (12.9 g, $21.7 \mathrm{mmol})$ in $\mathrm{CH}_{2} \mathrm{Cl}_{2}(100$ $\mathrm{mL})$ were sequentially added $\mathrm{Et}_{3} \mathrm{~N}(4.00 \mathrm{~mL}, 2.90 \mathrm{~g}, 28.7 \mathrm{mmol})$ and TIPSOTf $(6.45 \mathrm{~mL}, 7.35 \mathrm{~g}, 24.0$ $\mathrm{mmol}$ ) at $22{ }^{\circ} \mathrm{C}$. The reaction mixture was stirred at that temperature for $30 \mathrm{~min}$ before it was quenched with saturated aq. $\mathrm{NaHCO}_{3}(300 \mathrm{~mL})$. The resultant mixture was extracted with $\mathrm{CH}_{2} \mathrm{Cl}_{2}(3 \times 300 \mathrm{~mL})$, and the combined organic phase was washed with brine $(300 \mathrm{~mL})$, dried over anhydrous $\mathrm{Na}_{2} \mathrm{SO}_{4}$, and filtered. The solvent was removed under vacuum, and the residue was purified by flash column chromatography with EtOAc/petroleum ether $(1: 4 \rightarrow 1: 1)$ to give silyl enol ether $19(15.2 \mathrm{~g}, 94 \%)$ as a pale yellow oil. This oil was dissolved in $\mathrm{CH}_{2} \mathrm{Cl}_{2}(100 \mathrm{~mL})$. To the stirred solution were sequentially added $i$-PrOH (7.60 mL), pyrimidine 21 (504 mg, $2.03 \mathrm{mmol}), 4 \AA$ molecular sieves $(1.52 \mathrm{~g})$, $\left(4-\mathrm{CF}_{3} \mathrm{C}_{6} \mathrm{H}_{4}\right)_{3} \mathrm{PAuCl}^{2}(425 \mathrm{mg}, 0.609 \mathrm{mmol})$, and $\mathrm{AgSbF}_{6}(209 \mathrm{mg}, 0.609 \mathrm{mmol})$ at $22{ }^{\circ} \mathrm{C}$. The resultant mixture was allowed to stir at that temperature for $3 \mathrm{~d}$. The resultant mixture was directly subjected to flash column chromatography for purification using EtOAc/petroleum ether $(1: 4 \rightarrow 2: 1)$ as eluent to give cyclization products $\mathbf{1 8}(10.3 \mathrm{~g}, 86 \%)$ as a pale yellow powder and $\mathbf{2 0}(1.08 \mathrm{~g}, 9 \%)$ as a pale yellow foam. 18: $R_{\mathrm{f}}=0.59$ (silica, diethyl ether:petroleum ether 2:1); m.p.: 237-239 ${ }^{\circ} \mathrm{C}$ (EtOAc:hexane 1:1); [ $\left.\alpha\right]$ 
${ }_{\mathrm{D}}^{27}=+181.7\left(c=1.0\right.$ in $\left.\mathrm{CHCl}_{3}\right)$; IR (film): $v_{\max }=2963,1676,1544,1403,1354,1225,1160,943,751$, $716 \mathrm{~cm}^{-1} ;{ }^{1} \mathrm{H} \mathrm{NMR}\left(500 \mathrm{MHz}, \mathrm{CDCl}_{3}\right): \delta=8.30-8.25(\mathrm{~m}, 1 \mathrm{H}), 7.93(\mathrm{~d}, J=8.0 \mathrm{~Hz}, 1 \mathrm{H}), 7.90-7.84(\mathrm{~m}$, $1 \mathrm{H}), 7.80-7.75(\mathrm{~m}, 1 \mathrm{H}), 7.72(\mathrm{~d}, J=8.3 \mathrm{~Hz}, 2 \mathrm{H}), 7.68-7.62(\mathrm{~m}, 2 \mathrm{H}), 7.39-7.33(\mathrm{~m}, 2 \mathrm{H}), 7.21(\mathrm{~d}, J=$ $8.3 \mathrm{~Hz}, 2 \mathrm{H}), 6.20(\mathrm{~s}, 1 \mathrm{H}), 5.48(\mathrm{qd}, J=6.8,1.1 \mathrm{~Hz}, 1 \mathrm{H}), 3.94(\mathrm{~d}, J=16.4 \mathrm{~Hz}, 1 \mathrm{H}), 3.74(\mathrm{~s}, 1 \mathrm{H}), 3.51$ (d, $J=16.4 \mathrm{~Hz}, 1 \mathrm{H}), 2.54(\mathrm{ddd}, J=13.2,2.5,2.5 \mathrm{~Hz}, 1 \mathrm{H}), 2.37$ (s, $3 \mathrm{H}), 2.04$ (ddd, $J=13.2,3.3,3.3$ $\mathrm{Hz}, 1 \mathrm{H}), 1.69(\mathrm{dd}, J=6.8,1.8 \mathrm{~Hz}, 3 \mathrm{H}) \mathrm{ppm} ;{ }^{13} \mathrm{C} \mathrm{NMR}\left(126 \mathrm{MHz}, \mathrm{CDCl}_{3}\right): \delta=193.70,147.68,145.89$, $143.71,137.43,136.56,135.43,133.06,132.67,129.98,129.61,127.87,127.80,126.90,126.15,125.78$, $124.61,124.38,122.79,120.77,114.28,47.09,47.06,44.70,33.02,21.60,12.73$ ppm; HRMS $(m / z):[M$ $+\mathrm{Na}]^{+}$calcd for $\mathrm{C}_{29} \mathrm{H}_{25} \mathrm{~N}_{3} \mathrm{O}_{7} \mathrm{~S}_{2} \mathrm{Na}^{+}$614.1026, found 614.1031. CCDC 1406977 contains the supplementary crystallographic data of 18. These data can be obtained free of charge from The Cambridge Crystallographic Data Centre via www.ccdc.cam.ac.uk/data_request/cif. 20: $R_{\mathrm{f}}=0.61$ (silica, diethyl ether:petroleum ether 2:1); $[\alpha]_{\mathrm{D}}^{27}=+8.2\left(c=0.10\right.$ in $\left.\mathrm{CHCl}_{3}\right)$; IR (film): $v_{\max }=3098,2921,2855$, 1673, 1544, 1402, 1197, 1159, 873, $775 \mathrm{~cm}^{-1} ;{ }^{1} \mathrm{H}$ NMR $\left(400 \mathrm{MHz}, \mathrm{CDCl}_{3}\right): \delta=8.38-8.33(\mathrm{~m}, 1 \mathrm{H})$, 7.94-7.88 (m, $2 \mathrm{H}), 7.85(\mathrm{~d}, J=8.3 \mathrm{~Hz}, 2 \mathrm{H}), 7.81-7.75(\mathrm{~m}, 2 \mathrm{H}), 7.70(\mathrm{td}, J=7.8,1.3 \mathrm{~Hz}, 1 \mathrm{H})$, 7.40-7.32 (m, $2 \mathrm{H}), 7.26-7.21(\mathrm{~m}, 2 \mathrm{H}), 6.38(\mathrm{dd}, J=3.8,2.9 \mathrm{~Hz}, 1 \mathrm{H}), 5.56(\mathrm{~d}, J=6.1 \mathrm{~Hz}, 1 \mathrm{H}), 3.59$ $(\mathrm{dd}, J=18.0,7.6 \mathrm{~Hz}, 1 \mathrm{H}), 3.25(\mathrm{dd}, J=5.1,1.7 \mathrm{~Hz}, 1 \mathrm{H}), 3.07-2.97(\mathrm{~m}, 1 \mathrm{H}), 2.75-2.66(\mathrm{~m}, 1 \mathrm{H}), 2.53$ $(\mathrm{dt}, J=14.5,2.2 \mathrm{~Hz}, 1 \mathrm{H}), 2.39(\mathrm{~s}, 3 \mathrm{H}), 1.92(\mathrm{~s}, 3 \mathrm{H}) \mathrm{ppm} ;{ }^{13} \mathrm{C} \mathrm{NMR}\left(101 \mathrm{MHz}, \mathrm{CDCl}_{3}\right): \delta=190.25$, $147.73,146.67,143.65,140.04,137.45,136.17,135.54,132.87,132.31,130.85,129.52,128.24,126.91$, $126.22,125.74,125.29,125.22,123.20,122.76,114.09,51.96,49.57,41.54,32.59,27.73,21.67$; $\operatorname{HRMS}(m / z):[\mathrm{M}+\mathrm{Na}]^{+}$calcd for $\mathrm{C}_{29} \mathrm{H}_{25} \mathrm{~N}_{3} \mathrm{O}_{7} \mathrm{~S}_{2} \mathrm{Na}^{+}$614.1026, found 614.1020.

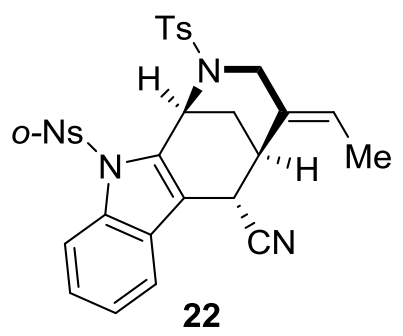

Nitrile 22: To a stirred solution of cyclization product 18 (8.69 g, $14.7 \mathrm{mmol})$ in $\mathrm{MeOH} / \mathrm{CH}_{2} \mathrm{Cl}_{2}(110$ $\mathrm{mL}, 10: 1)$ was added $\mathrm{NaBH}_{4}(667 \mathrm{mg}, 17.6 \mathrm{mmol})$ at $0{ }^{\circ} \mathrm{C}$. The reaction mixture was stirred at that 
temperature for 30 min before it was quenched with saturated aq. $\mathrm{NaHCO}_{3}(200 \mathrm{~mL})$. The resultant mixture was extracted with $\mathrm{CH}_{2} \mathrm{Cl}_{2}(3 \times 200 \mathrm{~mL})$. The combined organic phases were washed with brine $(100 \mathrm{~mL})$, dried over anhydrous $\mathrm{Na}_{2} \mathrm{SO}_{4}$, filtered, and concentrated under vacuum. The residue was purified by flash column chromatography with EtOAc/petroleum ether $(1: 1)$ to give the benzyl alcohol $(7.83 \mathrm{~g}, 90 \%)$ as a pale yellow foam. This foam was dissolved in $\mathrm{CH}_{2} \mathrm{Cl}_{2}(30 \mathrm{~mL})$. To the stirred solution were sequentially added TMSCN $(16.2 \mathrm{~mL}, 12.8 \mathrm{~g}, 131 \mathrm{mmol})$ and $\mathrm{BF}_{3} \cdot \mathrm{OEt}_{2}(12.6 \mathrm{~mL}, 14.5 \mathrm{~g}$, $102 \mathrm{mmol}$ ) at $22{ }^{\circ} \mathrm{C}$. The resultant mixture was allowed to stir at that temperature for $30 \mathrm{~min}$ before it was poured into saturated aq. $\mathrm{NaHCO}_{3}(200 \mathrm{~mL})$. The mixture so obtained was extracted with EtOAc (3 $\times 200 \mathrm{~mL})$, and the combined organic phases were washed with brine $(150 \mathrm{~mL})$, dried over anhydrous $\mathrm{Na}_{2} \mathrm{SO}_{4}$, and filtered. The solvent was evaporated under vacuum, and the residue was subjected to flash column chromatography for purification using EtOAc/petroleum ether $(1: 4 \rightarrow 2: 1)$ as eluent to give nitrile $22(6.03 \mathrm{~g}, 76 \%)$ as a white foam. 22: $R_{\mathrm{f}}=0.55$ (silica, EtOAc:petroleum ether 2:1); $[\alpha]_{\mathrm{D}}^{26}=$ $+145.9\left(c=0.10\right.$ in $\left.\mathrm{CHCl}_{3}\right)$; IR (film): $v_{\max }=3375,2924,2240,1732,1597,1544,1385,1354,1160$, 950, $737 \mathrm{~cm}^{-1} ;{ }^{1} \mathrm{H}$ NMR $\left(500 \mathrm{MHz}, \mathrm{CDCl}_{3}\right): \delta=7.94(\mathrm{~d}, J=7.5 \mathrm{~Hz}, 1 \mathrm{H}), 7.87(\mathrm{dd}, J=8.0,1.0 \mathrm{~Hz}, 1$ H), 7.74-7.69 (m, 3 H), 7.60-7.56 (m, 2 H), 7.51 (d, J=7.9 Hz, $1 \mathrm{H}), 7.41-7.34$ (m, $2 \mathrm{H}), 7.21$ (d, $J=$ $8.2 \mathrm{~Hz}, 2 \mathrm{H}), 6.02(\mathrm{dd}, J=2.8,2.8 \mathrm{~Hz}, 1 \mathrm{H}), 5.43(\mathrm{q}, J=6.8 \mathrm{~Hz}, 1 \mathrm{H}), 3.85(\mathrm{~s}, 1 \mathrm{H}), 3.82(\mathrm{~d}, J=16.3 \mathrm{~Hz}$, $1 \mathrm{H}), 3.52(\mathrm{~s}, 1 \mathrm{H}), 3.25$ (ddd, $J=16.3,1.9,1.8 \mathrm{~Hz}, 1 \mathrm{H}), 2.38$ (s, $3 \mathrm{H}), 2.38-2.34$ (m, $1 \mathrm{H}), 2.07$ (ddd, $J$ $=13.7,3.7,3.7 \mathrm{~Hz}, 1 \mathrm{H}), 1.65(\mathrm{dd}, J=6.8,1.9 \mathrm{~Hz}, 3 \mathrm{H}) \mathrm{ppm} ;{ }^{13} \mathrm{C} \mathrm{NMR}\left(101 \mathrm{MHz}, \mathrm{CDCl}_{3}\right): \delta=147.87$, $143.63,137.56,136.73,134.95,134.18,133.06,132.64,131.23,129.57,127.90,126.87,126.30,125.87$ $124.84,123.79,119.01,118.30,115.90,115.06,47.04,46.25,31.81,29.10,21.64,12.59$ ppm; HRMS $(m / z):[\mathrm{M}+\mathrm{Na}]^{+}$calcd for $\mathrm{C}_{30} \mathrm{H}_{26} \mathrm{~N}_{4} \mathrm{O}_{6} \mathrm{~S}_{2} \mathrm{Na}^{+}$625.1186, found 625.1182. 


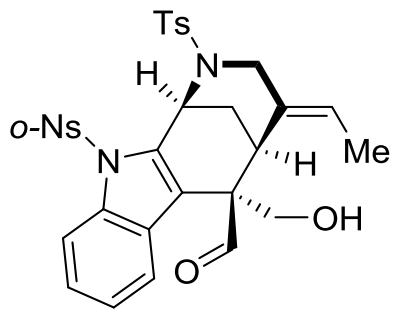

23

$\boldsymbol{\beta}$-Hydroxyaldehyde 23: To a stirred solution of nitrile $22(4.30 \mathrm{~g}, 7.14 \mathrm{mmol})$ in $\mathrm{CH}_{2} \mathrm{Cl}_{2}(200 \mathrm{~mL})$ was added DIBAL-H (14.3 $\mathrm{mL}, 1.0 \mathrm{M}$ in cyclohexane, $14.3 \mathrm{mmol})$ at $-78{ }^{\circ} \mathrm{C}$. The reaction mixture was allowed to stir at that temperature for $2 \mathrm{~h}$ before it was quenched with AcOH/diethyl ether (44 mL, 1:10) and diluted with brine $(100 \mathrm{~mL})$. The organic layer was separated, and the aqueous layer was extracted with EtOAc $(3 \times 100 \mathrm{~mL})$. The combined organic phases were washed with brine $(2 \times 100 \mathrm{~mL})$, dried over anhydrous $\mathrm{Na}_{2} \mathrm{SO}_{4}$, filtered, and concentrated under vacuum. The residue was passed through a plug of silica using EtOAc/petroleum ether $(1: 4 \rightarrow 1: 1)$ as eluent to give the aldehyde as a pale yellow foam. This foam was dissolved in $\mathrm{MeOH} / \mathrm{CH}_{2} \mathrm{Cl}_{2}(90 \mathrm{~mL}, 1: 2)$. To the stirred solution were sequentially added aq. $\mathrm{HCHO}(30 \mathrm{~mL}, 37 \mathrm{wt} \%)$ and $\mathrm{Na}_{2} \mathrm{CO}_{3}(2.62 \mathrm{~g}, 24.7 \mathrm{mmol})$ at $22{ }^{\circ} \mathrm{C}$. The resultant mixture was allowed to stir at that temperature for $3 \mathrm{~h}$ before it was quenched with saturated aq. $\mathrm{NaHCO}_{3}(100$ $\mathrm{mL})$ and extracted with $\mathrm{CH}_{2} \mathrm{Cl}_{2}(4 \times 100 \mathrm{~mL})$. The combined organic phases were sequentially washed with water $(2 \times 50 \mathrm{~mL})$ and brine $(2 \times 50 \mathrm{~mL})$, dried over anhydrous $\mathrm{Na}_{2} \mathrm{SO}_{4}$, and filtered. The solvent was evaporated under vacuum, and the residue was purified by flash column chromatography with EtOAc/petroleum ether $(1: 4 \rightarrow 2: 1)$ to give $\beta$-hydroxyaldehyde $23(2.49 \mathrm{~g}, 54 \%$ for the two steps $)$ as a white foam. 23: $R_{\mathrm{f}}=0.39$ (silica, EtOAc:petroleum ether 2:1); $[\alpha]_{\mathrm{D}}^{27}=+124.4\left(c=0.20\right.$ in $\left.\mathrm{CHCl}_{3}\right)$; $\mathrm{IR}$ (film): $v_{\max }=3524,2929,1719,1543,1450,1354,1159,1059,852,739 \mathrm{~cm}^{-1} ;{ }^{1} \mathrm{H} \mathrm{NMR}(400 \mathrm{MHz}$ $\left.\mathrm{CDCl}_{3}\right): \delta=9.63(\mathrm{~d}, J=1.2 \mathrm{~Hz}, 1 \mathrm{H}), 7.90(\mathrm{~d}, J=8.3 \mathrm{~Hz}, 1 \mathrm{H}), 7.84(\mathrm{~d}, J=7.6 \mathrm{~Hz}, 1 \mathrm{H}), 7.75-7.68(\mathrm{~m}$, $3 \mathrm{H}), 7.63-7.56(\mathrm{~m}, 2 \mathrm{H}), 7.35-7.27(\mathrm{~m}, 2 \mathrm{H}), 7.26-7.18(\mathrm{~m}, 3 \mathrm{H}), 6.03(\mathrm{t}, J=2.6 \mathrm{~Hz}, 1 \mathrm{H}), 5.45(\mathrm{q}, J=$ $6.8 \mathrm{~Hz}, 1 \mathrm{H}), 4.36(\mathrm{~d}, J=11.9 \mathrm{~Hz}, 1 \mathrm{H}), 3.89(\mathrm{~d}, J=16.1 \mathrm{~Hz}, 1 \mathrm{H}), 3.75(\mathrm{~d}, J=11.9 \mathrm{~Hz}, 1 \mathrm{H}), 3.70(\mathrm{~s}, 1$ H), $3.48(\mathrm{~d}, J=16.1 \mathrm{~Hz}, 1 \mathrm{H}), 2.75(\mathrm{~s}, 1 \mathrm{H}), 2.37(\mathrm{~s}, 3 \mathrm{H}), 2.25(\mathrm{~d}, J=13.7 \mathrm{~Hz}, 1 \mathrm{H}), 1.83(\mathrm{dt}, J=13.7$, $3.4 \mathrm{~Hz}, 1 \mathrm{H}), 1.52(\mathrm{dd}, J=6.8,1.8 \mathrm{~Hz}, 3 \mathrm{H}) \mathrm{ppm} ;{ }^{13} \mathrm{C} \mathrm{NMR}\left(126 \mathrm{MHz}, \mathrm{CDCl}_{3}\right): \delta=203.94,147.37$, $143.40,137.42,136.61,134.81,134.10,132.99,132.76,129.36,129.21,129.16,127.60,126.50,125.94$, 
$125.58,124.63,124.18,120.94,119.28,114.63,63.89,54.63,47.75,46.28,30.36,28.30,21.43,12.34$

ppm; HRMS $(m / z):[M+H]^{+}$calcd for $\mathrm{C}_{31} \mathrm{H}_{30} \mathrm{~N}_{3} \mathrm{O}_{8} \mathrm{~S}_{2}{ }^{+}$636.1469, found 636.1458.

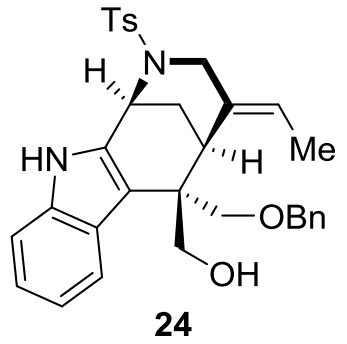

Primary Alcohol 24: To a stirred solution of $\beta$-hydroxyaldehyde 23 (2.12 g, 3.34 mmol) in 1,4-dioxane $(20 \mathrm{~mL})$ were sequentially added Yamada-Yu reagent ${ }^{3} \mathbf{2 5}(4.20 \mathrm{~g}, 15.1 \mathrm{mmol}), 4 \AA$ molecular sieves (700 mg), and $\mathrm{TfOH}(30.0 \mu \mathrm{L}, 50.1 \mathrm{mg}, 0.334 \mathrm{mmol})$ at $22^{\circ} \mathrm{C}$. The resultant mixture was allowed to stir at that temperature for $2 \mathrm{~h}$ before it was quenched with saturated aq. $\mathrm{NaHCO}_{3}(50 \mathrm{~mL})$ and diluted with brine $(50 \mathrm{~mL})$. The mixture so obtained was extracted with EtOAc $(3 \times 100 \mathrm{~mL})$, and the combined organic phases were washed with brine $(50 \mathrm{~mL})$ and dried over anhydrous $\mathrm{Na}_{2} \mathrm{SO}_{4}$. After filtration and evaporation of the solvent under vacuum, the residue was subjected to flash column chromatography for purification using EtOAc/petroleum ether $(1: 8 \rightarrow 1: 5)$ as eluent to give the $\beta$-benzyloxyaldehyde as a white foam. This foam was dissolved in $\mathrm{MeOH} / \mathrm{CH}_{2} \mathrm{Cl}_{2}(40 \mathrm{~mL}, 1: 1)$. To the stirred solution was added $\mathrm{NaBH}_{4}(151 \mathrm{mg}, 4.01 \mathrm{mmol})$ at $22{ }^{\circ} \mathrm{C}$. The reaction mixture was stirred at that temperature for $10 \mathrm{~min}$ before aq. $\mathrm{NaOH}(10 \mathrm{~mL}, 6.0 \mathrm{M})$ was added. To the resultant mixture were sequentially added DMF (20 $\mathrm{mL})$ and thioglycolic acid $(1.70 \mathrm{~mL}, 3.08 \mathrm{~g}, 33.4 \mathrm{mmol})$ at $0{ }^{\circ} \mathrm{C}$. The mixture so obtained was stirred at that temperature for $2 \mathrm{~h}$ before it was diluted with water $(50 \mathrm{~mL})$ and extracted with EtOAc $(3 \times 50 \mathrm{~mL})$. The combined organic phases were washed with brine $(2 \times 30 \mathrm{~mL})$, dried over anhydrous $\mathrm{Na}_{2} \mathrm{SO}_{4}$, filtered, and concentrated under vacuum. The residue was purified by flash column chromatography with EtOAc/petroleum ether $(1: 4 \rightarrow 1: 2)$ to give primary alcohol $24(1.14 \mathrm{~g}, 63 \%$ for the two steps $)$ as a white powder. 24: $R_{\mathrm{f}}=0.38$ (silica, EtOAc:petroleum ether 1:2); m.p.: 188-192 ${ }^{\circ} \mathrm{C}($ EtOAc:hexane 1:1); $[\alpha]_{\mathrm{D}}^{25}=+18.9\left(c=1.0\right.$ in $\left.\mathrm{CHCl}_{3}\right)$; IR (film): $v_{\max }=3060,2927,2861,1598,1454,1329,1157,1095,933$, $700 \mathrm{~cm}^{-1} ;{ }^{1} \mathrm{H}$ NMR $\left(400 \mathrm{MHz}, \mathrm{CDCl}_{3}\right): \delta=8.16(\mathrm{~s}, 1 \mathrm{H}), 7.75(\mathrm{~d}, J=8.3 \mathrm{~Hz}, 2 \mathrm{H}), 7.69(\mathrm{~d}, J=8.0 \mathrm{~Hz}$, $1 \mathrm{H}), 7.37-7.27(\mathrm{~m}, 7 \mathrm{H}), 7.16(\mathrm{t}, J=7.6 \mathrm{~Hz}, 1 \mathrm{H}), 7.05(\mathrm{t}, J=7.5 \mathrm{~Hz}, 1 \mathrm{H}), 5.54(\mathrm{q}, J=6.8 \mathrm{~Hz}, 1 \mathrm{H})$, 
$5.00(\mathrm{dd}, J=2.7,2.7 \mathrm{~Hz}, 1 \mathrm{H}), 4.61(\mathrm{~d}, J=12.0 \mathrm{~Hz}, 1 \mathrm{H}), 4.48(\mathrm{~d}, J=12.0 \mathrm{~Hz}, 1 \mathrm{H}), 4.44(\mathrm{dd}, J=11.1$, $4.0 \mathrm{~Hz}, 1 \mathrm{H}), 4.08(\mathrm{~d}, J=9.6 \mathrm{~Hz}, 1 \mathrm{H}), 3.84(\mathrm{~d}, J=13.5 \mathrm{~Hz}, 1 \mathrm{H}), 3.76(\mathrm{dd}, J=11.1,8.7 \mathrm{~Hz}, 1 \mathrm{H}), 3.59$ $(\mathrm{d}, J=9.6 \mathrm{~Hz}, 1 \mathrm{H}), 3.57-3.48(\mathrm{~m}, 2 \mathrm{H}), 2.83(\mathrm{dd}, J=8.2,4.6 \mathrm{~Hz}, 1 \mathrm{H}), 2.45(\mathrm{~s}, 3 \mathrm{H}), 2.06(\mathrm{ddd}, J=$ 13.0, 2.8, $2.8 \mathrm{~Hz}, 1 \mathrm{H}), 1.75(\mathrm{dd}, J=6.8,1.5 \mathrm{~Hz}, 3 \mathrm{H}), 1.73-1.67(\mathrm{~m}, 1 \mathrm{H}) \mathrm{ppm} ;{ }^{13} \mathrm{C} \mathrm{NMR}(126 \mathrm{MHz}$ $\left.\mathrm{CDCl}_{3}\right): \delta=143.53,137.99,137.84,136.31,132.83,130.81,129.81,128.57,127.91,127.66,127.26$ $125.00,124.39,122.44,120.87,119.74,113.06,111.76,74.57,73.55,68.00,49.51,46.99,44.77,31.96$, 29.70, 21.64, 13.44 ppm; HRMS $(m / z):[\mathrm{M}+\mathrm{Na}]^{+}$calcd for $\mathrm{C}_{32} \mathrm{H}_{34} \mathrm{~N}_{2} \mathrm{O}_{4} \mathrm{SNa}^{+}$565.2131, found 565.2136. CCDC 1052435 contains the supplementary crystallographic data of $\mathbf{2 4}$. These data can be obtained free of charge from The Cambridge Crystallographic Data Centre via www.ccdc.cam.ac.uk/data_request/cif.

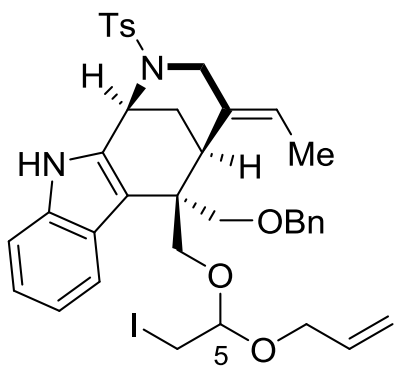

26

Iodoacetal 26: To a stirred solution of primary alcohol 24 (232 mg, $0.428 \mathrm{mmol})$ in $\mathrm{CH}_{2} \mathrm{Cl}_{2}(4.0 \mathrm{~mL})$ were sequentially added allyl vinyl ether $(2.0 \mathrm{~mL}), \mathrm{NaHCO}_{3}(287 \mathrm{mg}, 3.42 \mathrm{mmol})$, and NIS (785 mg, $3.49 \mathrm{mmol}$ ) at $0{ }^{\circ} \mathrm{C}$. The reaction mixture was stirred at that temperature for $30 \mathrm{~min}$ and then quenched with saturated aq. $\mathrm{Na}_{2} \mathrm{~S}_{2} \mathrm{O}_{3}(10 \mathrm{~mL})$. The combined organic phases were extracted with $\mathrm{CH}_{2} \mathrm{Cl}_{2}(3 \times 20$ $\mathrm{mL})$, washed with brine $(20 \mathrm{~mL})$, and dried over anhydrous $\mathrm{Na}_{2} \mathrm{SO}_{4}$. After filtration and removal of the solvent under vacuum, the residue was purified by flash column chromatography with EtOAc/petroleum ether $(1: 10 \rightarrow 1: 2)$ to give iodoacetal $26\left(302 \mathrm{mg}, 94 \%\right.$, ca. $1: 1 \mathrm{dr}$ at C5) as a white foam. 26: $R_{\mathrm{f}}=0.53$ (silica, EtOAc:petroleum ether $1: 2) ;[\alpha]_{\mathrm{D}}^{22}=+36.5\left(c=1.0\right.$ in $\left.\mathrm{CHCl}_{3}\right)$; IR (film): $v_{\max }=3388,3028$, 2927, 2861, 1712, 1494, 1329, 1158, 816, $735 \mathrm{~cm}^{-1} ;{ }^{1} \mathrm{H}$ NMR $\left(500 \mathrm{MHz}, \mathrm{CDCl}_{3}\right): \delta=8.18(\mathrm{~s}, 1 \mathrm{H})$, $8.14(\mathrm{~s}, 1 \mathrm{H}), 7.98(\mathrm{~d}, J=8.0 \mathrm{~Hz}, 1 \mathrm{H}), 7.89(\mathrm{~d}, J=8.1 \mathrm{~Hz}, 1 \mathrm{H}), 7.77(\mathrm{~d}, J=8.2 \mathrm{~Hz}, 4 \mathrm{H}), 7.36-7.21(\mathrm{~m}$, $16 \mathrm{H}), 7.16(\mathrm{t}, J=7.6 \mathrm{~Hz}, 2 \mathrm{H}), 7.08-7.00(\mathrm{~m}, 2 \mathrm{H}), 5.89-5.75(\mathrm{~m}, 2 \mathrm{H}), 5.50(\mathrm{q}, J=6.8 \mathrm{~Hz}, 2 \mathrm{H})$, 
5.22-5.15 (m, $2 \mathrm{H}), 5.15-5.11(\mathrm{~m}, 2 \mathrm{H}), 5.04(\mathrm{~d}, J=4.5 \mathrm{~Hz}, 2 \mathrm{H}), 4.71-4.63(\mathrm{~m}, 2 \mathrm{H}), 4.58(\mathrm{dd}, J=$ 15.5, $12.0 \mathrm{~Hz}, 2 \mathrm{H}), 4.45(\mathrm{~d}, J=12.0 \mathrm{~Hz}, 2 \mathrm{H}), 4.08-4.01(\mathrm{~m}, 2 \mathrm{H}), 4.00-3.89(\mathrm{~m}, 6 \mathrm{H}), 3.86(\mathrm{dd}, J=$ 13.6, 4.2 Hz, $2 \mathrm{H}), 3.80(\mathrm{~d}, J=9.4 \mathrm{~Hz}, 1 \mathrm{H}), 3.73(\mathrm{~d}, J=9.0 \mathrm{~Hz}, 1 \mathrm{H}), 3.67-3.53(\mathrm{~m}, 4 \mathrm{H}), 3.41(\mathrm{t}, J=$ $2.8 \mathrm{~Hz}, 1 \mathrm{H}), 3.34(\mathrm{t}, J=2.8 \mathrm{~Hz}, 1 \mathrm{H}), 3.24-3.12(\mathrm{~m}, 4 \mathrm{H}), 2.46(\mathrm{~s}, 6 \mathrm{H}), 2.22-2.10(\mathrm{~m}, 2 \mathrm{H}), 1.78-1.63$ $(\mathrm{m}, 8 \mathrm{H}) ;{ }^{13} \mathrm{C} \mathrm{NMR}\left(101 \mathrm{MHz}, \mathrm{CDCl}_{3}\right): \delta=143.53,138.59,138.53,138.01,138.00,136.26,136.25$, $134.15,134.13,133.01,132.88,130.49,130.47,129.82,128.35,128.34,127.70,127.55,127.26,125.81$, $125.79,123.93,123.66,122.59,122.54,122.35,122.33,119.37,119.31,117.21,117.17,114.48,114.40$, $111.33,111.27,101.97,101.88,73.39,73.28,73.04,72.14,70.05,69.85,67.17,66.90,49.41,49.34$, 47.08, 44.17, 43.97, 33.33, 33.02, 30.10, 30.00, 21.65, 13.44, 13.36, 5.23, 5.19 ppm; HRMS $(\mathrm{m} / \mathrm{z}):[\mathrm{M}]^{+}$ calcd for $\mathrm{C}_{37} \mathrm{H}_{41} \mathrm{IN}_{2} \mathrm{O}_{5} \mathrm{~S}^{+} 752.1781$, found 752.1771 .

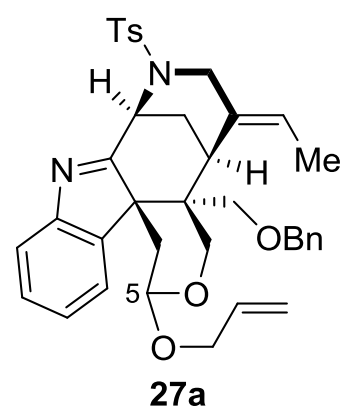

Cyclization product 27a: To a solution of iodoacetal $26(160 \mathrm{mg}, 0.213 \mathrm{mmol})$ in DMSO (12 mL) was added $t$-BuOLi (34.1 mg, $0.426 \mathrm{mmol})$. The resultant mixture was irradiated under microwave at $120{ }^{\circ} \mathrm{C}$ for $1 \mathrm{~h}$ before it was cooled to $22{ }^{\circ} \mathrm{C}$ and quenched with saturated aq. $\mathrm{NaHCO}_{3}(20 \mathrm{~mL})$. The mixture so obtained was extracted with EtOAc $(4 \times 20 \mathrm{~mL})$. The combined organic phases were sequentially washed with water $(20 \mathrm{~mL})$ and brine $(20 \mathrm{~mL})$, dried over anhydrous $\mathrm{Na}_{2} \mathrm{SO}_{4}$, filtered, and concentrated under vacuum. The residue was subjected to flash column chromatography for purification using EtOAc/petroleum ether $(1: 5 \rightarrow 1: 1)$ as eluent to give cyclization product $27 \mathbf{a}(96.7 \mathrm{mg}, 73 \%$, ca. $1.6: 1$ $\mathrm{dr}$ at $\mathrm{C} 5$ ) as a white foam. 27a: $R_{\mathrm{f}}=0.39$ (silica, EtOAc:petroleum ether $\left.1: 1\right) ;[\alpha]_{\mathrm{D}}^{26}=+34.7(c=1.0$ in $\mathrm{CHCl}_{3}$ ); IR (film): $v_{\max }=3438,2923,2855,1736,1646,1594,1351,1166,816,758 \mathrm{~cm}^{-1} ;{ }^{1} \mathrm{H} \mathrm{NMR}$ $\left(400 \mathrm{MHz}, \mathrm{CDCl}_{3}\right): \delta=7.97(\mathrm{~d}, J=7.3 \mathrm{~Hz}, 1 \mathrm{H}), 7.84-7.73(\mathrm{~m}, 4.24 \mathrm{H}), 7.65(\mathrm{~d}, J=7.2 \mathrm{~Hz}, 0.71 \mathrm{H})$ $7.62(\mathrm{~d}, J=7.2 \mathrm{~Hz}, 1.04 \mathrm{H}), 7.39-7.14(\mathrm{~m}, 11.55 \mathrm{H}), 7.11-7.02(\mathrm{~m}, 3.51 \mathrm{H}), 6.02-5.82(\mathrm{~m}, 1.68 \mathrm{H})$ 
$5.73(\mathrm{q}, J=6.7 \mathrm{~Hz}, 0.65 \mathrm{H}), 5.63(\mathrm{q}, J=6.9 \mathrm{~Hz}, 1 \mathrm{H}), 5.34-5.11(\mathrm{~m}, 3.33 \mathrm{H}), 4.94-4.86(\mathrm{~m}, 2.72 \mathrm{H})$, $4.75(\mathrm{dd}, J=4.1,2.3 \mathrm{~Hz}, 0.65 \mathrm{H}), 4.46-4.33(\mathrm{~m}, 1.77 \mathrm{H}), 4.29-3.87(\mathrm{~m}, 10.82 \mathrm{H}), 3.83-3.71$ (m, 1.28

H), $3.61(\mathrm{~s}, 1.04 \mathrm{H}), 3.38(\mathrm{~d}, J=9.6 \mathrm{~Hz}, 0.63 \mathrm{H}), 3.28(\mathrm{~s}, 0.65 \mathrm{H}), 2.95(\mathrm{dd}, J=15.1,5.0 \mathrm{~Hz}, 0.90 \mathrm{H})$, $2.76(\mathrm{~d}, J=10.1 \mathrm{~Hz}, 1 \mathrm{H}), 2.72(\mathrm{dd}, J=9.6,0.8 \mathrm{~Hz}, 0.67 \mathrm{H}), 2.49-2.37(\mathrm{~m}, 7.14 \mathrm{H}), 2.01-1.88(\mathrm{~m}, 1.07$ H), $1.85(\mathrm{t}, J=2.9 \mathrm{~Hz}, 1.84 \mathrm{H}), 1.81(\mathrm{~d}, J=6.9 \mathrm{~Hz}, 3.07 \mathrm{H}), 1.63(\mathrm{~d}, J=6.7 \mathrm{~Hz}, 2 \mathrm{H}), 1.54-1.40(\mathrm{~m}$, $1.36 \mathrm{H}), 1.27(\mathrm{~d}, J=15.1 \mathrm{~Hz}, 1 \mathrm{H}) \mathrm{ppm} ;{ }^{13} \mathrm{C} \mathrm{NMR}\left(101 \mathrm{MHz}, \mathrm{CDCl}_{3}\right): \delta=184.41,182.90,154.21$, $153.58,143.68,142.23,141.28,137.96,137.87,134.86,134.59,134.32,134.04,133.36,132.80,129.78$, $128.30,128.24,127.94,127.74,127.72,127.58,127.51,127.48,127.44,127.07,127.03,126.78,125.18$, $124.98,124.49,123.88,121.65,121.23,116.85,116.35,97.08,95.58,73.08,73.03,72.99,72.09,68.25$, $68.03,63.44,61.04,58.25,56.52,53.49,53.39,50.57,47.10,46.49,45.90,35.11,31.86,31.63,31.01$, 30.91, 30.30, 21.55, 14.43, 14.39 ppm; $\operatorname{HRMS}(\mathrm{m} / \mathrm{z}):[\mathrm{M}+\mathrm{H}]^{+}$calcd for $\mathrm{C}_{37} \mathrm{H}_{41} \mathrm{~N}_{2} \mathrm{O}_{5} \mathrm{~S}^{+} 625.2731$, found 625.2720.

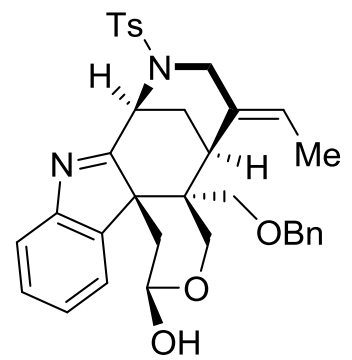

27

Lactol 27: A suspension of $\left[\operatorname{Ir}(\operatorname{cod})\left(\mathrm{MePPh}_{2}\right)_{2}\right] \mathrm{PF}_{6}{ }^{4}(8.0 \mathrm{mg}, 0.0092 \mathrm{mmol})$ in $\mathrm{THF}(0.50 \mathrm{~mL})$ was stirred under a hydrogen atmosphere at $22{ }^{\circ} \mathrm{C}$ for $10 \mathrm{~min}$. The orange-red suspension turned into a pale orange solution. This solution was purged with argon for $15 \mathrm{~min}$ before a solution of cyclization product 27 a $(58.6 \mathrm{mg}, 0.0938 \mathrm{mmol})$ in THF $(0.50 \mathrm{~mL})$ was added at $22{ }^{\circ} \mathrm{C}$. The reaction mixture was stirred at that temperature for $2.5 \mathrm{~h}$, and another portion of $\left[\operatorname{Ir}(\mathrm{cod})\left(\mathrm{MePPh}_{2}\right)_{2}\right] \mathrm{PF}_{6}(4.0 \mathrm{mg}, 0.0046 \mathrm{mmol})$ in $\mathrm{THF}$ $(0.20 \mathrm{~mL})$ that was pre-activated as described above was added. The resultant mixture was stirred at $22{ }^{\circ} \mathrm{C}$ for $2.5 \mathrm{~h}$ before the addition of aq. $\mathrm{HClO}_{4}(1.0 \mathrm{~mL}, 4.0 \mathrm{M})$. The mixture so obtained was stirred at that temperature for $2 \mathrm{~d}$ and then quenched with saturated aq. $\mathrm{NaHCO}_{3}(10 \mathrm{~mL})$ and extracted with EtOAc $(3 \times 20 \mathrm{~mL})$. The combined organic phases were dried over anhydrous $\mathrm{Na}_{2} \mathrm{SO}_{4}$ and filtered. The 
solvent was removed under vacuum, and the residue was purified by flash column chromatography with EtOAc/petroleum ether $(1: 4 \rightarrow 1: 1)$ to give lactol $27(40.7 \mathrm{mg}, 74 \%)$ as a white foam. $27: R_{\mathrm{f}}=0.15$ (silica, EtOAc:petroleum ether 1:2); $[\alpha]_{\mathrm{D}}^{25}=-4.2\left(c=1.0\right.$ in $\left.\mathrm{CHCl}_{3}\right)$; IR (film): $v_{\max }=2920,2850,1597$, $1462,1352,1275,1260,1165,764,750 \mathrm{~cm}^{-1} ;{ }^{1} \mathrm{H}$ NMR $\left(400 \mathrm{MHz}, \mathrm{CDCl}_{3}\right): \delta=7.81(\mathrm{~d}, J=8.2 \mathrm{~Hz}, 2$ H), 7.73 (d, $J=7.5 \mathrm{~Hz}, 1 \mathrm{H}), 7.66$ (d, $J=7.4 \mathrm{~Hz}, 1 \mathrm{H}), 7.37-7.30$ (m, $3 \mathrm{H}), 7.25-7.19$ (m, $4 \mathrm{H}), 7.16$ (td, $J=7.5,1.0 \mathrm{~Hz}, 1 \mathrm{H}), 7.07-7.02(\mathrm{~m}, 2 \mathrm{H}), 5.73(\mathrm{q}, J=6.8 \mathrm{~Hz}, 1 \mathrm{H}), 5.35-5.27(\mathrm{~m}, 1 \mathrm{H}), 4.74(\mathrm{t}, J=3.2$ $\mathrm{Hz}, 1 \mathrm{H}), 4.43(\mathrm{~d}, J=14.9 \mathrm{~Hz}, 1 \mathrm{H}), 4.30(\mathrm{~d}, J=12.1 \mathrm{~Hz}, 1 \mathrm{H}), 4.14(\mathrm{~d}, J=11.9 \mathrm{~Hz}, 1 \mathrm{H}), 3.98(\mathrm{~d}, J=$ $11.9 \mathrm{~Hz}, 1 \mathrm{H}), 3.86(\mathrm{~d}, J=12.1 \mathrm{~Hz}, 1 \mathrm{H}), 3.78-3.71(\mathrm{~m}, 1 \mathrm{H}), 3.31(\mathrm{~d}, J=9.6 \mathrm{~Hz}, 2 \mathrm{H}), 2.70(\mathrm{dd}, J=6.5$, $2.0 \mathrm{~Hz}, 1 \mathrm{H}), 2.47$ (dd, $J=14.3,8.3 \mathrm{~Hz}, 1 \mathrm{H}), 2.42(\mathrm{~s}, 3 \mathrm{H}), 2.00-1.89$ (m, $2 \mathrm{H}), 1.66$ (d, $J=6.8 \mathrm{~Hz}, 3$ H), $1.56(\mathrm{dd}, J=14.3,5.6 \mathrm{~Hz}, 1 \mathrm{H}) \mathrm{ppm} ;{ }^{13} \mathrm{C} \mathrm{NMR}\left(101 \mathrm{MHz}, \mathrm{CDCl}_{3}\right): \delta=184.26,154.21,143.88$, $141.22,138.02,134.07,133.55,129.94,128.38,128.15,127.81,127.60,127.19,126.65,125.16,124.73$, $121.82,92.18,73.09,72.96,62.18,58.67,53.58,50.27,46.57,36.65,31.28,30.39,21.67,14.57$; HRMS (m/z): $[\mathrm{M}+\mathrm{H}]^{+}$calcd for $\mathrm{C}_{34} \mathrm{H}_{37} \mathrm{~N}_{2} \mathrm{O}_{5} \mathrm{~S}^{+}$585.2418, found 585.2407.

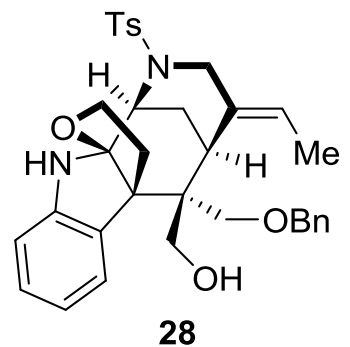

Pentacyclic alcohol 28: To a stirred solution of lactol $27(20.1 \mathrm{mg}, 0.0342 \mathrm{mmol})$ in $\mathrm{MeOH}(4.0 \mathrm{~mL})$ was added $\mathrm{NaBH}_{4}(12.9 \mathrm{mg}, 0.342 \mathrm{mmol})$ at $0{ }^{\circ} \mathrm{C}$. The reaction mixture was stirred at that temperature for $2 \mathrm{~h}$ before it was quenched with saturated aq. $\mathrm{NaHCO}_{3}(15 \mathrm{~mL})$. The resultant mixture was extracted with EtOAc $(3 \times 15 \mathrm{~mL})$. The combined organic phases were washed with brine $(15 \mathrm{~mL})$, dried over anhydrous $\mathrm{Na}_{2} \mathrm{SO}_{4}$, filtered, and concentrated under vacuum. The residue was subjected to flash column chromatography for purification using EtOAc/petroleum ether $(1: 4 \rightarrow 1: 1)$ as eluent to give pentacyclic alcohol $28(18.0 \mathrm{mg}, 90 \%)$ as a white powder. 28: $R_{\mathrm{f}}=0.28$ (silica, EtOAc:petroleum ether 1:1); $[\alpha]_{\mathrm{D}}^{25}=$ $-69.5\left(c=1.0\right.$ in $\left.\mathrm{CHCl}_{3}\right)$; IR (film): $v_{\max }=3525,3354,3031,2927,2874,1606,1595,1486,1468,1326$, 1156, 1098, 815, 747, $565 \mathrm{~cm}^{-1} ;{ }^{1} \mathrm{H}$ NMR (400 MHz, $\left.\mathrm{CDCl}_{3}\right): \delta=7.70(\mathrm{~d}, J=8.3 \mathrm{~Hz}, 2 \mathrm{H}), 7.33-7.25$ 
(m, $4 \mathrm{H}), 7.23(\mathrm{~d}, J=8.1 \mathrm{~Hz}, 2 \mathrm{H}), 7.18-7.12(\mathrm{~m}, 2 \mathrm{H}), 7.04$ (ddd, $J=7.7,7.7,1.0 \mathrm{~Hz}, 1 \mathrm{H}), 6.72$ (ddd, $J=7.6,7.5,0.6 \mathrm{~Hz}, 1 \mathrm{H}), 6.58(\mathrm{~d}, J=7.7 \mathrm{~Hz}, 1 \mathrm{H}), 5.54(\mathrm{q}, J=6.9 \mathrm{~Hz}, 1 \mathrm{H}), 4.46-4.39(\mathrm{~m}, 2 \mathrm{H}), 4.18$ $(\mathrm{d}, J=11.8 \mathrm{~Hz}, 1 \mathrm{H}), 4.12(\mathrm{~d}, J=11.8 \mathrm{~Hz}, 1 \mathrm{H}), 3.93(\mathrm{~d}, J=15.0 \mathrm{~Hz}, 1 \mathrm{H}), 3.86-3.73(\mathrm{~m}, 3 \mathrm{H}), 3.48$ $(\mathrm{dd}, J=8.0,8.0 \mathrm{~Hz}, 1 \mathrm{H}), 3.31-3.26(\mathrm{~m}, 2 \mathrm{H}), 3.21(\mathrm{ddd}, J=11.3,8.2,5.5 \mathrm{~Hz}, 1 \mathrm{H}), 2.93(\mathrm{~d}, J=10.0$ $\mathrm{Hz}, 1 \mathrm{H}), 2.50(\mathrm{ddd}, J=12.0,12.0,8.0 \mathrm{~Hz}, 1 \mathrm{H}), 2.38(\mathrm{~s}, 2 \mathrm{H}), 2.26(\mathrm{dd}, J=12.6,5.3 \mathrm{~Hz}, 1 \mathrm{H})$, $2.19-2.11(\mathrm{~m}, 2 \mathrm{H}), 1.66(\mathrm{dd}, J=6.9,1.7 \mathrm{~Hz}, 3 \mathrm{H}), 1.54(\mathrm{ddd}, J=13.8,3.6,3.6 \mathrm{~Hz}, 1 \mathrm{H}) \mathrm{ppm} ;{ }^{13} \mathrm{C}$ NMR (126 MHz, $\left.\mathrm{CDCl}_{3}\right): \delta=148.47,142.59,138.17,137.82,131.16,130.77,129.18,128.46,128.14$, $127.76,127.67,127.57,126.88,125.22,119.27,109.22,102.71,73.07,71.70,67.73,64.82,57.32$, 53.65, 48.60, 47.12, 35.76, 29.87, 27.07, 21.58, 13.57 ppm; HRMS $(m / z):[\mathrm{M}+\mathrm{H}]^{+}$calcd for $\mathrm{C}_{34} \mathrm{H}_{39} \mathrm{~N}_{2} \mathrm{O}_{5} \mathrm{~S}^{+}$587.2574, found 587.2566.

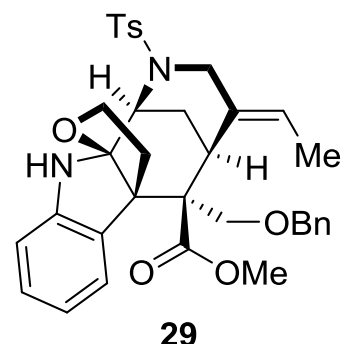

Pentacyclic methyl ester 29: To a stirred solution of pentacyclic alcohol 28 (36.0 $\mathrm{mg}, 0.0616 \mathrm{~mol})$ in $\mathrm{CH}_{2} \mathrm{Cl}_{2} /$ methyl-2-butene $(4.8 \mathrm{~mL}, 5: 1)$ were sequentially added aq. AcOH/NaOAc buffer $(1.0 \mathrm{M}, \mathrm{pH}=$ 4, $4.0 \mathrm{~mL}), \operatorname{AZADO}(9.2 \mathrm{mg}, 0.061 \mathrm{mmol})$, and $\mathrm{NaClO}_{2}(28.0 \mathrm{mg}, 0.308 \mathrm{mmol})$ at $22{ }^{\circ} \mathrm{C}^{5}$ The reaction mixture was stirred at that temperature for $10 \mathrm{~h}$ before it was quenched with saturated aq. $\mathrm{NaHSO}_{3}(10$ $\mathrm{mL})$ and extracted with $\mathrm{CHCl}_{3}(4 \times 10 \mathrm{~mL})$. The combined organic phases were washed with brine $(10$ $\mathrm{mL}$ ), dried over anhydrous $\mathrm{Na}_{2} \mathrm{SO}_{4}$, and filtered. The volatile was removed under vacuum, and the residue (crude carboxylic acid) was dissolved in $\mathrm{MeOH}(1.5 \mathrm{~mL})$. To the stirred solution was added $\mathrm{TMSCHN}_{2}(100 \mu \mathrm{L}, 2.0 \mathrm{M}$ in hexanes$)$ at $0{ }^{\circ} \mathrm{C}$. The resultant mixture was stirred at that temperature for 10 min before it was quenched with $\mathrm{AcOH}(50 \mu \mathrm{L})$. The mixture so obtained was concentrated under vacuum, and the residue was purified by flash column chromatography with EtOAc/petroleum ether $(1: 2 \rightarrow 1: 1)$ to give pentacyclic methyl ester $29(30.2 \mathrm{mg}, 80 \%$ for the two steps $)$ as a white foam. 29: $R_{\mathrm{f}}$ $=0.69$ (silica, EtOAc:petroleum ether 1:1); $[\alpha]_{\mathrm{D}}^{27}=-74.0\left(c=0.50\right.$ in $\left.\mathrm{CHCl}_{3}\right)$; IR (film): $v_{\max }=3387$, 
3054, 2917, 2849, 1720, 1608, 1327, 1265, 1158, 1100, 809, 740, $705 \mathrm{~cm}^{-1} ;{ }^{1} \mathrm{H}$ NMR $(400 \mathrm{MHz}$, $\left.\mathrm{CDCl}_{3}\right): \delta=7.70(\mathrm{~d}, J=8.3 \mathrm{~Hz}, 2 \mathrm{H}), 7.42(\mathrm{~d}, J=7.5 \mathrm{~Hz}, 1 \mathrm{H}), 7.31-7.19(\mathrm{~m}, 5 \mathrm{H}), 7.17-7.11(\mathrm{~m}, 2 \mathrm{H})$, 7.09 (ddd, $J=7.7,7.7,1.1 \mathrm{~Hz}, 1 \mathrm{H}), 6.77(\mathrm{ddd}, J=7.5,7.5,0.8 \mathrm{~Hz}, 1 \mathrm{H}), 6.60(\mathrm{~d}, J=7.7 \mathrm{~Hz}, 1 \mathrm{H}), 5.55$ (q, $J=6.8 \mathrm{~Hz}, 1 \mathrm{H}), 4.41-4.34(\mathrm{~m}, 2 \mathrm{H}), 4.25-4.14(\mathrm{~m}, 2 \mathrm{H}), 3.94(\mathrm{~d}, J=14.5 \mathrm{~Hz}, 1 \mathrm{H}), 3.79(\mathrm{~d}, J=$ $14.5 \mathrm{~Hz}, 1 \mathrm{H}), 3.76-3.66$ (m, $1 \mathrm{H}), 3.70$ (s, $3 \mathrm{H}), 3.53-3.41$ (m, $2 \mathrm{H}), 3.27$ (ddd, $J=10.9,8.5,6.2 \mathrm{~Hz}, 1$ H), $3.10(\mathrm{~d}, J=9.5 \mathrm{~Hz}, 1 \mathrm{H}), 2.45-2.35(\mathrm{~m}, 1 \mathrm{H}), 2.39$ (s, $3 \mathrm{H}), 2.31$ (dd, $J=12.7,5.8 \mathrm{~Hz}, 1 \mathrm{H}), 2.13$ (ddd, $J=13.9,2.9,2.9 \mathrm{~Hz}, 1 \mathrm{H}), 1.69(\mathrm{dd}, J=6.8,1.7 \mathrm{~Hz}, 3 \mathrm{H}), 1.55(\mathrm{ddd}, J=13.9,3.5,3.5 \mathrm{~Hz}, 1 \mathrm{H})$ ppm; ${ }^{13} \mathrm{C}$ NMR $\left(126 \mathrm{MHz}, \mathrm{CDCl}_{3}\right): \delta=173.74,148.37,142.59,138.23,138.03,132.15,129.93,129.20$, $128.75,128.31,127.64,127.52,127.19,126.98,125.51,119.67,109.52,102.97,73.63,72.99,67.71$, 56.44, 56.39, 53.61, 51.61, 49.78, 38.75, 29.87, 28.16, 21.61, 13.72 ppm; HRMS $(m / z):[\mathrm{M}+\mathrm{H}]^{+}$calcd for $\mathrm{C}_{35} \mathrm{H}_{39} \mathrm{O}_{6} \mathrm{~N}_{2} \mathrm{~S}^{+}$615.2523, found 615.2512.

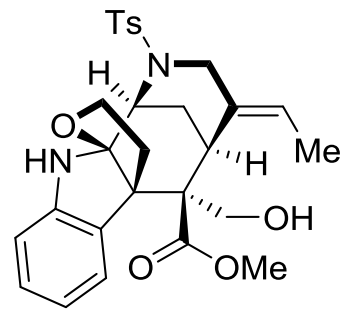

4a: N4-Ts-aspidodasycarpine

N4-Ts-aspidodasycarpine (4a): To a stirred solution of pentacyclic methyl ester 29 (14.0 mg, 0.0224 mmol $)$ in $\mathrm{CH}_{2} \mathrm{Cl}_{2}(2.0 \mathrm{~mL})$ was added $\mathrm{BBr}_{3}\left(200 \mu \mathrm{L}, 1.0 \mathrm{M}\right.$ in $\left.\mathrm{CH}_{2} \mathrm{Cl}_{2}, 0.200 \mathrm{mmol}\right)$ at $-78{ }^{\circ} \mathrm{C}$. The resultant mixture was stirred at that temperature for $1 \mathrm{~h}$ before it was quenched with saturated aq. $\mathrm{NaHCO}_{3}(5 \mathrm{~mL})$ and extracted with $\mathrm{CH}_{2} \mathrm{Cl}_{2}(3 \times 10 \mathrm{~mL})$. The combined organic phases were washed with brine $(5 \mathrm{~mL})$, dried over anhydrous $\mathrm{Na}_{2} \mathrm{SO}_{4}$, filtered, and concentrated under vacuum. The residue was passed through a short plug of silica using EtOAc/petroleum ether $(1: 3 \rightarrow 1: 1)$ as eluent to give N4-Ts-aspidodasycarpine (4a, $9.2 \mathrm{mg}, 78 \%$ ) as a colorless oil. $4 \mathrm{a}: R_{\mathrm{f}}=0.30$ (silica, EtOAc:petroleum ether 1:1); $[\alpha]_{\mathrm{D}}^{24}=-92.6\left(\mathrm{c}=0.22\right.$ in $\left.\mathrm{CHCl}_{3}\right)$; IR (film): $v_{\max }=3514,3354,2962,2925,2880,1746$, $1607,1597,1496,1470,1324,1263,1156,1099,738,546 \mathrm{~cm}^{-1} ;{ }^{1} \mathrm{H}$ NMR $\left(400 \mathrm{MHz}, \mathrm{CDCl}_{3}\right): \delta=7.70$ $(\mathrm{d}, J=8.3 \mathrm{~Hz}, 2 \mathrm{H}), 7.31$ (d, $J=7.5 \mathrm{~Hz}, 1 \mathrm{H}), 7.24(\mathrm{~d}, J=8.2 \mathrm{~Hz}, 2 \mathrm{H}), 7.09$ (ddd, $J=7.7,7.7,1.0 \mathrm{~Hz}$, $1 \mathrm{H}), 6.79(\mathrm{dd}, J=7.5,7.5 \mathrm{~Hz}, 1 \mathrm{H}), 6.63(\mathrm{~d}, J=7.8 \mathrm{~Hz}, 1 \mathrm{H}), 5.53(\mathrm{q}, J=6.8 \mathrm{~Hz}, 1 \mathrm{H}), 4.50(\mathrm{~s}, 1 \mathrm{H})$, 
$4.43(\mathrm{dd}, J=3.1,3.1 \mathrm{~Hz}, 1 \mathrm{H}), 3.94(\mathrm{~d}, J=14.8 \mathrm{~Hz}, 1 \mathrm{H}), 3.87(\mathrm{~d}, J=14.8 \mathrm{~Hz}, 1 \mathrm{H}), 3.74(\mathrm{~s}, 3 \mathrm{H})$,

3.60-3.53 (m, $2 \mathrm{H}), 3.54-3.46(\mathrm{~m}, 1 \mathrm{H}), 3.30$ (ddd, $J=8.5,8.5,8.4 \mathrm{~Hz}, 1 \mathrm{H}), 3.23-3.14(\mathrm{~m}, 1 \mathrm{H})$, 2.58-2.52 (m, $1 \mathrm{H}), 2.39(\mathrm{~s}, 3 \mathrm{H}), 2.23(\mathrm{ddd}, J=14.0,2.9,2.9 \mathrm{~Hz}, 1 \mathrm{H}), 1.66(\mathrm{~d}, J=6.8 \mathrm{~Hz}, 3 \mathrm{H})$, $1.64-1.57(\mathrm{~m}, J=3.5 \mathrm{~Hz}, 1 \mathrm{H}) \mathrm{ppm} ;{ }^{13} \mathrm{C} \mathrm{NMR}\left(126 \mathrm{MHz}, \mathrm{CDCl}_{3}\right): \delta=173.87,148.36,142.74,137.77$, $131.15,130.54,129.26,129.03,127.69,125.83,125.81,119.98,110.23,103.09,68.16,66.89,57.40$, 56.28, 53.39, 51.88, 49.15, 38.57, 30.93, 27.92, 21.63, $13.52 \mathrm{ppm}$; HRMS $(\mathrm{m} / \mathrm{z}):[\mathrm{M}+\mathrm{Na}]^{+}$calcd for $\mathrm{C}_{28} \mathrm{H}_{32} \mathrm{~N}_{2} \mathrm{O}_{6} \mathrm{SNa}^{+}$547.1873, found 547.1881.

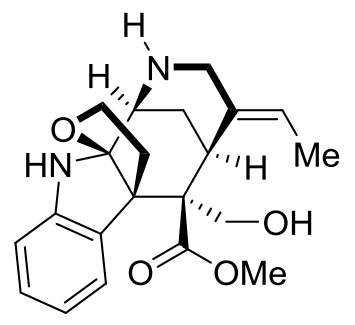

4: aspidodasycarpine

Aspidodasycarpine (4): To a stirred solution of N4-Ts-aspidodasycarpine (4a, $9.2 \mathrm{mg}, 0.0175 \mathrm{mmol})$ was added $\mathrm{Na}$ naphthalenide ${ }^{6}(200 \mu \mathrm{L}$, ca. $0.50 \mathrm{M}$ in THF, ca. $0.10 \mathrm{mmol})$ at $-78^{\circ} \mathrm{C}$. The reaction mixture was stirred at that temperature for $5 \mathrm{~min}$ before it was quenched with saturated aq. $\mathrm{NaHCO}_{3}(5$ $\mathrm{mL})$. The mixture so obtained was extracted with $\mathrm{CHCl}_{3}(5 \times 10 \mathrm{~mL})$, and the combined organic phases were dried over anhydrous $\mathrm{Na}_{2} \mathrm{SO}_{4}$ and filtered. The solvent was evaporated under vacuum, and the residue was subjected to HPLC (Waters 2535Q, SunFire Prep C18 OBD column) using $\mathrm{Et}_{2} \mathrm{NH} / \mathrm{MeCN} /$ water $(3: 700: 300,5.0 \mathrm{~mL} / \mathrm{min})$ as eluent to give aspidodasycarpine $(4,4.9 \mathrm{mg}, 76 \%)$ as a pale yellow oil. 4: $[\alpha]_{\mathrm{D}}^{26}=-79.8\left(\mathrm{c}=0.31\right.$ in $\left.\mathrm{CHCl}_{3}\right) ;^{7} \mathrm{IR}\left(\right.$ film): $v_{\max }=3358,3304,2948,2875,1739$, $1715,1607,1596,1470,1296,1231,1049,910,732 \mathrm{~cm}^{-1} ;{ }^{1} \mathrm{H}$ NMR $\left(400 \mathrm{MHz}, \mathrm{CDCl}_{3}\right): \delta=7.40(\mathrm{~d}, J=$ $7.5 \mathrm{~Hz}, 1 \mathrm{H}), 7.09$ (td, $J=7.7,1.0 \mathrm{~Hz}, 1 \mathrm{H}), 6.79(\mathrm{t}, J=7.4 \mathrm{~Hz}, 1 \mathrm{H}), 6.61(\mathrm{~d}, J=7.8 \mathrm{~Hz}, 1 \mathrm{H}), 5.54(\mathrm{q}$, $J=6.9 \mathrm{~Hz}, 1 \mathrm{H}), 4.30(\mathrm{~s}, 1 \mathrm{H}), 4.06(\mathrm{t}, J=8.1 \mathrm{~Hz}, 1 \mathrm{H}), 3.77(\mathrm{~s}, 3 \mathrm{H}), 3.81-3.72(\mathrm{~m}, 1 \mathrm{H}), 3.62(\mathrm{~d}, J=$ $11.6 \mathrm{~Hz}, 1 \mathrm{H}), 3.59(\mathrm{dd}, J=3.0,3.0 \mathrm{~Hz}, 1 \mathrm{H}), 3.50(\mathrm{ddd}, J=11.3,8.4,5.9 \mathrm{~Hz}, 1 \mathrm{H}), 3.29(\mathrm{~d}, J=13.9 \mathrm{~Hz}$, $1 \mathrm{H}), 3.22(\mathrm{~d}, J=11.5 \mathrm{~Hz}, 1 \mathrm{H}), 3.17$ (dd, $J=2.5,2.5 \mathrm{~Hz}, 1 \mathrm{H}), 2.92-2.79(\mathrm{~m}, 1 \mathrm{H}), 2.64(\mathrm{dd}, J=13.1$, $5.7 \mathrm{~Hz}, 1 \mathrm{H}), 2.09(\mathrm{~d}, J=13.6 \mathrm{~Hz}, 1 \mathrm{H}), 1.78(\mathrm{dt}, J=13.8,3.4 \mathrm{~Hz} 1 \mathrm{H}), 1.69(\mathrm{dd}, J=6.9,1.9 \mathrm{~Hz}, 3 \mathrm{H})$ ppm; ${ }^{13} \mathrm{C}$ NMR $\left(101 \mathrm{MHz}, \mathrm{CDCl}_{3}\right): \delta=174.27,148.44,134.40,130.89,128.88,126.14,123.73,119.57$, 
$109.50,104.36,68.58,67.47,57.86,56.19,53.23,51.80,49.40,39.15,32.03,27.66,13.34$ ppm; HRMS $(m / z):[\mathrm{M}+\mathrm{H}]^{+}$calcd for $\mathrm{C}_{21} \mathrm{H}_{27} \mathrm{~N}_{2} \mathrm{O}_{4}{ }^{+} 371.1965$, found 371.1970 .

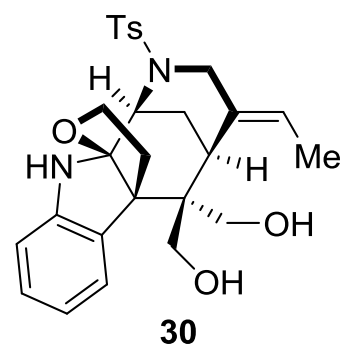

Diol 30: To a stirred solution of pentacyclic alcohol 28 (29.6 mg, $0.0504 \mathrm{mmol})$ in $\mathrm{CH}_{2} \mathrm{Cl}_{2}(2.0 \mathrm{~mL})$ was added $\mathrm{BBr}_{3}\left(400 \mu \mathrm{L}, 1.0 \mathrm{M}\right.$ in $\left.\mathrm{CH}_{2} \mathrm{Cl}_{2}, 0.400 \mathrm{mmol}\right)$ at $-78{ }^{\circ} \mathrm{C}$. The resultant mixture was stirred at that temperature for $1 \mathrm{~h}$ before it was quenched with saturated aq. $\mathrm{NaHCO}_{3}(10 \mathrm{~mL})$ and extracted with $\mathrm{CH}_{2} \mathrm{Cl}_{2}(3 \times 25 \mathrm{~mL})$. The combined organic phases were washed with brine $(10 \mathrm{~mL})$, dried over anhydrous $\mathrm{Na}_{2} \mathrm{SO}_{4}$, and filtered. The volatile was removed under vacuum, and the residue was purified by flash column chromatography with EtOAc/petroleum ether $(1: 2 \rightarrow 1: 1)$ to give diol $\mathbf{3 0}(16.2 \mathrm{mg}$, $65 \%)$ as a colorless oil. 30: $R_{\mathrm{f}}=0.10$ (silica, EtOAc:petroleum ether $\left.1: 2\right) ;[\alpha]_{\mathrm{D}}^{27}=-53.3(c=0.50$ in $\mathrm{CHCl}_{3}$ ); IR (film): $v_{\max }=3508,3356,2930,2878,1733,1596,1485,1468,1318,1254,1156,1099$, 1064, 816, 749, 661, $565 \mathrm{~cm}^{-1} ;{ }^{1} \mathrm{H}$ NMR $\left(400 \mathrm{MHz}, \mathrm{CDCl}_{3}\right): \delta=7.70(\mathrm{~d}, J=8.3 \mathrm{~Hz}, 2 \mathrm{H}), 7.30-7.22$ (m, $3 \mathrm{H}), 7.05(\mathrm{ddd}, J=7.7,7.7,1.1 \mathrm{~Hz}, 1 \mathrm{H}), 6.73(\mathrm{ddd}, J=7.5,7.5,0.9 \mathrm{~Hz}, 1 \mathrm{H}), 6.60(\mathrm{~d}, J=7.6 \mathrm{~Hz}$, $1 \mathrm{H}), 5.55(\mathrm{q}, J=6.9 \mathrm{~Hz}, 1 \mathrm{H}), 4.49(\mathrm{~s}, 1 \mathrm{H}), 4.43(\mathrm{dd}, J=3.2,3.2 \mathrm{~Hz}, 1 \mathrm{H}), 4.00-3.77(\mathrm{~m}, 4 \mathrm{H})$, 3.57-3.51 (m, 2 H), 3.34-3.15 (m, 2 H), 3.07 (d, $J=11.6 \mathrm{~Hz}, 1 \mathrm{H}), 2.48$ (ddd, $J=11.9,11.5,8.1 \mathrm{~Hz}, 1$ H), $2.39(\mathrm{~s}, 3 \mathrm{H}), 2.28-2.13(\mathrm{~m}, 2 \mathrm{H}), 1.68(\mathrm{dd}, J=6.9,1.6 \mathrm{~Hz}, 3 \mathrm{H}), 1.58(\mathrm{ddd}, J=13.9,3.6,3.6 \mathrm{~Hz}, 1$ H) ppm; ${ }^{13} \mathrm{C}$ NMR $\left(101 \mathrm{MHz}, \mathrm{CDCl}_{3}\right): \delta=148.53,142.77,137.68,131.14,130.66,129.29,128.57$, $127.69,125.91,125.58,119.48,109.81,102.84,67.91,65.54,65.17,56.99,53.61,48.58,46.96,35.29$, 29.59, 27.03, 21.62, 13.55 ppm; HRMS $(m / z):[\mathrm{M}+\mathrm{Na}]^{+}$calcd for $\mathrm{C}_{27} \mathrm{H}_{32} \mathrm{~N}_{2} \mathrm{O}_{5} \mathrm{SNa}^{+} 519.1924$, found 519.1922. 


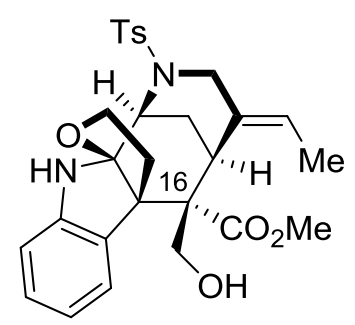

5a: N4-Ts-Ionicerine

N4-Ts-lonicerine (5a): To a stirred solution of diol 30 (16.0 mg, $0.0322 \mathrm{mmol})$ in $\mathrm{CH}_{2} \mathrm{Cl}_{2}(2.0 \mathrm{~mL})$ was added TPAP $(17.0 \mathrm{mg}, 0.0483 \mathrm{mmol})$ at $-30^{\circ} \mathrm{C}$. The reaction mixture was stirred at that temperature for $1 \mathrm{~h}$ before it was directly subjected to flash column chromatography for purification using EtOAc/petroleum ether $(1: 5 \rightarrow 1: 2)$ as eluent to give the $\beta$-hydroxyaldehyde (ca. $3: 1 \mathrm{dr}$ at $\mathrm{C} 16)$ as a pale yellow oil. This oil was dissolved in THF $(500 \mu \mathrm{L})$. To the stirred solution were sequentially added 2-methyl-2-butene (300 $\mu \mathrm{L}), t$ - $\mathrm{BuOH}(100 \mu \mathrm{L})$, aq. $\mathrm{NaH}_{2} \mathrm{PO}_{4}(20.9 \mathrm{mg}$ in $250 \mu \mathrm{L}$ of water), and aq. $\mathrm{NaClO}_{2}\left(7.9 \mathrm{mg}\right.$ in $250 \mu \mathrm{L}$ of water) at $22{ }^{\circ} \mathrm{C}$. The reaction mixture was allowed to stir at that temperature for $24 \mathrm{~h}$ before it was quenched with saturated aq. $\mathrm{NaHSO}_{3}(5 \mathrm{~mL})$. The resultant mixture was extracted with $\mathrm{CHCl}_{3}(4 \times 10 \mathrm{~mL})$, and the combined organic phases were washed with brine $(10$ $\mathrm{mL}$ ), dried over anhydrous $\mathrm{Na}_{2} \mathrm{SO}_{4}$, and filtered. The solvent was evaporated under vacuum, and the residue was dissolved in $\mathrm{MeOH}(1.0 \mathrm{~mL})$. To the stirred solution was added $\mathrm{TMSCHN}_{2} \quad(100 \mu \mathrm{L}, 2.0$ $\mathrm{M}$ in hexanes, $0.200 \mathrm{mmol}$ ) at $0{ }^{\circ} \mathrm{C}$. The mixture so obtained was stirred at that temperature for $10 \mathrm{~min}$ before it was quenched with $\mathrm{AcOH}(30 \mu \mathrm{L})$. The volatile was removed under vacuum, and the residue was purified by flash column chromatography with EtOAc/petroleum ether $(1: 2 \rightarrow 1: 1)$ to give N4-Ts-lonicerine (5a, $11.0 \mathrm{mg}, 65 \%$ for the three steps) and N4-Ts-aspidodasycapine (4a, $2.0 \mathrm{mg}, 11 \%$ for the three steps) both as white foams. 5a: $R_{\mathrm{f}}=0.46$ (silica, EtOAc:petroleum ether 1:2); $[\alpha]_{\mathrm{D}}^{24}=-53.3$ $\left(c=0.22\right.$ in $\left.\mathrm{CHCl}_{3}\right)$; IR (film): $v_{\max }=3539,3358,2926,2872,1705,1608,1486,1469,1324,1260$, $1158,1102,1036,813,750,700,656 \mathrm{~cm}^{-1} ;{ }^{1} \mathrm{H}$ NMR $\left(400 \mathrm{MHz}, \mathrm{CDCl}_{3}\right): \delta=7.70(\mathrm{~d}, J=8.3 \mathrm{~Hz}, 2 \mathrm{H})$, $7.25(\mathrm{~d}, J=8.3 \mathrm{~Hz}, 2 \mathrm{H}), 7.04(\mathrm{~d}, J=7.4 \mathrm{~Hz}, 1 \mathrm{H}), 7.00(\mathrm{ddd}, J=7.7,7.7,1.1 \mathrm{~Hz}, 1 \mathrm{H}), 6.69$ (ddd, $J=$ 7.5, 7.5, 1.1 Hz, $1 \mathrm{H}), 6.55(\mathrm{~d}, J=7.8 \mathrm{~Hz}, 1 \mathrm{H}), 5.52(\mathrm{q}, J=6.8 \mathrm{~Hz}, 1 \mathrm{H}), 4.49(\mathrm{dd}, J=3.3,3.3 \mathrm{~Hz}, 1 \mathrm{H})$, $4.42(\mathrm{~s}, 1 \mathrm{H}), 3.98(\mathrm{~d}, J=14.6 \mathrm{~Hz}, 1 \mathrm{H}), 3.83(\mathrm{dd}, J=12.5,5.1 \mathrm{~Hz}, 1 \mathrm{H}), 3.75(\mathrm{dd}, J=11.3,11.3 \mathrm{~Hz}, 1$ H), $3.68(\mathrm{~d}, J=14.8 \mathrm{~Hz}, 1 \mathrm{H}), 3.47$ (dd, $J=7.6,7.6 \mathrm{~Hz}, 2 \mathrm{H}), 3.21$ (ddd, $J=11.3,8.3,4.8 \mathrm{~Hz}, 1 \mathrm{H})$, 
$3.00(\mathrm{~s}, 3 \mathrm{H}), 2.95$ (ddd, $J=13.9,3.2,3.2 \mathrm{~Hz}, 1 \mathrm{H}), 2.84$ (br s, $1 \mathrm{H}), 2.58-2.47$ (m, $1 \mathrm{H}), 2.46-2.41(\mathrm{~m}$,

$1 \mathrm{H}), 2.40(\mathrm{~s}, 3 \mathrm{H}), 1.59(\mathrm{dd}, J=6.8,1.9 \mathrm{~Hz}, 3 \mathrm{H}), 1.59-1.56(\mathrm{~m}, 1 \mathrm{H}) \mathrm{ppm} ;{ }^{13} \mathrm{C} \mathrm{NMR}(101 \mathrm{MHz}$ $\left.\mathrm{CDCl}_{3}\right): \delta=176.37,148.55,142.71,138.03,133.71,130.55,129.25,128.52,127.65,124.53,124.30$ $119.26,108.19,101.53,66.66,65.42,57.12,55.60,53.53,51.74,48.72,37.06,32.37,28.61,21.63$ 13.36 ppm; $[\mathrm{M}+\mathrm{H}]^{+}$calcd for $\mathrm{C}_{28} \mathrm{H}_{33} \mathrm{~N}_{2} \mathrm{O}_{5} \mathrm{~S}^{+}$525.2054, found 525.2046.

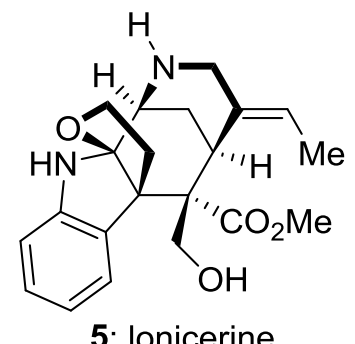

Lonicerine (5): To a stirred solution of N4-Ts-lonicerine (5a, $3.2 \mathrm{mg}, 0.0061 \mathrm{mmol})$ in THF (1.0 mL) was added $\mathrm{Na}$ naphthalenide ${ }^{6}(100 \mu \mathrm{L}$, ca. $0.50 \mathrm{M}$ in THF, ca. $0.050 \mathrm{mmol})$ at $-78{ }^{\circ} \mathrm{C}$. The reaction mixture was stirred at that temperature for $5 \mathrm{~min}$ before it was quenched with saturated aq. $\mathrm{NaHCO}_{3}(2$ $\mathrm{mL})$. The mixture so obtained was extracted with $\mathrm{CHCl}_{3}(5 \times 5 \mathrm{~mL})$, and the combined organic phases were dried over anhydrous $\mathrm{Na}_{2} \mathrm{SO}_{4}$ and filtered. The solvent was evaporated under vacuum, and the residue was subjected to HPLC (Waters 2535Q, SunFire Prep C18 OBD column) using $\mathrm{Et}_{2} \mathrm{NH} / \mathrm{MeCN} /$ water $(3: 700: 300,5.0 \mathrm{~mL} / \mathrm{min})$ as eluent to give lonicerine $(\mathbf{5}, 1.7 \mathrm{mg}, 75 \%)$ as a pale yellow oil. 5: $[\alpha]_{\mathrm{D}}^{21}=-103.9\left(c=0.080\right.$ in $\left.\mathrm{CHCl}_{3}\right){ }^{7}$ IR (film): $v_{\max }=3539,3330,2961,2925,2856$, 1706, 1608, 1485, 1468, 1262, 1224, 1080, 1033, 801, $751 \mathrm{~cm}^{-1} ;{ }^{1} \mathrm{H}$ NMR $\left(500 \mathrm{MHz}, \mathrm{CDCl}_{3}\right): \delta=7.07$ $(\mathrm{d}, J=7.5 \mathrm{~Hz}, 1 \mathrm{H}), 7.00(\mathrm{td}, J=7.6,0.9 \mathrm{~Hz}, 1 \mathrm{H}), 6.69(\mathrm{t}, J=7.5 \mathrm{~Hz}, 1 \mathrm{H}), 6.54(\mathrm{~d}, J=7.7 \mathrm{~Hz}, 1 \mathrm{H})$ $5.55(\mathrm{q}, J=6.8 \mathrm{~Hz}, 1 \mathrm{H}), 4.36($ br s, $1 \mathrm{H}), 4.06(\mathrm{dd}, J=8.5,7.3 \mathrm{~Hz}, 1 \mathrm{H}), 3.87(\mathrm{~d}, J=12.5 \mathrm{~Hz}, 1 \mathrm{H})$, $3.78(\mathrm{~d}, J=12.5 \mathrm{~Hz}, 1 \mathrm{H}), 3.59(\mathrm{dt}, J=13.4,2.0 \mathrm{~Hz}, 1 \mathrm{H}), 3.47-3.39(\mathrm{~m}, 1 \mathrm{H}), 3.37$ (s, $1 \mathrm{H}), 3.30(\mathrm{~d}, J$ $=13.5 \mathrm{~Hz}, 1 \mathrm{H}), 3.01(\mathrm{~s}, 3 \mathrm{H}), 2.92-2.76(\mathrm{~m}, 3 \mathrm{H}), 2.53(\mathrm{dd}, J=12.6,4.3 \mathrm{~Hz}, 1 \mathrm{H}), 1.80(\mathrm{dt}, J=13.4$, $3.2 \mathrm{~Hz}, 1 \mathrm{H}), 1.62(\mathrm{dd}, J=6.8,1.8 \mathrm{~Hz}, 3 \mathrm{H}) \mathrm{ppm} ;{ }^{13} \mathrm{C} \mathrm{NMR}\left(126 \mathrm{MHz}, \mathrm{CDCl}_{3}\right): \delta=176.96,148.66$, $134.54,133.76,128.28,124.55,122.00,118.97,107.54,102.57,66.78,65.40,57.58,55.31,53.35$ 51.61, 48.80, 37.54, 33.58, 28.30, 13.13 ppm; HRMS $(m / z):[\mathrm{M}+\mathrm{H}]^{+}$calcd for $\mathrm{C}_{21} \mathrm{H}_{27} \mathrm{~N}_{2} \mathrm{O}_{4}{ }^{+} 371.1965$, found 371.1970 . 


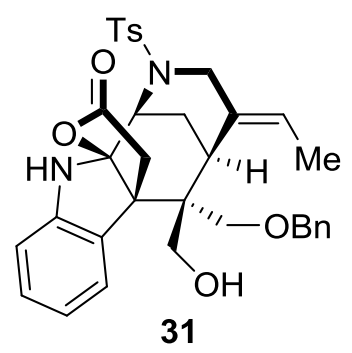

Pentacyclic alcohol 31: To a stirred solution of lactol $27(67.0 \mathrm{mg}, 0.115 \mathrm{mmol})$ in $\mathrm{CH}_{2} \mathrm{Cl}_{2}(5.0 \mathrm{~mL})$ were sequentially added NMO $(20.2 \mathrm{mg}, 0.172 \mathrm{mmol})$ and TPAP $(8.0 \mathrm{mg}, 0.023 \mathrm{mmol})$ at $0{ }^{\circ} \mathrm{C}$. The reaction mixture was stirred at that temperature for $30 \mathrm{~min}$ and then directly passed through a plug of silica using EtOAc/petroleum ether $(1: 2 \rightarrow 2: 1)$ as eluent to give the lactone $(57.6 \mathrm{mg}, 86 \%)$ as a pale yellow oil. This oil was dissolved in THF $(5.0 \mathrm{~mL})$. To the stirred solution was added aq. $\mathrm{LiOH}(5.0 \mathrm{~mL}$, 1.0 M). The resultant mixture was allowed to stir at that temperature for $12 \mathrm{~h}$ before it was quenched with saturated aq. $\mathrm{NaHCO}_{3}(10 \mathrm{~mL})$ and extracted with EtOAc $(3 \times 15 \mathrm{~mL})$. The combined organic phases were washed with brine $(10 \mathrm{~mL})$, dried over anhydrous $\mathrm{Na}_{2} \mathrm{SO}_{4}$, filtered, and concentrated under vacuum. The residue was subjected to flash column chromatography for purification using EtOAc/petroleum ether $(1: 4 \rightarrow 2: 1)$ as eluent to give pentacyclic alcohol $\mathbf{3 1}(56.1 \mathrm{mg}, 94 \%)$ as a white powder. 31: $R_{\mathrm{f}}=0.30$ (silica, EtOAc:petroleum ether 2:3); $[\alpha]_{\mathrm{D}}^{25}=-43.9\left(c=0.10\right.$ in $\left.\mathrm{CHCl}_{3}\right)$; IR (film): $v_{\max }=3357,2963,2926,2852,1766,1596,1473,1331,1260,1160,1097,879,665,548 \mathrm{~cm}^{-1} ;{ }^{1} \mathrm{H}$ NMR $\left(400 \mathrm{MHz}, \mathrm{CDCl}_{3}\right): \delta=7.73(\mathrm{~d}, J=8.3 \mathrm{~Hz}, 2 \mathrm{H}), 7.36(\mathrm{~d}, J=7.6 \mathrm{~Hz}, 1 \mathrm{H}), 7.32-7.24(\mathrm{~m}, 5 \mathrm{H})$, 7.14-7.05 (m, $3 \mathrm{H}), 6.79(\mathrm{ddd}, J=7.6,7.6,0.7 \mathrm{~Hz}, 1 \mathrm{H}), 6.64(\mathrm{~d}, J=7.7 \mathrm{~Hz}, 1 \mathrm{H}), 5.57$ (q, $J=6.8 \mathrm{~Hz}, 1$ H), $5.02(\mathrm{~s}, 1 \mathrm{H}), 4.69(\mathrm{dd}, J=3.2,3.2 \mathrm{~Hz}, 1 \mathrm{H}), 4.17-4.01(\mathrm{~m}, 3 \mathrm{H}), 3.84(\mathrm{dd}, J=11.3,4.8 \mathrm{~Hz}, 1 \mathrm{H})$, $3.74(\mathrm{~d}, J=16.1 \mathrm{~Hz}, 1 \mathrm{H}), 3.65(\mathrm{dd}, J=11.3,5.3 \mathrm{~Hz}, 1 \mathrm{H}), 3.26(\mathrm{~s}, 1 \mathrm{H}), 3.23(\mathrm{~d}, J=18.4 \mathrm{~Hz}, 1 \mathrm{H})$, $3.15(\mathrm{~d}, J=9.8 \mathrm{~Hz}, 1 \mathrm{H}), 3.07(\mathrm{~d}, J=18.4 \mathrm{~Hz}, 1 \mathrm{H}), 2.96(\mathrm{~d}, J=9.8 \mathrm{~Hz}, 1 \mathrm{H}), 2.40(\mathrm{~s}, 3 \mathrm{H}), 2.27-2.15$ (m, $1 \mathrm{H}), 2.04(\mathrm{dd}, J=5.2,5.2 \mathrm{~Hz}, 1 \mathrm{H}), 1.63(\mathrm{dd}, J=6.8,1.5 \mathrm{~Hz}, 3 \mathrm{H}), 1.57$ (ddd, $J=10.4,3.6,3.6 \mathrm{~Hz}$, $1 \mathrm{H}) \mathrm{ppm} ;{ }^{13} \mathrm{C} \mathrm{NMR}\left(101 \mathrm{MHz}, \mathrm{CDCl}_{3}\right): \delta=173.44,146.29,143.51,138.00,136.74,131.13,129.77$, $129.63,129.10,128.45,127.79,127.73,127.66,127.56,125.88,120.55,110.44,105.30,73.11,70.75$, 64.40, 53.59, 51.58, 48.01, 46.97, 39.88, 29.33, 26.28, 21.64, 13.65 ppm; HRMS $(m / z):[M+H]^{+}$calcd for $\mathrm{C}_{34} \mathrm{H}_{37} \mathrm{~N}_{2} \mathrm{O}_{6}{ }^{+}$601.2367, found 601.2355 . 


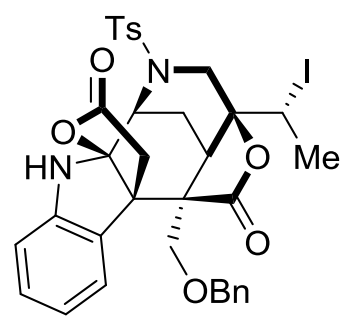

33

Iodolactone 33: To a stirred solution of pentacyclic alcohol $31(51.5 \mathrm{mg}, 0.0857 \mathrm{mmol})$ in $\mathrm{CH}_{2} \mathrm{Cl}_{2} /$ methyl-2-butene $(5.2 \mathrm{~mL}, 3: 1)$ were sequentially added aq. $\mathrm{AcOH} / \mathrm{NaOAc}$ buffer $(1.0 \mathrm{M}, \mathrm{pH}=$ 4, $4.0 \mathrm{~mL})$, AZADO (15.6 mg, $0.0947 \mathrm{mmol})$, and $\mathrm{NaClO}_{2}(77.4 \mathrm{mg}, 0.860 \mathrm{mmol})$ at $22{ }^{\circ} \mathrm{C} .{ }^{4} \mathrm{The}$ reaction mixture was stirred at that temperature for $10 \mathrm{~h}$ before it was quenched with saturated aq. $\mathrm{NaHSO}_{3}(20 \mathrm{~mL})$ and extracted with $\mathrm{CHCl}_{3}(4 \times 20 \mathrm{~mL})$. The combined organic phases were washed with brine $(10 \mathrm{~mL})$, dried over anhydrous $\mathrm{Na}_{2} \mathrm{SO}_{4}$, and filtered. The volatile was evaporated under vacuum, and the residue (crude carboxylic acid) was dissolved in $\mathrm{MeCN}(6.0 \mathrm{~mL})$. To the stirred solution was added $\mathrm{NaHCO}_{3}(20.7 \mathrm{mg}, 0.247 \mathrm{mmol})$ and $\mathrm{I}_{2}(62.0 \mathrm{mg}, 0.247 \mathrm{mmol})$ at $0{ }^{\circ} \mathrm{C}$. The resultant mixture was stirred at that temperature for $30 \mathrm{~min}$ before it was quenched with saturated aq. $\mathrm{NaHSO}_{3}$ solution $(10 \mathrm{~mL})$. The mixture so obtained was extracted with EtOAc $(3 \times 20 \mathrm{~mL})$. The combined organic phases were washed with brine $(15 \mathrm{~mL})$, dried over anhydrous $\mathrm{Na}_{2} \mathrm{SO}_{4}$, filtered, and concentrated under vacuum. The residue was purified by flash column chromatography with EtOAc/petroleum ether $(1: 2 \rightarrow 1: 1)$ to give iodolactone $\mathbf{3 3}(58.0 \mathrm{mg}, 91 \%$ for the two steps) as a pale yellow powder. 32: $R_{\mathrm{f}}=0.43$ (silica, EtOAc:petroleum ether 1:2); $[\alpha]_{\mathrm{D}}^{27}=-26.2\left(c=0.50\right.$ in $\left.\mathrm{CHCl}_{3}\right)$; IR (film): $v_{\max }=3365,2925,2860,1777,1611,1597,1473,1452,1356,1214,1165,1091,752,664 \mathrm{~cm}^{-1}$; ${ }^{1} \mathrm{H}$ NMR (400 MHz, $\left.\mathrm{CDCl}_{3}\right): \delta=7.83(\mathrm{~d}, J=8.2 \mathrm{~Hz}, 2 \mathrm{H}), 7.33(\mathrm{~d}, J=8.1 \mathrm{~Hz}, 2 \mathrm{H}), 7.30-7.21(\mathrm{~m}, 3 \mathrm{H})$, $7.18(\mathrm{~d}, J=7.2 \mathrm{~Hz}, 1 \mathrm{H}), 7.12-7.06(\mathrm{~m}, 2 \mathrm{H}), 7.03(\mathrm{dd}, J=7.4,7.4 \mathrm{~Hz}, 1 \mathrm{H}), 6.81(\mathrm{dd}, J=7.5,7.5 \mathrm{~Hz}, 1$ H), $6.60(\mathrm{~d}, J=7.8 \mathrm{~Hz}, 1 \mathrm{H}), 5.70(\mathrm{br} \mathrm{s}, 1 \mathrm{H}), 4.90(\mathrm{~d}, J=3.2 \mathrm{~Hz}, 1 \mathrm{H}), 4.19(\mathrm{~d}, J=11.5 \mathrm{~Hz}, 1 \mathrm{H}), 4.10$ $(\mathrm{d}, J=11.5 \mathrm{~Hz}, 1 \mathrm{H}), 4.08(\mathrm{~d}, J=13.7 \mathrm{~Hz}, 1 \mathrm{H}), 3.61(\mathrm{~d}, J=14.0 \mathrm{~Hz}, 1 \mathrm{H}), 3.58(\mathrm{~d}, J=9.5 \mathrm{~Hz}, 1 \mathrm{H})$, $3.33(\mathrm{~d}, J=13.7 \mathrm{~Hz}, 1 \mathrm{H}), 3.11-2.96(\mathrm{~m}, 3 \mathrm{H}), 2.80(\mathrm{~d}, J=9.5 \mathrm{~Hz}, 1 \mathrm{H}), 2.53(\mathrm{dd}, J=14.6,2.9 \mathrm{~Hz}, 1 \mathrm{H})$, $2.44(\mathrm{~s}, 3 \mathrm{H}), 2.01(\mathrm{~d}, J=14.6 \mathrm{~Hz}, 1 \mathrm{H}), 1.76(\mathrm{~d}, J=7.0 \mathrm{~Hz}, 3 \mathrm{H}) \mathrm{ppm} ;{ }^{13} \mathrm{C} \mathrm{NMR}\left(101 \mathrm{MHz}, \mathrm{CDCl}_{3}\right): \delta$ $=175.05,171.86,147.05,144.94,137.15,133.34,130.19,130.13,128.50,128.34,127.82,127.69$, 
$127.55,126.09,120.08,111.37,104.86,83.38,73.49,71.18,53.26,51.17,50.09,49.27,43.00,34.43$,

31.59, 23.24, 22.50, 21.69 ppm; HRMS $(m / z):[M+H]^{+}$calcd for $\mathrm{C}_{34} \mathrm{H}_{34} \mathrm{IN}_{2} \mathrm{O}_{7} \mathrm{~S}^{+} 741.1126$, found 741.1128.

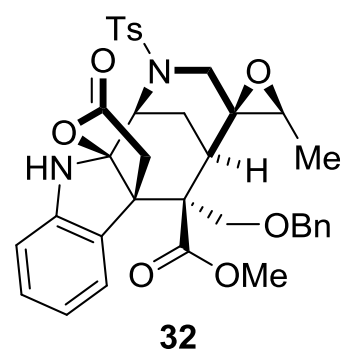

Epoxide 32: To a stirred solution of iodolactone $33(58.0 \mathrm{mg}, 0.0783 \mathrm{mmol})$ in $\mathrm{MeOH}(5.0 \mathrm{~mL})$ was added $\mathrm{Na}_{2} \mathrm{CO}_{3}(83.0 \mathrm{mg}, 0.783 \mathrm{mmol})$ at $22{ }^{\circ} \mathrm{C}$. The resultant mixture was stirred at that temperature for $2 \mathrm{~d}$ before it was quenched with saturated aq. $\mathrm{NaHCO}_{3}(15 \mathrm{~mL})$. The mixture so obtained was extracted with EtOAc $(3 \times 20 \mathrm{~mL})$, and the combined organic phases were washed with brine $(15 \mathrm{~mL})$, dried over anhydrous $\mathrm{Na}_{2} \mathrm{SO}_{4}$, and filtered. The solvent was evaporated under vacuum, and the residue was subjected to flash column chromatography for purification using EtOAc/petroleum ether $(1: 5 \rightarrow 1: 3)$ as eluent to give epoxide $32(39.7 \mathrm{mg}, 79 \%)$ as a white powder. $R_{\mathrm{f}}=0.41$ (silica, EtOAc:petroleum ether 1:2); m.p. $[( \pm)-32]: 122-125{ }^{\circ} \mathrm{C}$ (EtOAc:hexane 1:1); $[\alpha]_{\mathrm{D}}^{24}=-87.2\left(c=1.0\right.$ in $\left.\mathrm{CHCl}_{3}\right)$; IR (film): $v_{\max }=$ 3348, 2961, 1773, 1743, 1736, 1468, 1259, 1167, 1093, 1026, 817, 809, 667, $571 \mathrm{~cm}^{-1} ;{ }^{1} \mathrm{H}$ NMR (400 $\left.\mathrm{MHz}, \mathrm{CDCl}_{3}\right): \delta=7.74(\mathrm{~d}, J=8.3 \mathrm{~Hz}, 2 \mathrm{H}), 7.47(\mathrm{~d}, J=7.5 \mathrm{~Hz}, 1 \mathrm{H}), 7.28(\mathrm{~d}, J=8.1 \mathrm{~Hz}, 2 \mathrm{H})$, 7.24-7.16 (m, $3 \mathrm{H}), 7.06(\mathrm{ddd}, J=7.7,7.7,1.1 \mathrm{~Hz}, 1 \mathrm{H}), 7.00-6.92(\mathrm{~m}, 2 \mathrm{H}), 6.76(\mathrm{dd}, J=7.2,7.2 \mathrm{~Hz}$, $1 \mathrm{H}), 6.67(\mathrm{~d}, J=7.7 \mathrm{~Hz}, 1 \mathrm{H}), 5.04(\mathrm{~s}, 1 \mathrm{H}), 4.71(\mathrm{dd}, J=3.2,3.2 \mathrm{~Hz}, 1 \mathrm{H}), 3.66(\mathrm{~d}, J=19.1 \mathrm{~Hz}, 1 \mathrm{H})$, 3.67-3.59 (m, $2 \mathrm{H}), 3.62(\mathrm{~s}, 3 \mathrm{H}), 3.48(\mathrm{~d}, J=19.1 \mathrm{~Hz}, 1 \mathrm{H}), 3.45(\mathrm{~d}, J=14.1 \mathrm{~Hz}, 1 \mathrm{H}), 3.25(\mathrm{~d}, J=9.3$ $\mathrm{Hz}, 1 \mathrm{H}), 3.03(\mathrm{~d}, J=9.3 \mathrm{~Hz}, 1 \mathrm{H}), 2.89(\mathrm{q}, J=5.5 \mathrm{~Hz}, 1 \mathrm{H}), 2.88(\mathrm{~d}, J=14.1 \mathrm{~Hz}, 1 \mathrm{H}), 2.76(\mathrm{dd}, J=$ 3.1, 3.1 Hz, $1 \mathrm{H}), 2.40$ (s, $3 \mathrm{H}), 2.17$ (ddd, $J=15.0,3.0,3.0 \mathrm{~Hz}, 1 \mathrm{H}), 1.82$ (ddd, $J=15.0,3.6,3.6 \mathrm{~Hz}, 1$ $\mathrm{H}), 1.38(\mathrm{~d}, J=5.5 \mathrm{~Hz}, 3 \mathrm{H}) \mathrm{ppm} ;{ }^{13} \mathrm{C} \mathrm{NMR}\left(101 \mathrm{MHz}, \mathrm{CDCl}_{3}\right): \delta=174.10,171.95,146.61,144.32$, $138.03,134.32,131.06,129.94,129.18,128.45,128.15,128.13,127.32,127.08,120.96,110.87,104.57$, $74.12,72.58,62.34,58.61,54.69,52.90,51.38,50.30,48.67,41.17,31.18,24.08,21.69,11.18$ ppm; HRMS $(m / z):[\mathrm{M}+\mathrm{H}]^{+}$calcd for $\mathrm{C}_{35} \mathrm{H}_{37} \mathrm{~N}_{2} \mathrm{O}_{8} \mathrm{~S}^{+}$645.2271, found 645.2259. CCDC 1052434 contains 
the supplementary crystallographic data of $( \pm)-32$. These data can be obtained free of charge from The Cambridge Crystallographic Data Centre via www.ccdc.cam.ac.uk/data_request/cif.

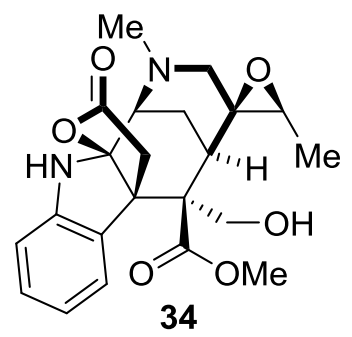

Epoxyalcohol 34: To a stirred solution of epoxide $32(14.2 \mathrm{mg}, 0.0220 \mathrm{mmol})$ in THF $(2.0 \mathrm{~mL})$ was added $\mathrm{Na}$ naphthalenide ${ }^{6}(500 \mu \mathrm{L}$, ca. $0.50 \mathrm{M}$ in THF, ca. $0.25 \mathrm{mmol})$ at $-78{ }^{\circ} \mathrm{C}$. The reaction mixture was stirred at that temperature for $5 \mathrm{~min}$ before it was quenched with saturated aq. $\mathrm{NaHCO}_{3}(5 \mathrm{~mL})$. The mixture so obtained was extracted with $\mathrm{CHCl}_{3}(5 \times 15 \mathrm{~mL})$, and the combined organic phases were dried over anhydrous $\mathrm{Na}_{2} \mathrm{SO}_{4}$ and filtered. The solvent was removed under vacuum, and the residue was dissolved in $\mathrm{MeOH}(2.0 \mathrm{~mL})$. To the solution were sequentially added aq. HCHO (1.0 mL, $37 \mathrm{wt} \%)$ and $\mathrm{Pd} / \mathrm{C}(12.0 \mathrm{mg}, 10 \mathrm{wt} \%)$ at $22{ }^{\circ} \mathrm{C}$. The resultant mixture was stirred under a hydrogen atmosphere at that temperature for $3 \mathrm{~h}$. After removal of the volatile under vacuum, the residue was directly subjected to HPLC (Waters 2535Q, SunFire Prep C18 OBD column) for purification using $\mathrm{Et}_{2} \mathrm{NH} / \mathrm{MeCN} /$ water (3:700:300, $5.0 \mathrm{~mL} / \mathrm{min})$ as eluent to give epoxyalcohol $34(4.5 \mathrm{mg}, 50 \%$ for the two steps) as a pale yellow oil. 34: $[\alpha]_{\mathrm{D}}^{26}=-24.2\left(c=0.05\right.$ in $\left.\mathrm{CHCl}_{3}\right)$; IR (film): $v_{\max }=3566,3348,2962,2925,2853,1739$, 1610, 1486, 1261, 1092, 1020, 801, 696, $668 \mathrm{~cm}^{-1} ;{ }^{1} \mathrm{H}$ NMR $\left(500 \mathrm{MHz}, \mathrm{CDCl}_{3}\right): \delta=7.40(\mathrm{~d}, J=7.6 \mathrm{~Hz}$, $1 \mathrm{H}), 7.16(\mathrm{dd}, J=7.7,7.7 \mathrm{~Hz}, 1 \mathrm{H}), 6.89(\mathrm{dd}, J=7.5,7.5 \mathrm{~Hz}, 1 \mathrm{H}), 6.76(\mathrm{~d}, J=7.8 \mathrm{~Hz}, 1 \mathrm{H}), 4.67(\mathrm{~s}, 1$ H), $3.80(\mathrm{~d}, J=18.5 \mathrm{~Hz}, 1 \mathrm{H}), 3.72(\mathrm{~s}, 3 \mathrm{H}), 3.50(\mathrm{~d}, J=18.5 \mathrm{~Hz}, 1 \mathrm{H}), 3.42(\mathrm{dd}, J=12.1,12.1 \mathrm{~Hz}, 1 \mathrm{H})$, $3.21(\mathrm{~d}, J=13.4 \mathrm{~Hz}, 1 \mathrm{H}), 3.17(\mathrm{~d}, J=11.7 \mathrm{~Hz}, 1 \mathrm{H}), 3.00(\mathrm{br} \mathrm{s}, 1 \mathrm{H}), 2.86-2.81(\mathrm{~m}, 2 \mathrm{H}), 2.40$ (s, $3 \mathrm{H})$, $2.19(\mathrm{~d}, J=13.4 \mathrm{~Hz}, 1 \mathrm{H}), 2.14-2.00(\mathrm{~m}, 2 \mathrm{H}), 1.79(\mathrm{~d}, J=11.0 \mathrm{~Hz}, 1 \mathrm{H}), 1.39(\mathrm{~d}, J=5.5 \mathrm{~Hz}, 3 \mathrm{H}) \mathrm{ppm}$; ${ }^{13} \mathrm{C}$ NMR $\left(126 \mathrm{MHz}, \mathrm{CDCl}_{3}\right): \delta=174.62,174.06,147.89,131.51,129.65,126.60,121.62,111.70$ $106.44,66.27,61.99,59.55,57.47,56.76,54.93,53.51,51.91,45.63,42.40,29.48,21.73,10.96$ ppm; $\operatorname{HRMS}(m / z):[\mathrm{M}+\mathrm{H}]^{+}$calcd for $\mathrm{C}_{22} \mathrm{H}_{27} \mathrm{~N}_{2} \mathrm{O}_{2}{ }^{+} 415.1864$, found 415.1859. 


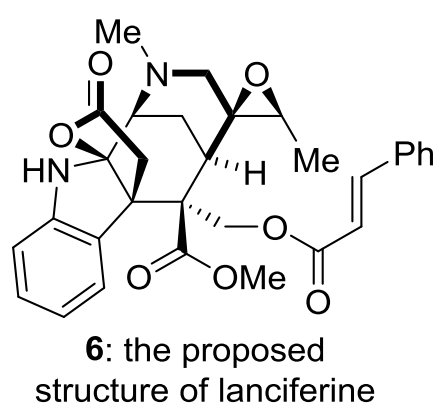

The proposed structure of lanciferine (6): To a stirred solution of epoxyalcohol $\mathbf{3 4}$ (2.1 $\mathrm{mg}, 0.0051$ mmol) in pyridine $(600 \mu \mathrm{L})$ were sequentially added 4-DMAP $(2.0 \mathrm{mg}, 0.016 \mathrm{mmol})$ and cinnamic anhydride $(6.3 \mathrm{mg}, 0.023 \mathrm{mmol})$ at $22{ }^{\circ} \mathrm{C}$. The resultant mixture was warmed to $50{ }^{\circ} \mathrm{C}$ and stirred at that temperature for $2 \mathrm{~h}$ before it was cooled to $22{ }^{\circ} \mathrm{C}$ and quenched with saturated aq. $\mathrm{NaHCO}_{3}(5 \mathrm{~mL})$. The mixture so obtained was extracted with $\mathrm{CHCl}_{3}(5 \times 5 \mathrm{~mL})$. The combined organic phases were washed with brine $(5 \mathrm{~mL})$, dried over anhydrous $\mathrm{Na}_{2} \mathrm{SO}_{4}$, filtered, and concentrated under vacuum. The residue was subjected to HPLC (Waters 2535Q, SunFire Prep C18 OBD column) for purification using $\mathrm{Et}_{2} \mathrm{NH} / \mathrm{MeCN} /$ water $(3: 700: 300,5.0 \mathrm{~mL} / \mathrm{min})$ as eluent to give the proposed structure of lanciferine $(\mathbf{6}$, $2.2 \mathrm{mg}, 79 \%)$ as a white foam. 6: $[\alpha]_{\mathrm{D}}^{23}=-56.2\left(c=0.086\right.$ in $\left.\mathrm{CHCl}_{3}\right)$; IR (film): $v_{\max }=3366,2917$, 2846, 1736, 1466, 1199, 1172, 1091, 1019, 768, $632 \mathrm{~cm}^{-1} ;{ }^{1} \mathrm{H}$ NMR $\left(600 \mathrm{MHz}, \mathrm{CDCl}_{3}\right): \delta=7.58(\mathrm{~d}, J=$ 7.5 Hz, $1 \mathrm{H}$ ), 7.48-7.43 (m, $2 \mathrm{H}), 7.43-7.37$ (m, $3 \mathrm{H}), 7.35$ (d, $J=16.0 \mathrm{~Hz}, 1 \mathrm{H}), 7.03$ (ddd, $J=7.7,7.7$, $1.0 \mathrm{~Hz}, 1 \mathrm{H}), 6.80(\mathrm{dd}, J=7.5,7.5 \mathrm{~Hz}, 1 \mathrm{H}), 6.70(\mathrm{~d}, J=7.8 \mathrm{~Hz}, 1 \mathrm{H}), 6.00(\mathrm{~d}, J=16.0 \mathrm{~Hz}, 1 \mathrm{H}), 4.66(\mathrm{~s}$, $1 \mathrm{H}), 4.27(\mathrm{~d}, J=11.2 \mathrm{~Hz}, 1 \mathrm{H}), 3.92(\mathrm{~d}, J=11.2 \mathrm{~Hz}, 1 \mathrm{H}), 3.77(\mathrm{~d}, J=18.7 \mathrm{~Hz}, 1 \mathrm{H}), 3.64(\mathrm{~d}, J=18.7$ Hz, $1 \mathrm{H}), 3.62(\mathrm{~s}, 3 \mathrm{H}), 3.22(\mathrm{~d}, J=13.5 \mathrm{~Hz}, 1 \mathrm{H}), 3.09-3.01(\mathrm{~m}, 1 \mathrm{H}), 2.84$ (q, $J=5.6 \mathrm{~Hz}, 1 \mathrm{H}), 2.66$ (dd, $J=3.1,3.1 \mathrm{~Hz}, 1 \mathrm{H}), 2.41$ (s, $3 \mathrm{H}), 2.22(\mathrm{~d}, J=13.5 \mathrm{~Hz}, 1 \mathrm{H}), 2.16$ (ddd, $J=14.8,2.5,2.5 \mathrm{~Hz}, 1 \mathrm{H}$ ), $2.12(\mathrm{ddd}, J=14.8,4.0,4.0 \mathrm{~Hz}, 1 \mathrm{H}), 1.41(\mathrm{~d}, J=5.6 \mathrm{~Hz}, 3 \mathrm{H}) \mathrm{ppm} ;{ }^{13} \mathrm{C} \mathrm{NMR}\left(151 \mathrm{MHz}, \mathrm{CDCl}_{3}\right): \delta=$ $174.72,171.44,165.37,147.25,144.86,134.34,131.88,130.56,129.34,129.09,128.20,128.15,121.19$, $117.39,111.29,106.43,68.49,61.93,59.29,57.26,56.65,54.28,53.52,51.54,45.61,41.94,32.65$, 22.24, 10.82 ppm; HRMS (m/z): $[\mathrm{M}+\mathrm{H}]^{+}$calcd for $\mathrm{C}_{31} \mathrm{H}_{33} \mathrm{~N}_{2} \mathrm{O}_{7}{ }^{+}$545.2282, found 545.2277. 


\section{References}

(1) (a) Li, X.; Vince, R. Bioorg. Med. Chem. 2006, 14, 2942. (b) Ergün, Y.; Bayraktar, N.; Patir, S.; Okay, G. J. Heterocycl. Chem. 2000, 37, 11.

(2) Müller, T. E.; Green, J. C.; Mingos, D. M. P.; McPartlin, C. M.; Whittingham, C.; Williams, D. J.; Woodroffe, T. M. J. Organomet. Chem. 1998, 551, 313.

(3) Okada, Y.; Ohtsu, M.; Bando, M.; Yamada, H. Chem. Lett. 2007, 26, 992.

(4) Oltyoort, J. J.; Van Boeckel, C. A. A.; De Koning, J. H.; Van Boom, J. H. Synthesis 1981, 305.

(5) (a) Shibuya, M.; Sato T.; Tomizawa, M.; Iwabuchi, Y. Chem. Commun. 2009, 1739. (b) Deng, J.; Zhou, S.; Zhang, W.; Li, J.; Li, R.; Li, A. J. Am. Chem. Soc. 2014, 136, 8185.

(6) (a) Zhang, M.; Huang, X.; Shen, L.; Qin, Y. J. Am. Chem. Soc. 2009, 131, 6013. (b) Teng, M.; Zi, W.; Ma, D. Angew. Chem., Int. Ed. 2014, 53, 1814.

(7) Prof. Toh-Seok Kam provided the optical rotation direction and values of their isolated samples of naturally occurring aspidodasycarpine (4) and lonicerine (5). 4: $[\alpha]_{\mathrm{D}}=-70\left(c=0.31\right.$ in $\left.\mathrm{CHCl}_{3}\right) ; 5:[\alpha]_{\mathrm{D}}$ $=-127\left(c=0.76\right.$ in $\left.\mathrm{CHCl}_{3}\right)$. 


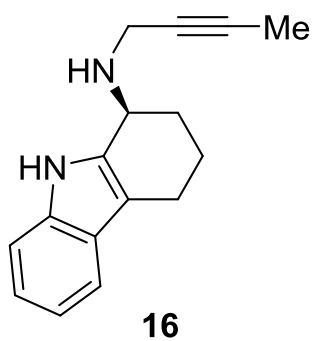

A racemic sample of compound $\mathbf{1 6}$ was obtained through $\mathrm{NaBH}_{4}$ reduction of the corresponding imine. The racemic and optically active $\mathbf{1 6}$ were analyzed with HPLC (CHIRALPAK ID-3 column, $\mathrm{Et}_{2} \mathrm{NH}: i-\mathrm{PrOH}:$ hexane $\left.=1: 500: 500,0.70 \mathrm{~mL} / \mathrm{min}\right)$ to determine the retention time and enantiomeric excesses. For $(-)-\mathbf{1 6}$, ee $=94.8 \%$.
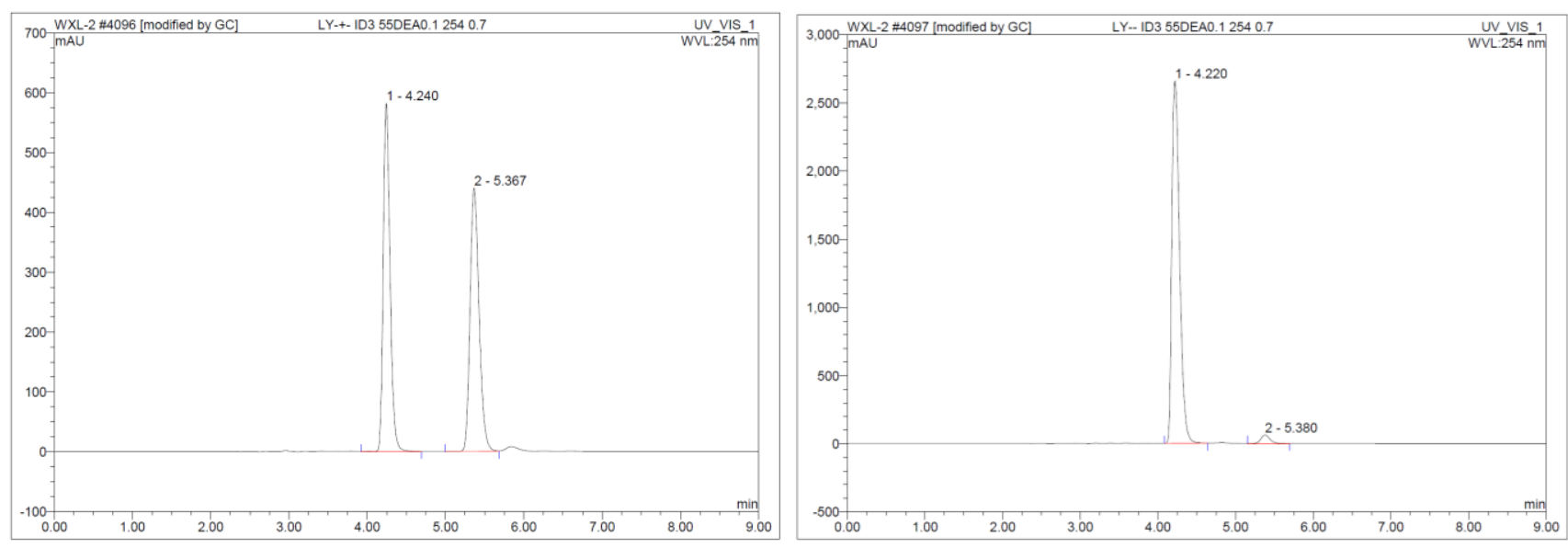

\begin{tabular}{|r|ccrrrrr|}
\hline No. & $\begin{array}{c}\text { Ret.Time } \\
\text { min }\end{array}$ & Peak Name & $\begin{array}{c}\text { Height } \\
\text { mAU }\end{array}$ & $\begin{array}{r}\text { Area } \\
\mathrm{mAU}^{*} \text { min }\end{array}$ & $\begin{array}{r}\text { Rel.Area } \\
\%\end{array}$ & Amount & Type \\
\hline 1 & 4.24 & n.a. & 581.871 & 58.412 & 49.94 & n.a. & BMB $^{*}$ \\
2 & 5.37 & n.a. & 439.579 & 58.554 & 50.06 & n.a. & BMB $^{*}$ \\
\hline Total: & & & 1021.451 & 116.966 & 100.00 & 0.000 & \\
\hline
\end{tabular}

\begin{tabular}{|r|rrrrrrr|}
\hline No. & $\begin{array}{c}\text { Ret.Time } \\
\text { min }\end{array}$ & Peak Name & $\begin{array}{c}\text { Height } \\
\text { mAU }\end{array}$ & $\begin{array}{c}\text { Area } \\
\text { mAU*min }\end{array}$ & $\begin{array}{r}\text { Rel.Area } \\
\%\end{array}$ & Amount & Type \\
\hline 1 & 4.22 & n.a. & 2661.050 & 308.189 & 97.39 & n.a. & BMB $^{*}$ \\
2 & 5.38 & n.a. & 62.684 & 8.254 & 2.61 & n.a. & BMB $^{*}$ \\
\hline Total: & & & 2723.734 & 316.443 & 100.00 & 0.000 & \\
\hline
\end{tabular}




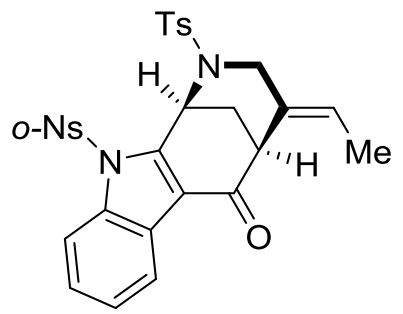

18

The racemic and optically active $\mathbf{1 8}$ were analyzed with HPLC (CHIRALPAK IC column, $\mathrm{Et}_{2} \mathrm{NH}: i-\mathrm{PrOH}:$ hexane $=1: 500: 500,0.70 \mathrm{~mL} / \mathrm{min}$ ) to determine the retention time and enantiomeric excesses. For (+)-18, ee $=95.5 \%$.
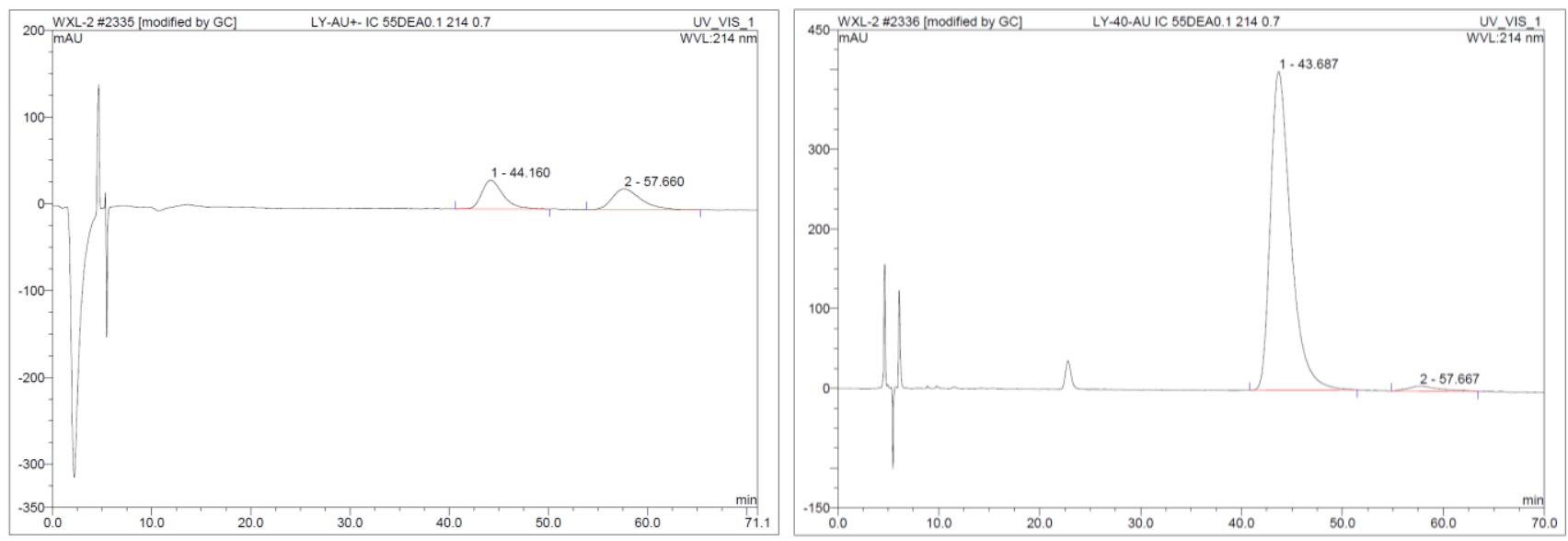

\begin{tabular}{|r|cccrrrr|}
\hline No. & $\begin{array}{c}\text { Ret.Time } \\
\text { min }\end{array}$ & Peak Name & $\begin{array}{c}\text { Height } \\
\text { mAU }\end{array}$ & $\begin{array}{c}\text { Area } \\
\text { mAU*min }\end{array}$ & $\begin{array}{r}\text { Rel.Area } \\
\%\end{array}$ & Amount & Type \\
\hline 1 & 44.16 & n.a. & 33.099 & 81.743 & 50.93 & n.a. & BMB $^{*}$ \\
2 & 57.66 & n.a. & 23.762 & 78.772 & 49.07 & n.a. & BMB $^{*}$ \\
\hline Total: & & & 56.860 & 160.514 & 100.00 & 0.000 & \\
\hline
\end{tabular}

\begin{tabular}{|r|rrrrrrr|}
\hline No. & $\begin{array}{c}\text { Ret.Time } \\
\text { min }\end{array}$ & Peak Name & $\begin{array}{c}\text { Height } \\
\text { mAU }\end{array}$ & $\begin{array}{c}\text { Area } \\
\text { mAU*min }\end{array}$ & $\begin{array}{r}\text { Rel.Area } \\
\%\end{array}$ & Amount & Type \\
\hline 1 & 43.69 & n.a. & 399.416 & 934.899 & 97.77 & n.a. & BMB* $^{*}$ \\
2 & 57.67 & n.a. & 6.168 & 21.327 & 2.23 & n.a. & BMB* $^{*}$ \\
\hline Total: & & & 405.584 & 956.226 & 100.00 & 0.000 & \\
\hline
\end{tabular}


IV ${ }^{1} \mathrm{H}$ and ${ }^{13} \mathrm{C}$ NMR Spectra of Compounds

${ }^{1} \mathrm{H}$ NMR Spectrum of $16\left(500 \mathrm{MHz}, \mathrm{CDCl}_{3}\right)$

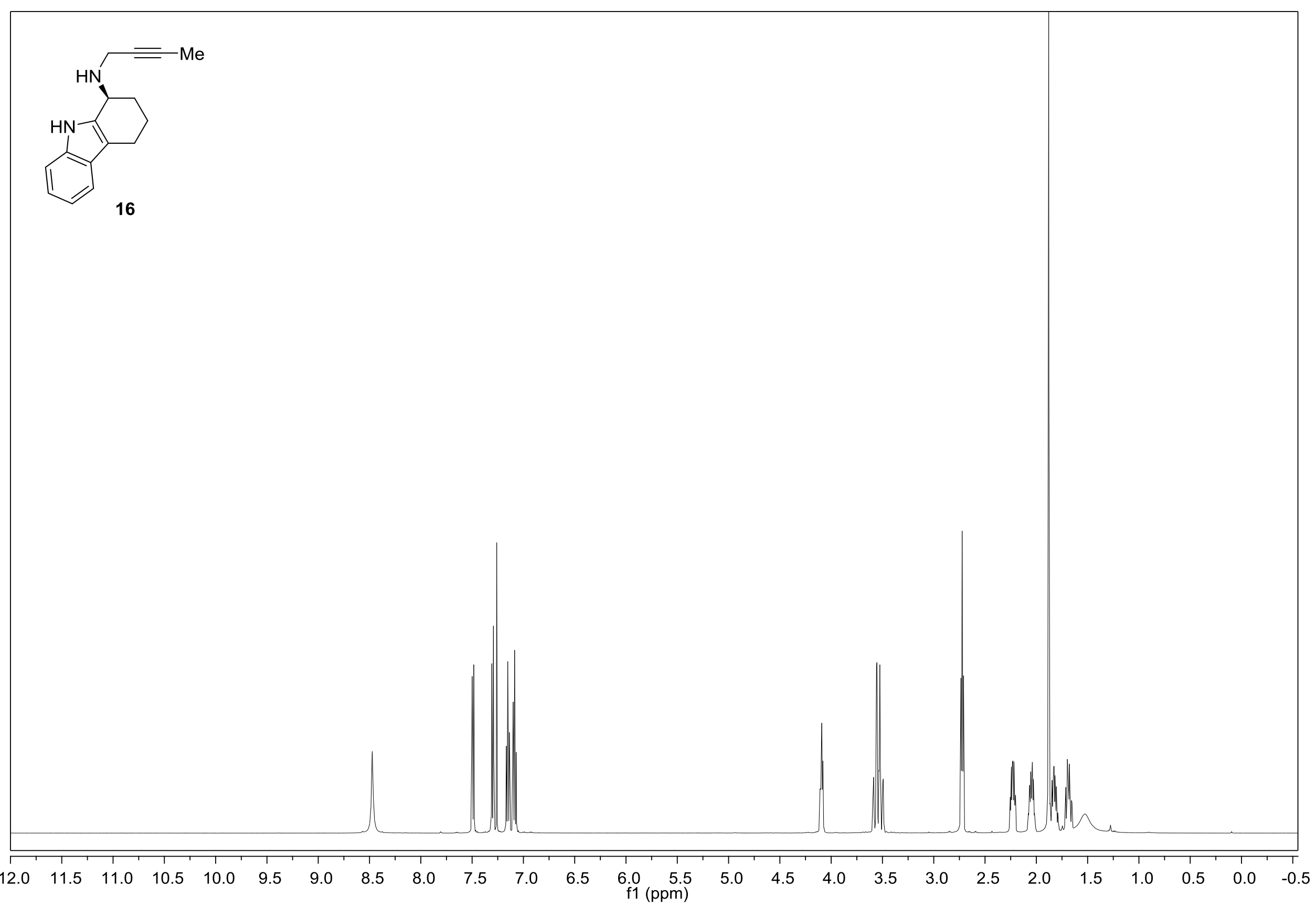




\section{${ }^{13} \mathrm{C}$ NMR Spectrum of $16\left(126 \mathrm{MHz}, \mathrm{CDCl}_{3}\right)$}

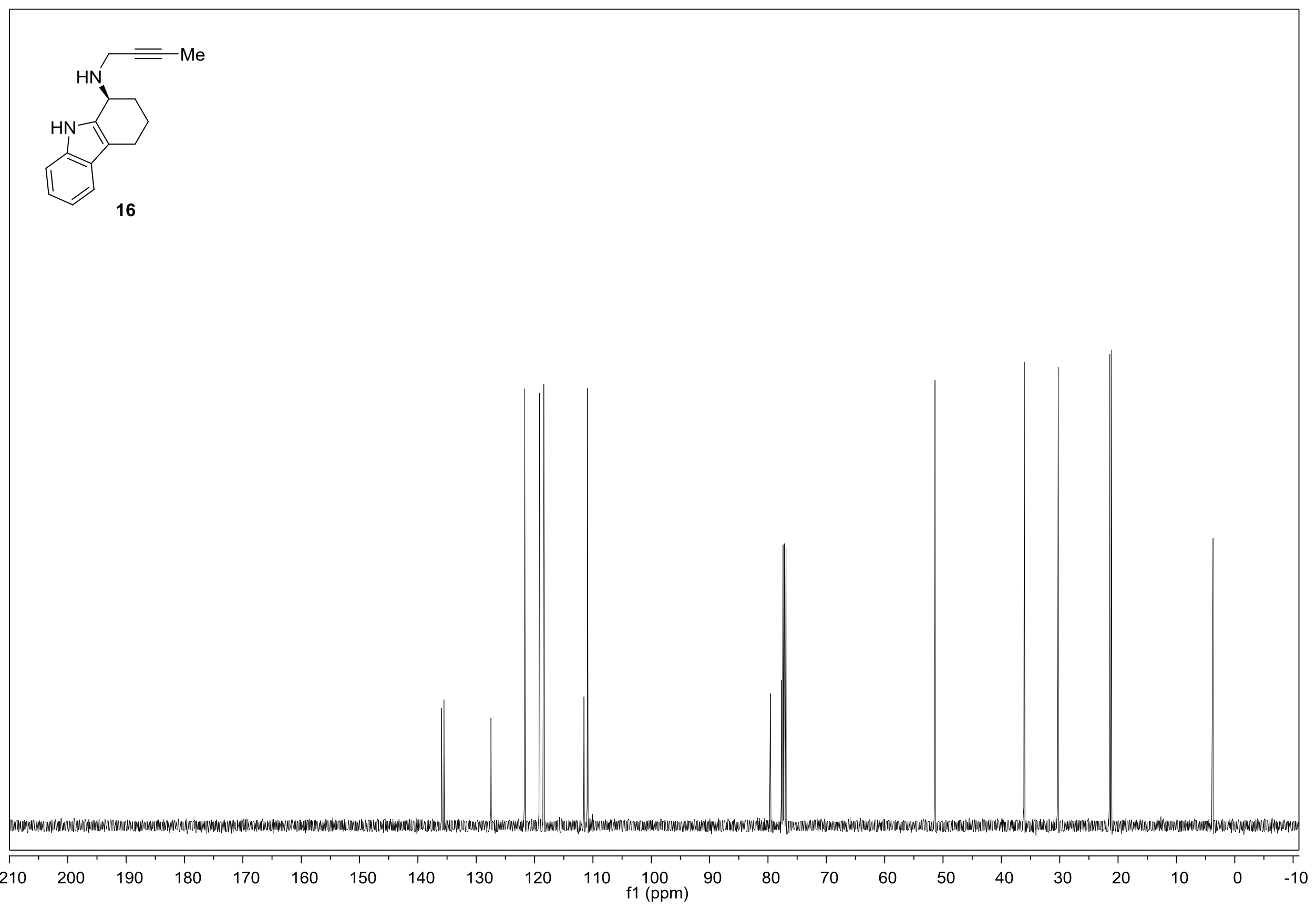


${ }^{1} \mathrm{H}$ NMR Spectrum of $17\left(500 \mathrm{MHz}, \mathrm{CDCl}_{3}\right)$

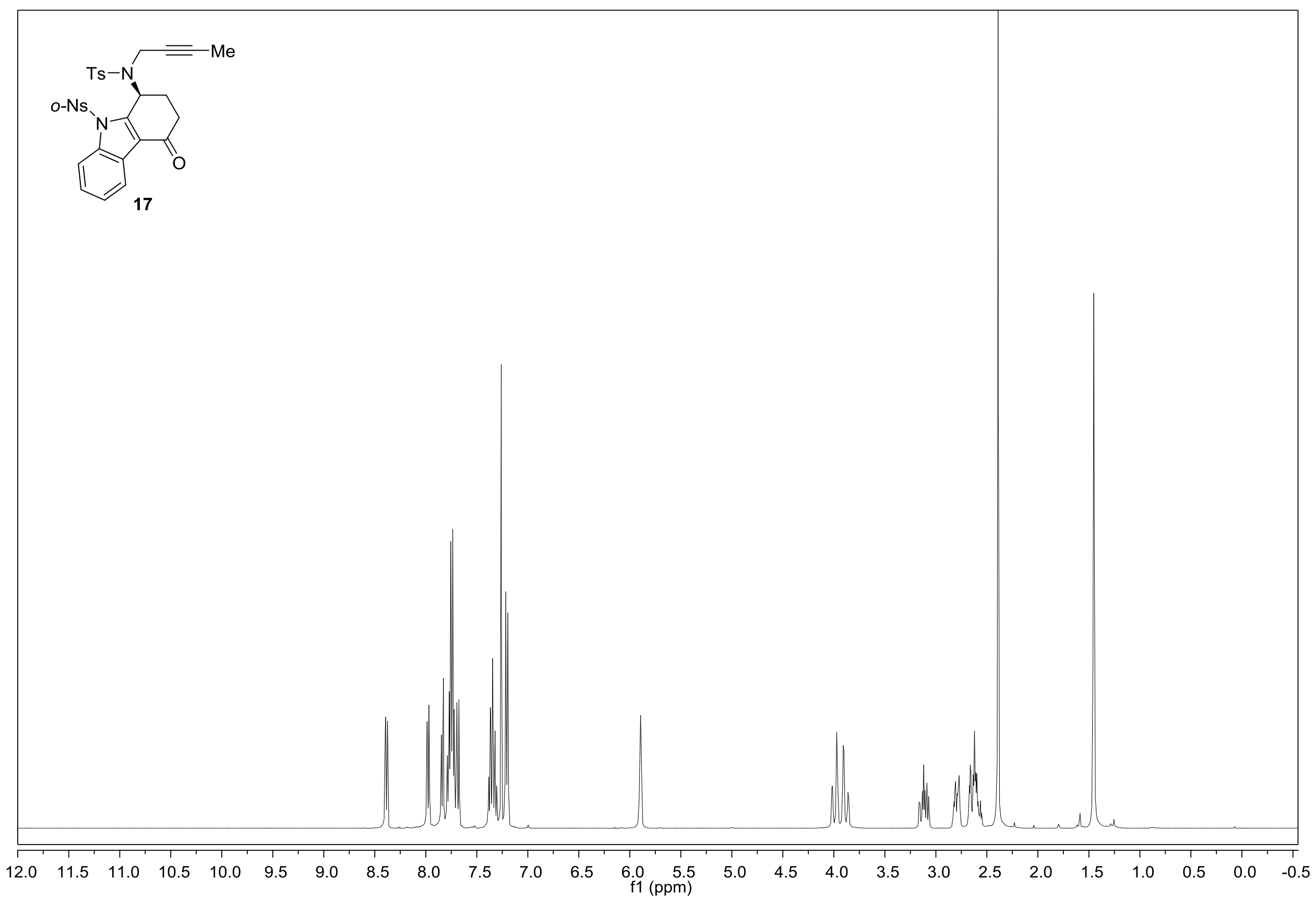


${ }^{13} \mathrm{C}$ NMR Spectrum of $17\left(126 \mathrm{MHz}, \mathrm{CDCl}_{3}\right)$

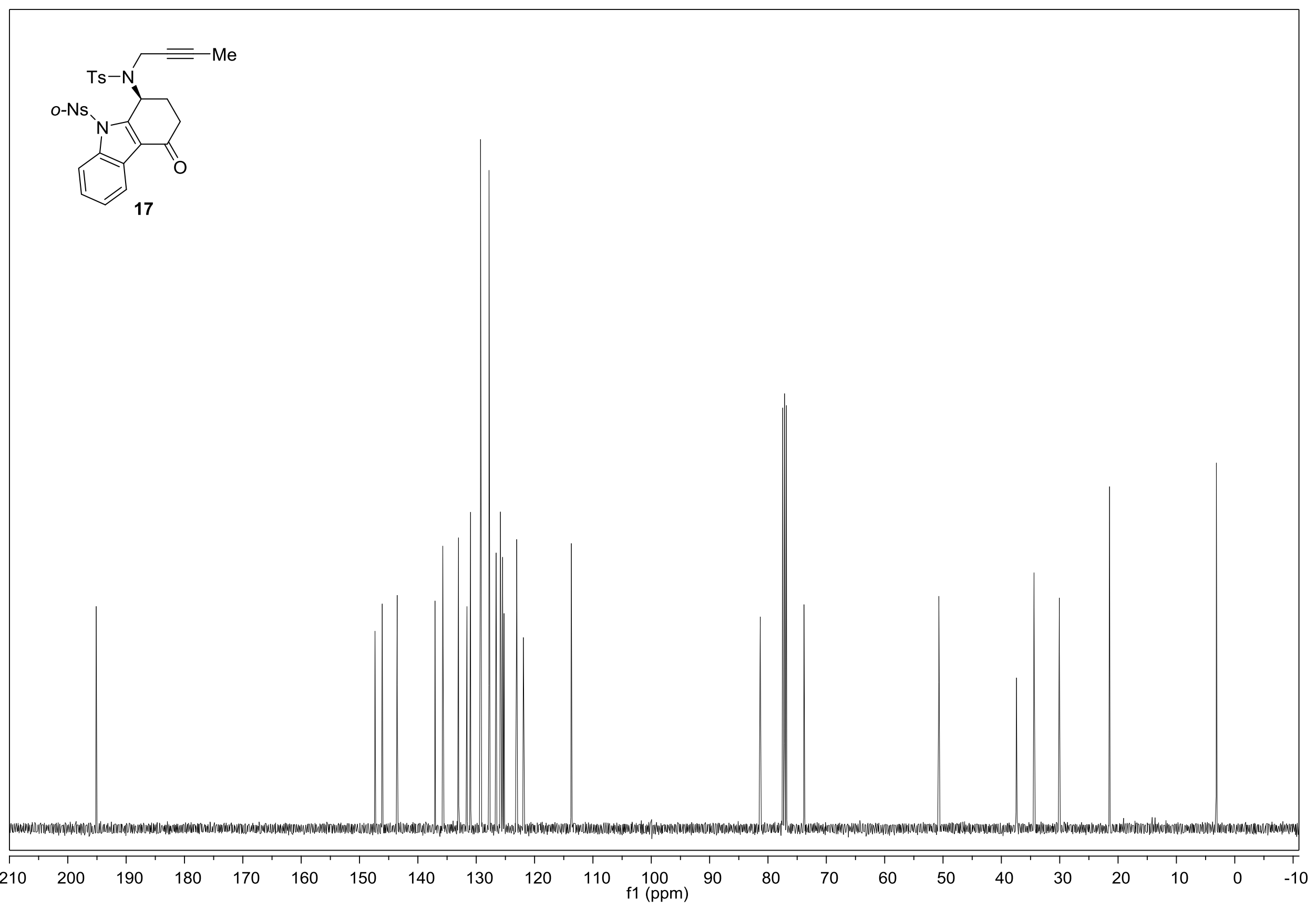


${ }^{1} \mathrm{H}$ NMR Spectrum of $18\left(500 \mathrm{MHz}, \mathrm{CDCl}_{3}\right)$

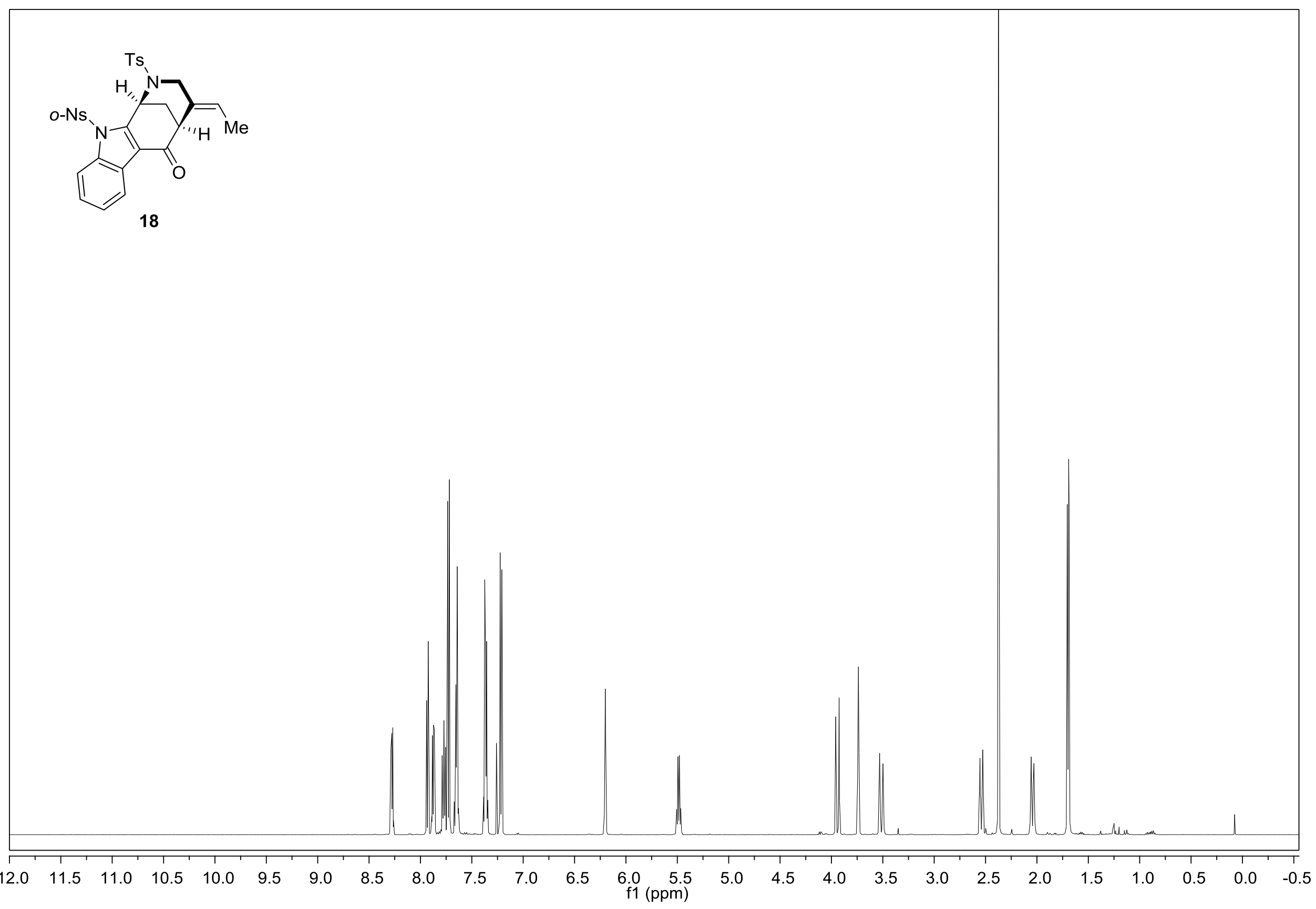




\section{${ }^{13} \mathrm{C}$ NMR Spectrum of $18\left(126 \mathrm{MHz}, \mathrm{CDCl}_{3}\right)$}

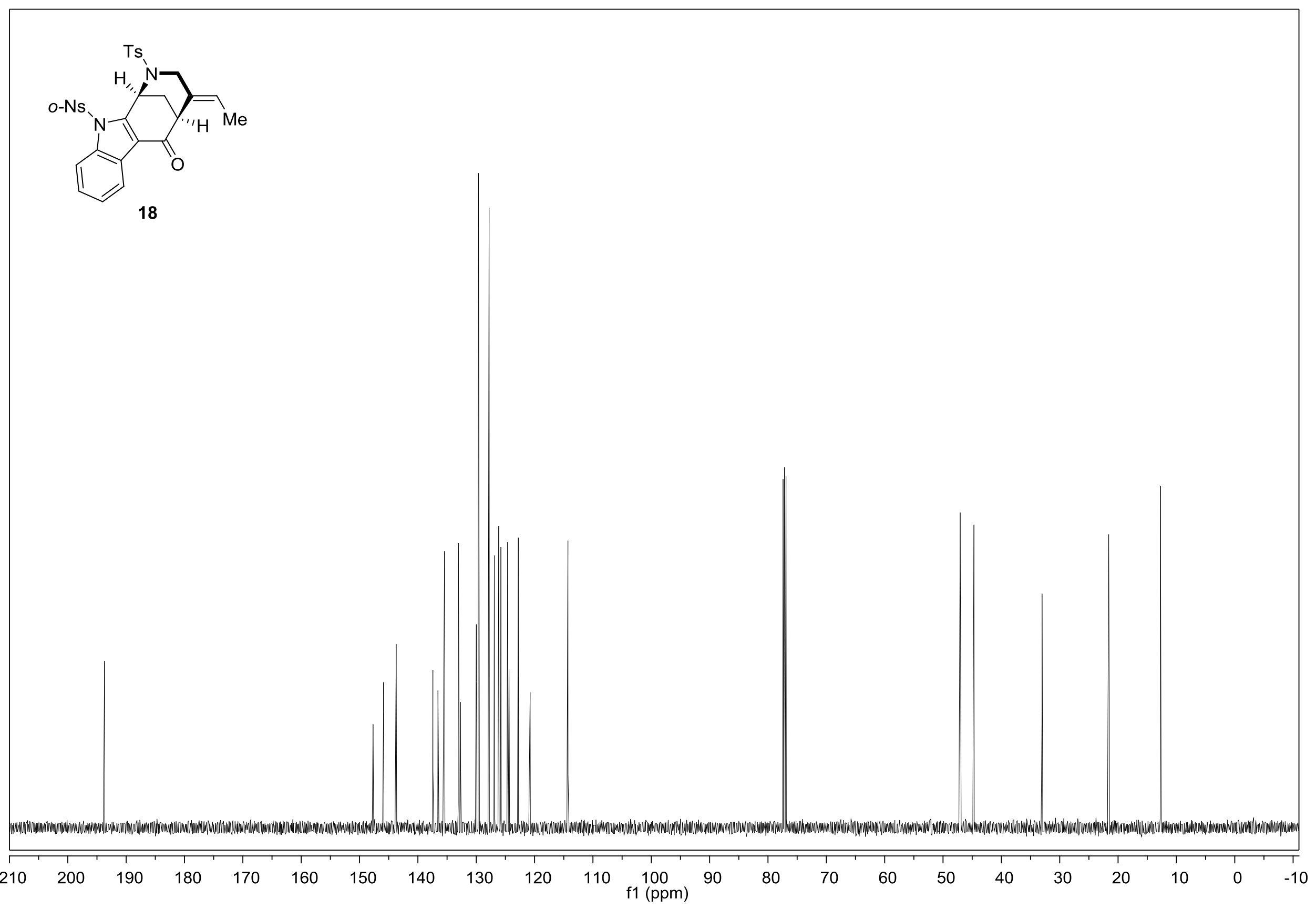


${ }^{1} \mathrm{H}$ NMR Spectrum of $20\left(500 \mathrm{MHz}, \mathrm{CDCl}_{3}\right)$

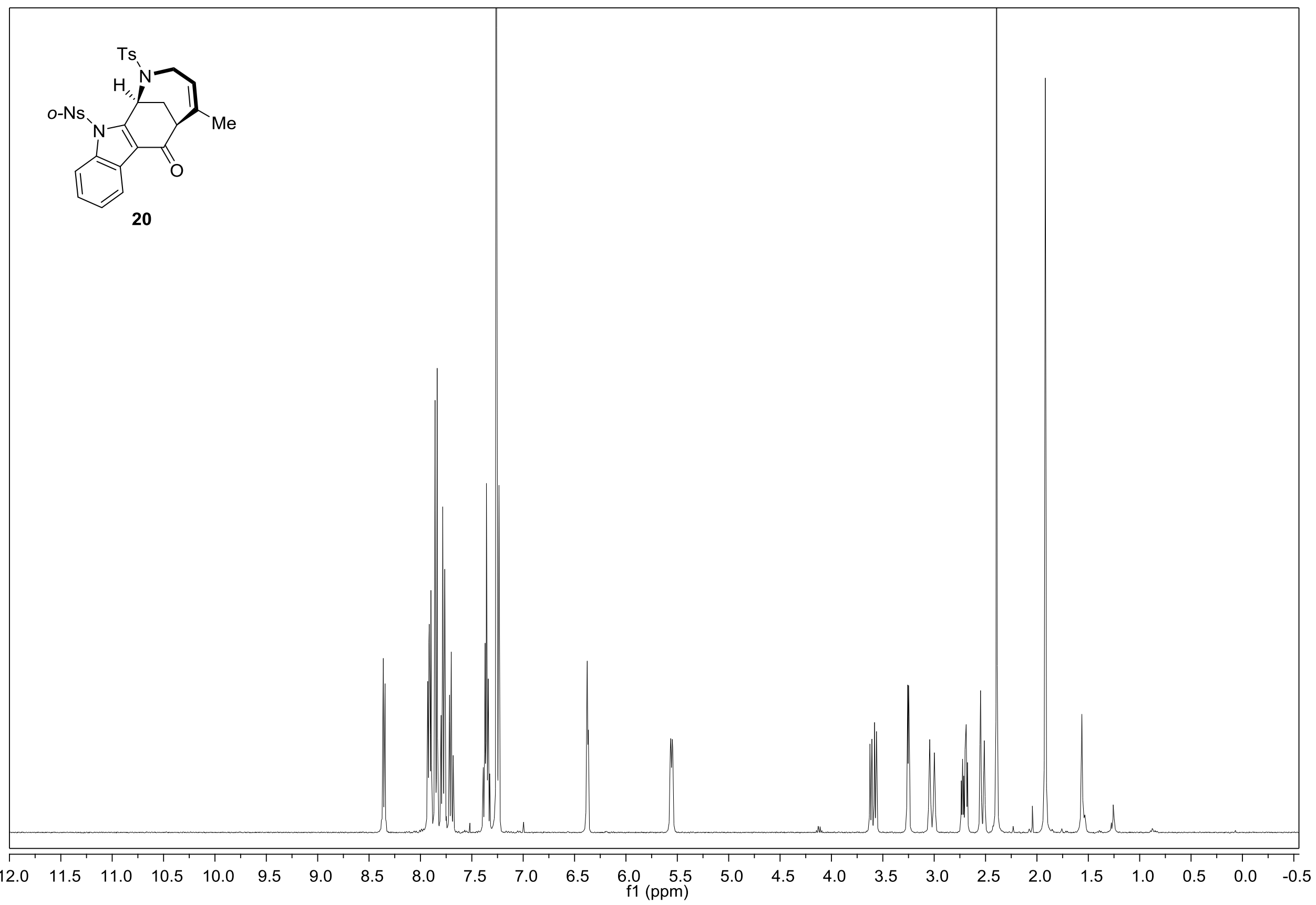


${ }^{13} \mathrm{C}$ NMR Spectrum of $20\left(126 \mathrm{MHz}, \mathrm{CDCl}_{3}\right)$

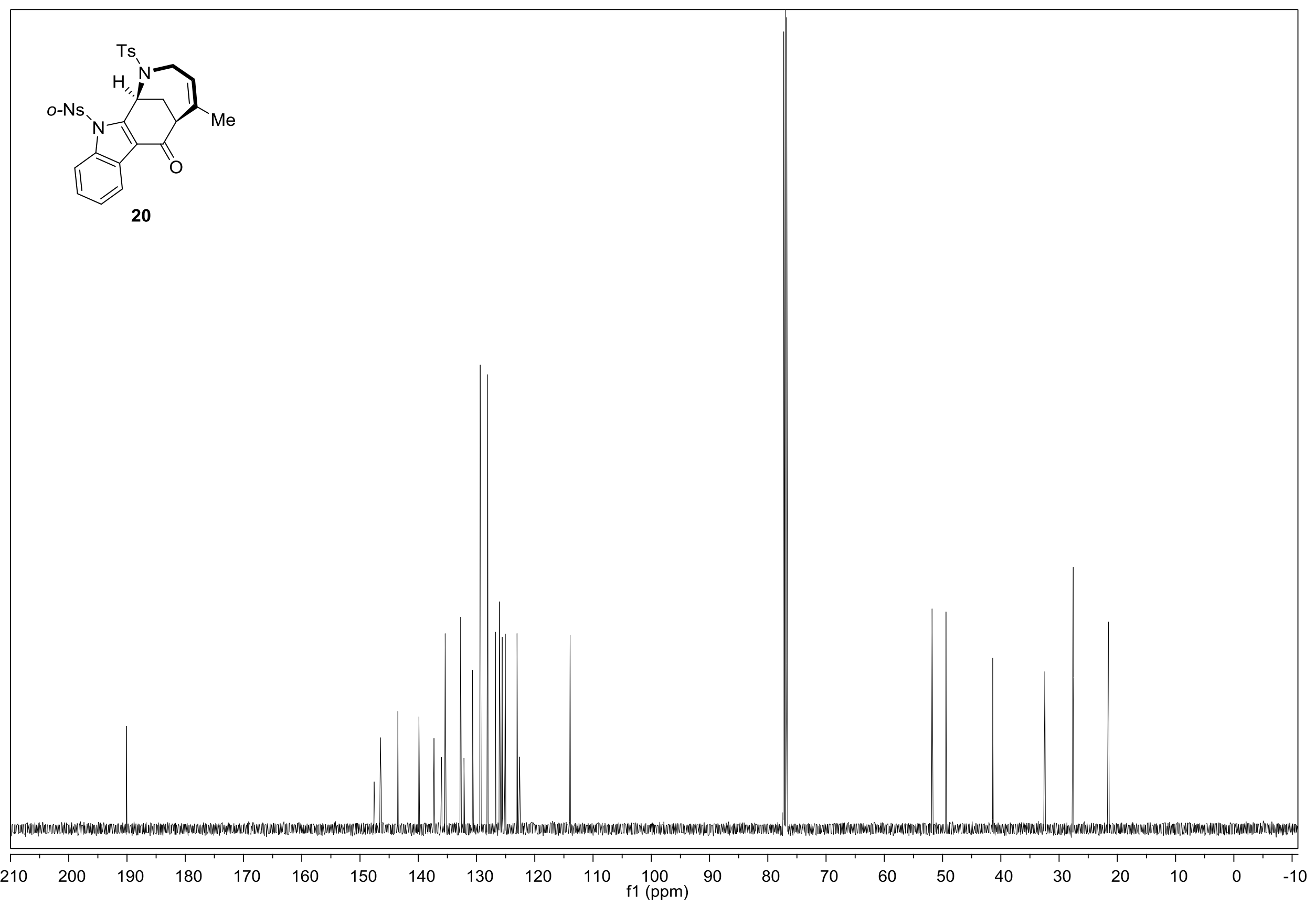


${ }^{1} \mathrm{H}$ NMR Spectrum of $22\left(500 \mathrm{MHz}, \mathrm{CDCl}_{3}\right)$

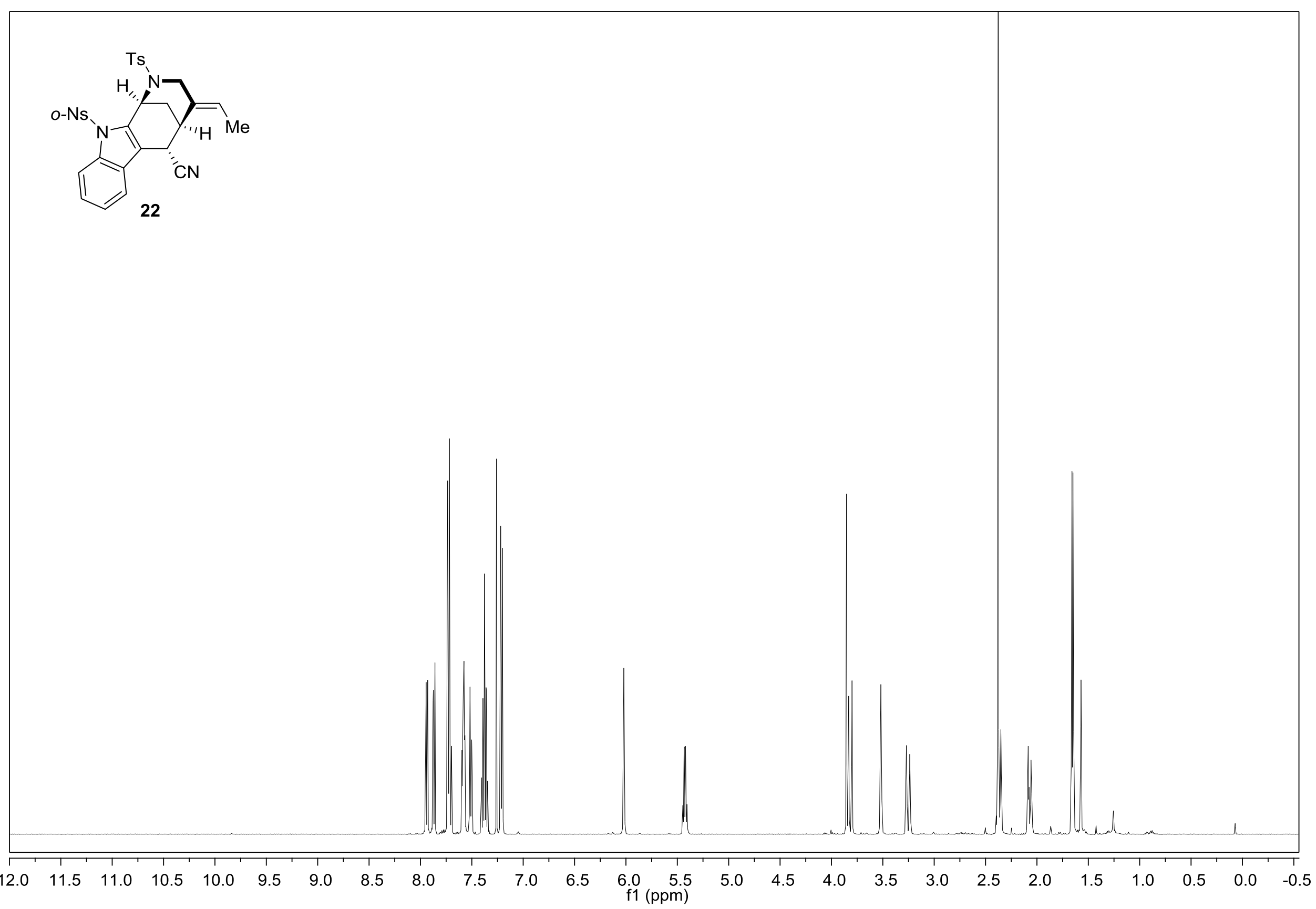


${ }^{13} \mathrm{C}$ NMR Spectrum of $22\left(101 \mathrm{MHz}, \mathrm{CDCl}_{3}\right)$

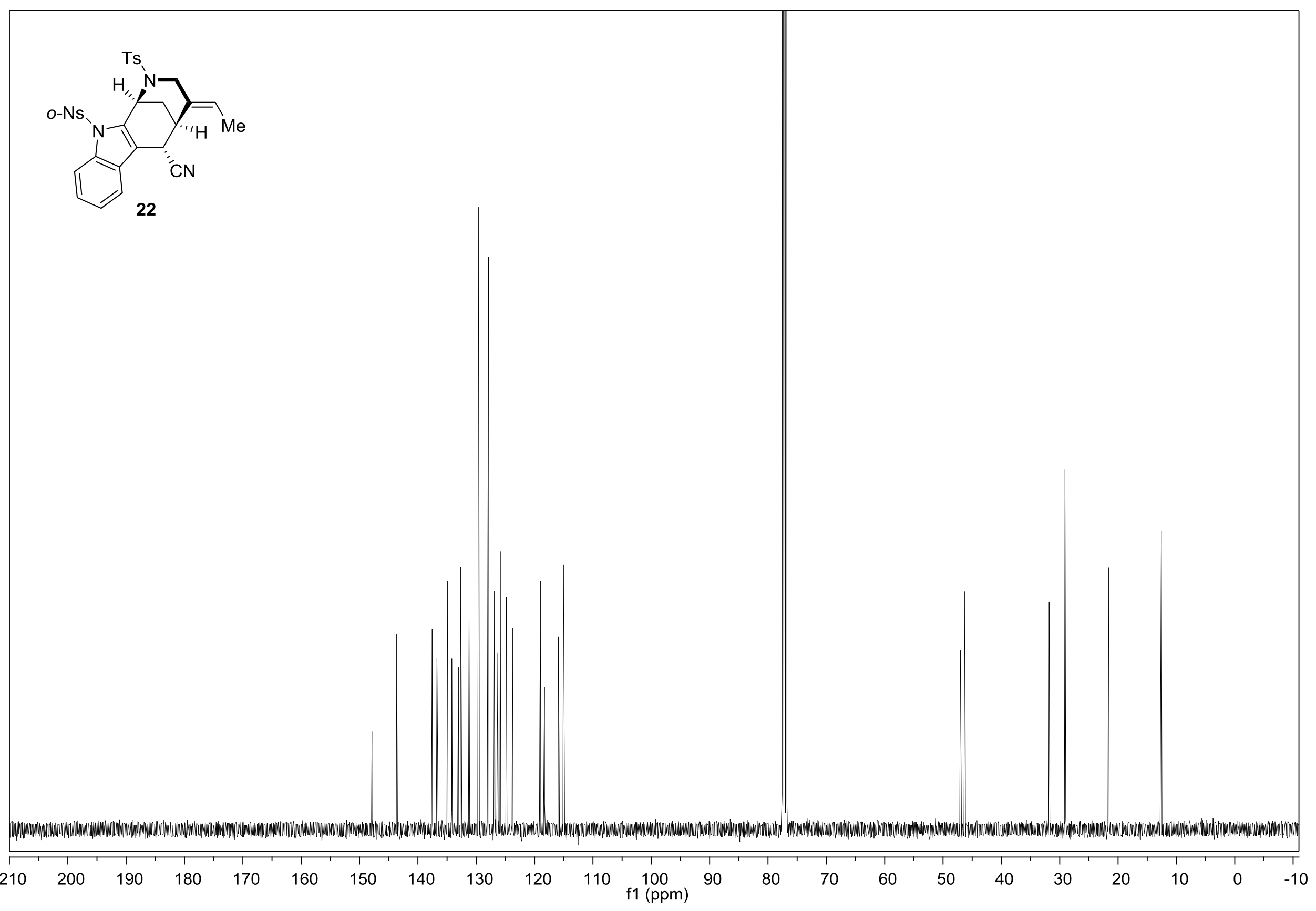


${ }^{1} \mathrm{H}$ NMR Spectrum of $23\left(400 \mathrm{MHz}, \mathrm{CDCl}_{3}\right)$

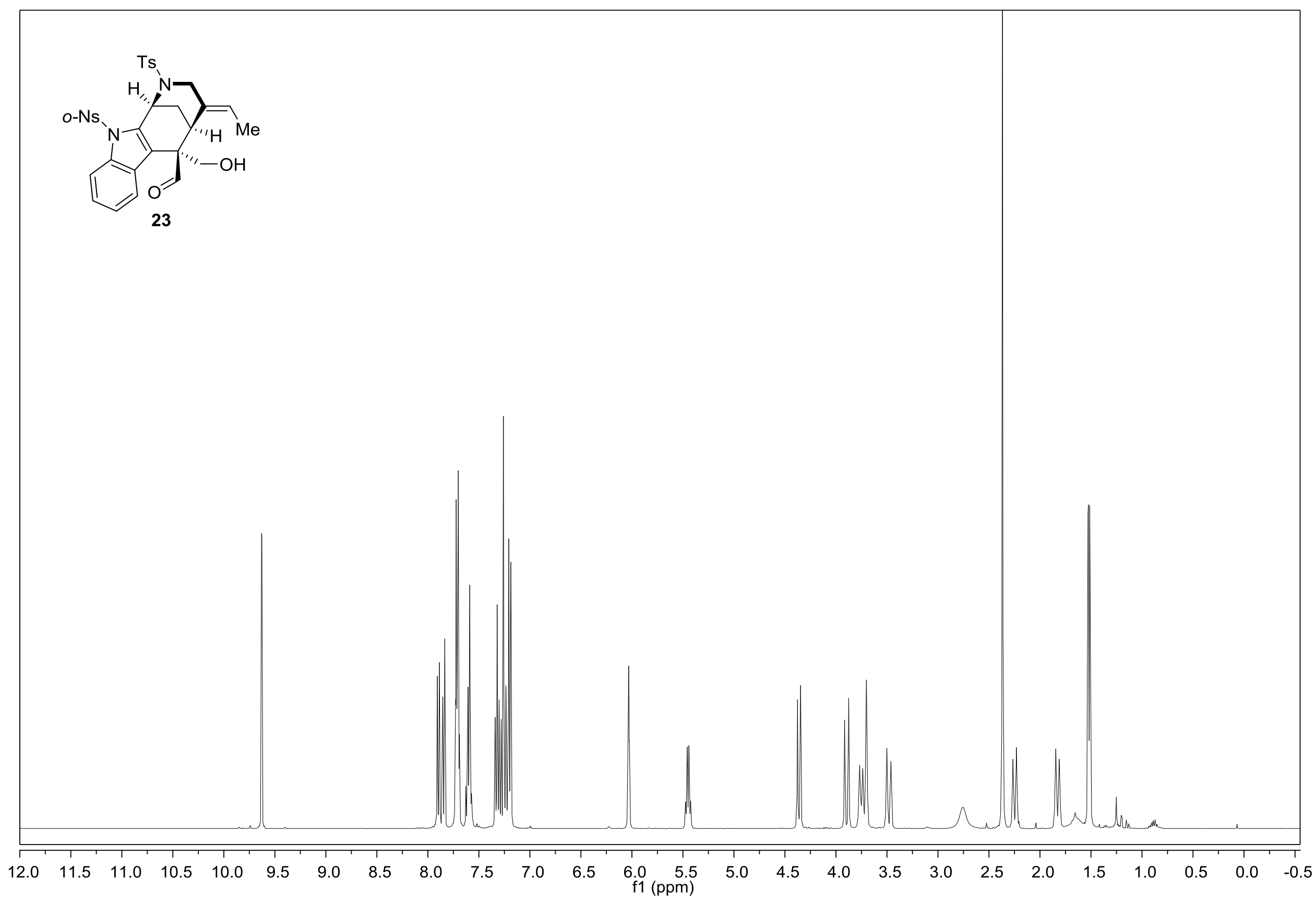


${ }^{13} \mathrm{C}$ NMR Spectrum of $23\left(126 \mathrm{MHz}, \mathrm{CDCl}_{3}\right)$

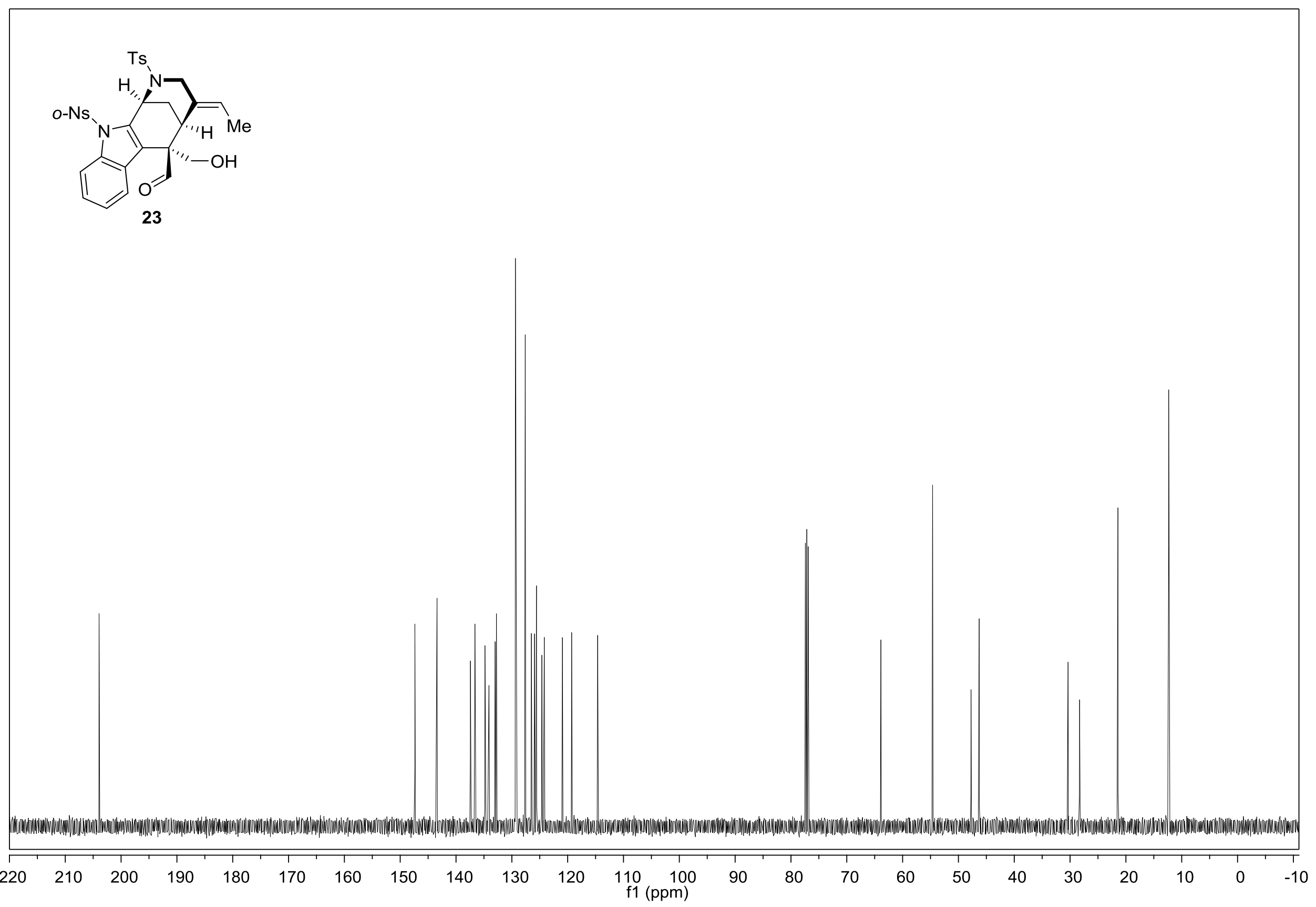


${ }^{1} \mathrm{H}$ NMR Spectrum of $24\left(400 \mathrm{MHz}, \mathrm{CDCl}_{3}\right)$

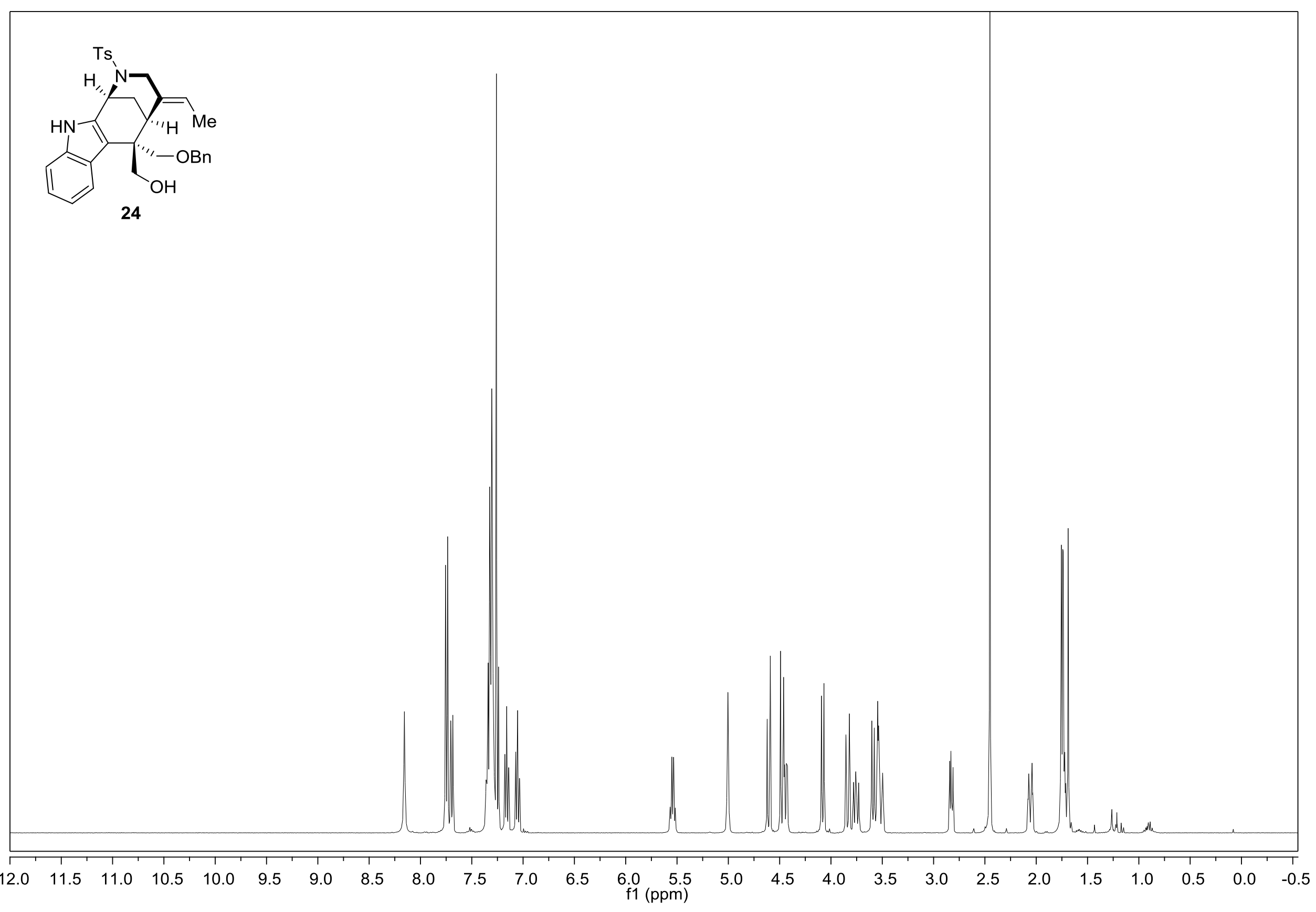


${ }^{13} \mathrm{C}$ NMR Spectrum of $24\left(126 \mathrm{MHz}, \mathrm{CDCl}_{3}\right)$

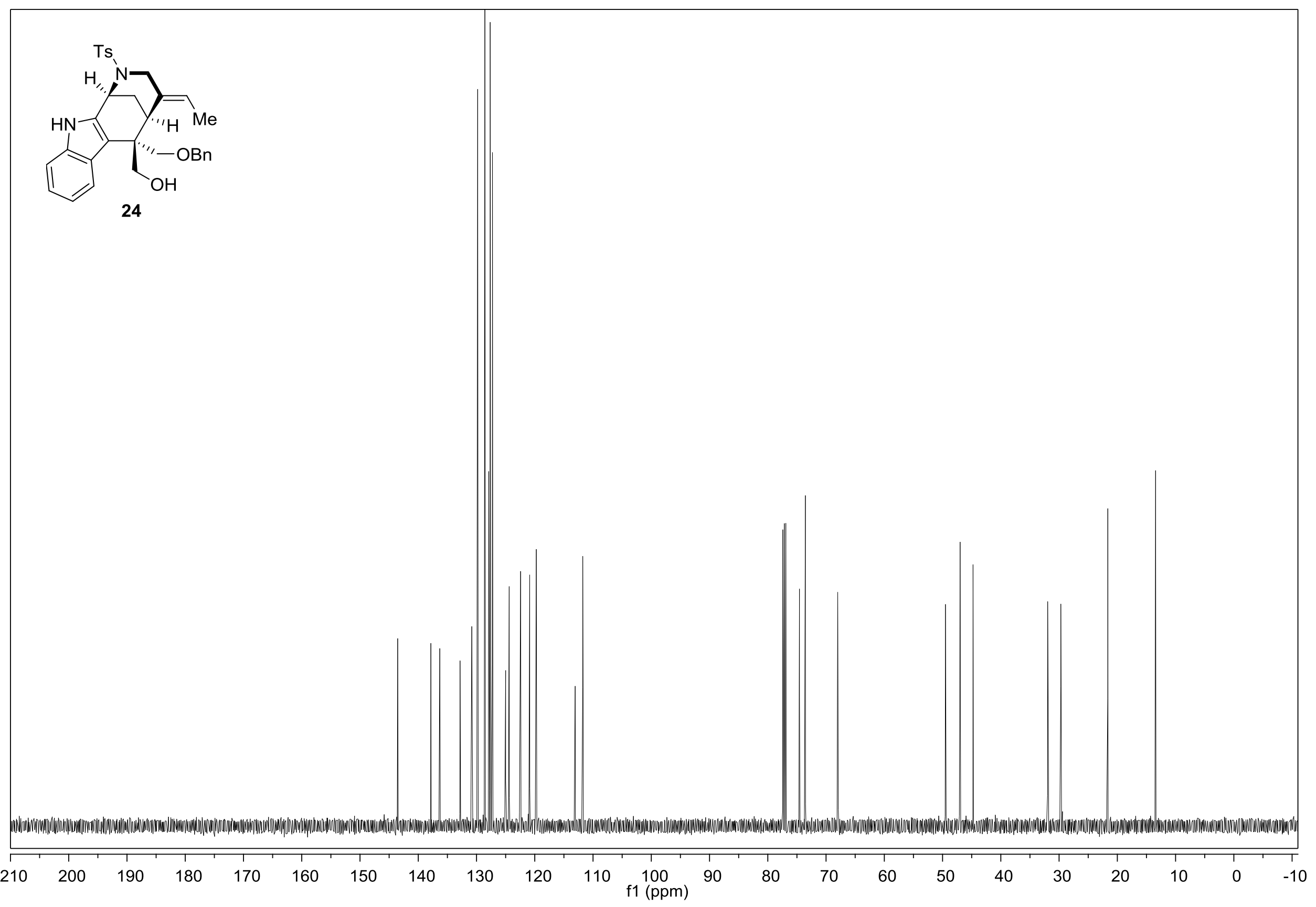




\section{${ }^{1} \mathrm{H}$ NMR Spectrum of $26\left(500 \mathrm{MHz}, \mathrm{CDCl}_{3}\right)$}

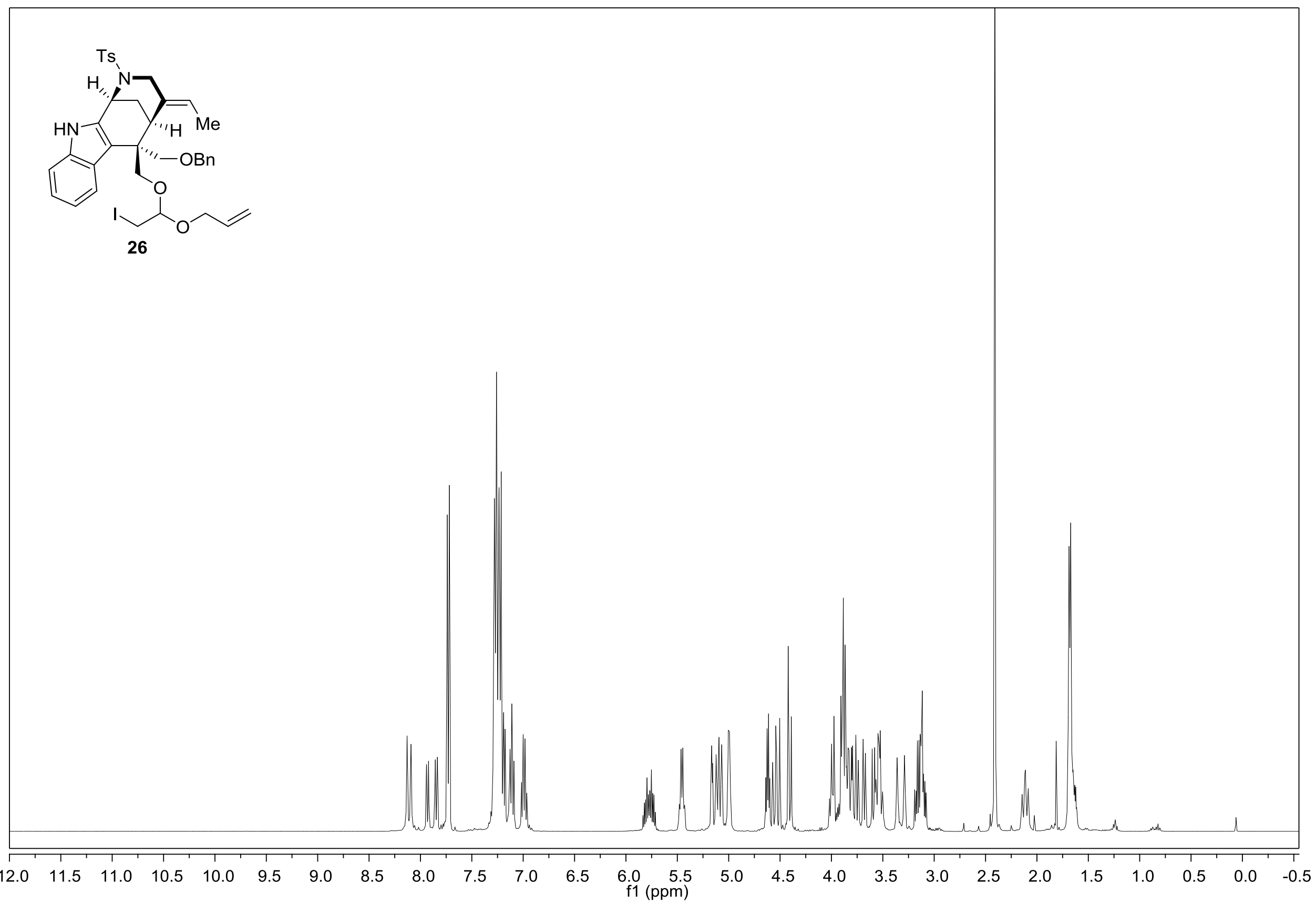




\section{${ }^{13} \mathrm{C}$ NMR Spectrum of $26\left(126 \mathrm{MHz}, \mathrm{CDCl}_{3}\right)$}

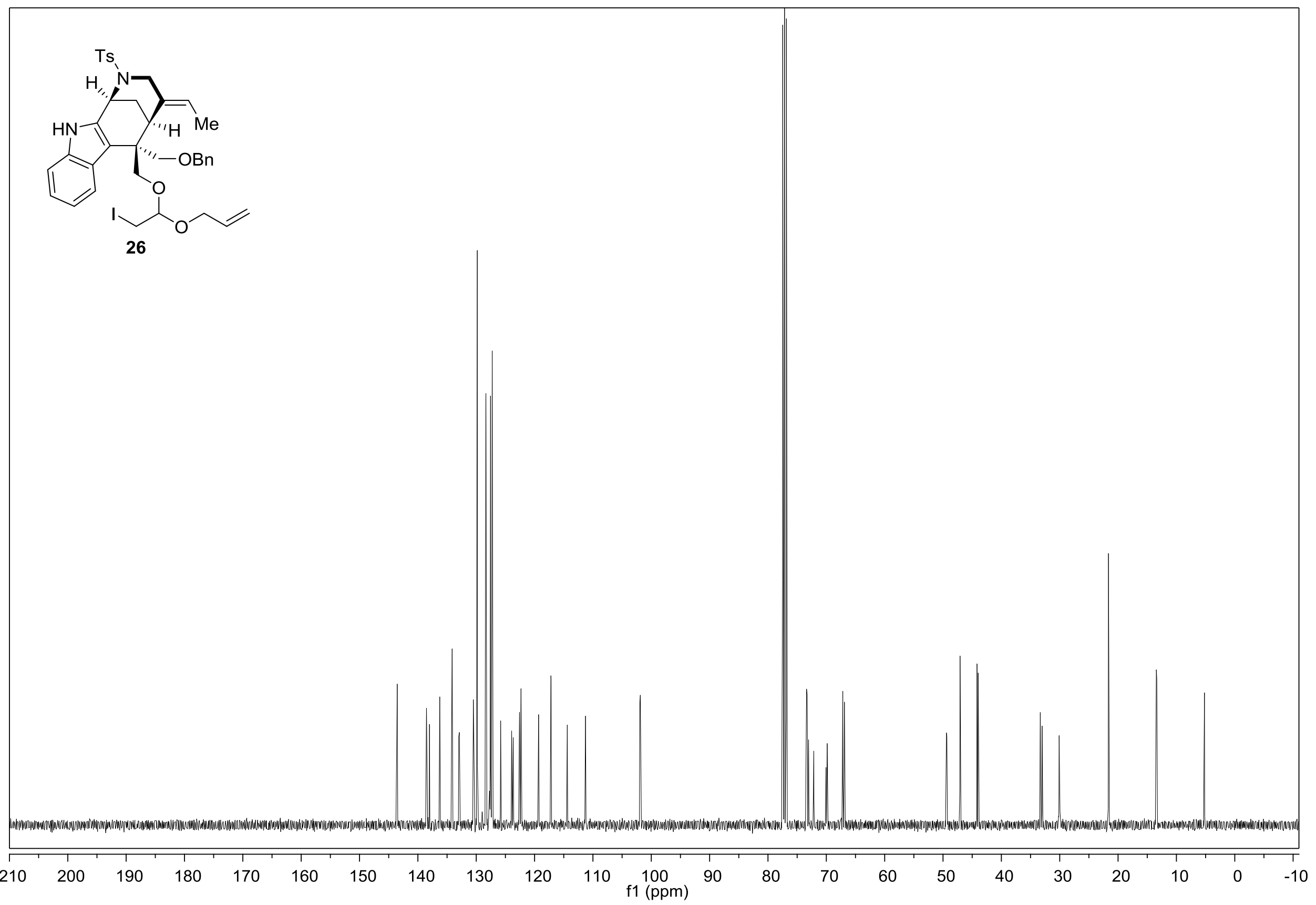


${ }^{1} \mathrm{H}$ NMR Spectrum of $27 \mathrm{a}\left(400 \mathrm{MHz}, \mathrm{CDCl}_{3}\right)$

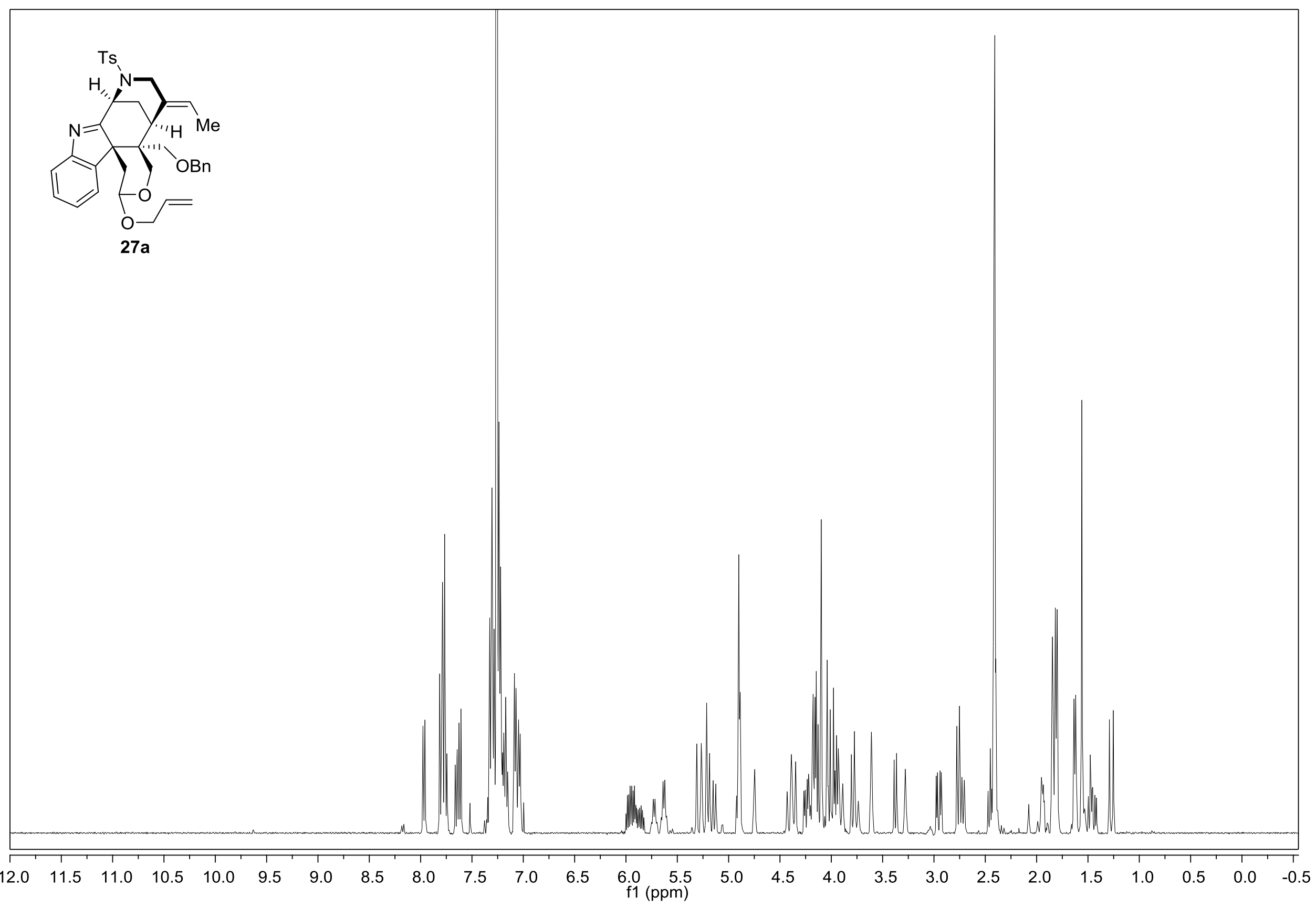


${ }^{13} \mathrm{C}$ NMR Spectrum of 27a (101 $\left.\mathrm{MHz}, \mathrm{CDCl}_{3}\right)$

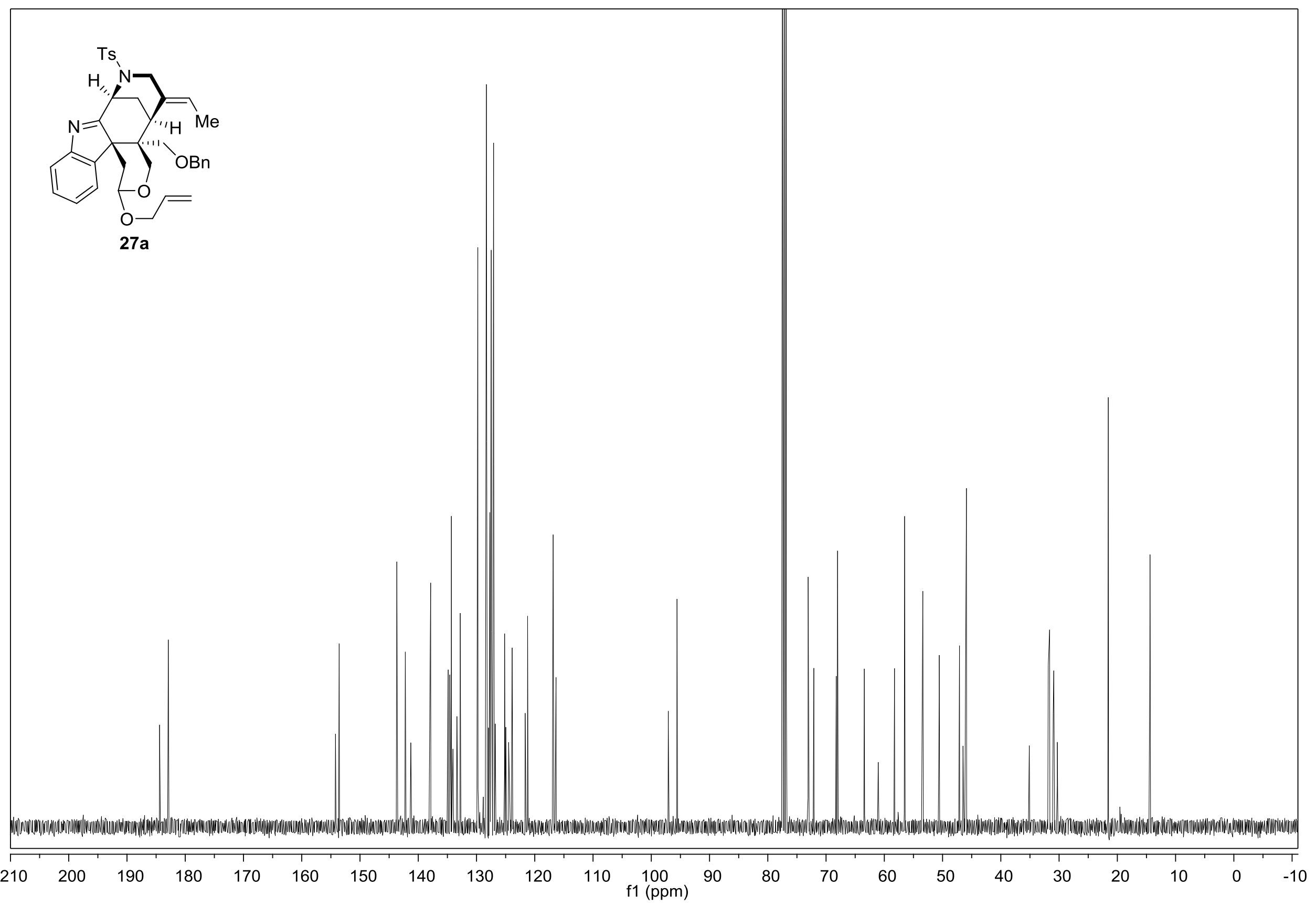


${ }^{1} \mathrm{H}$ NMR Spectrum of $27\left(500 \mathrm{MHz}, \mathrm{CDCl}_{3}\right)$

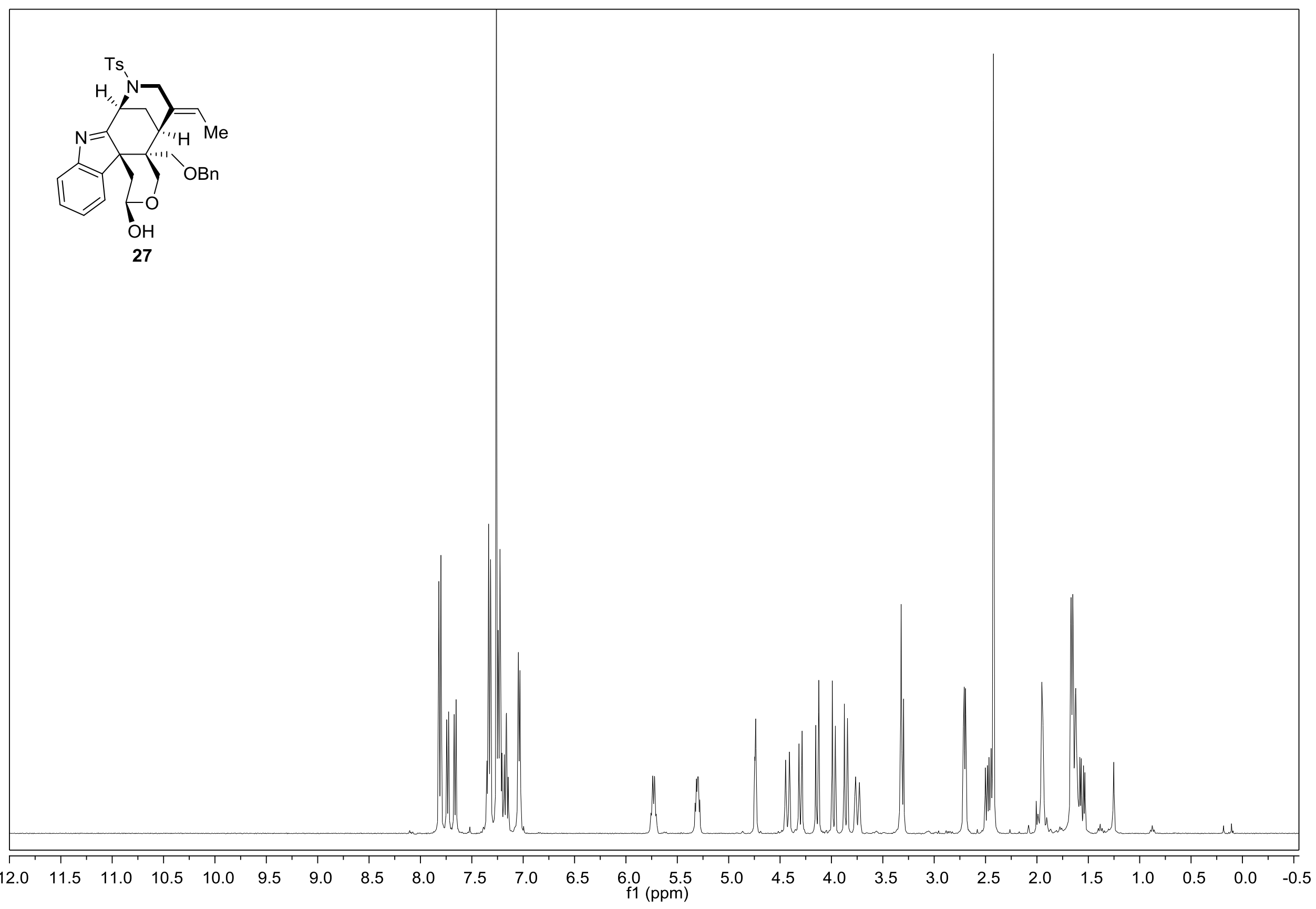


${ }^{13} \mathrm{C}$ NMR Spectrum of $27\left(101 \mathrm{MHz}, \mathrm{CDCl}_{3}\right)$

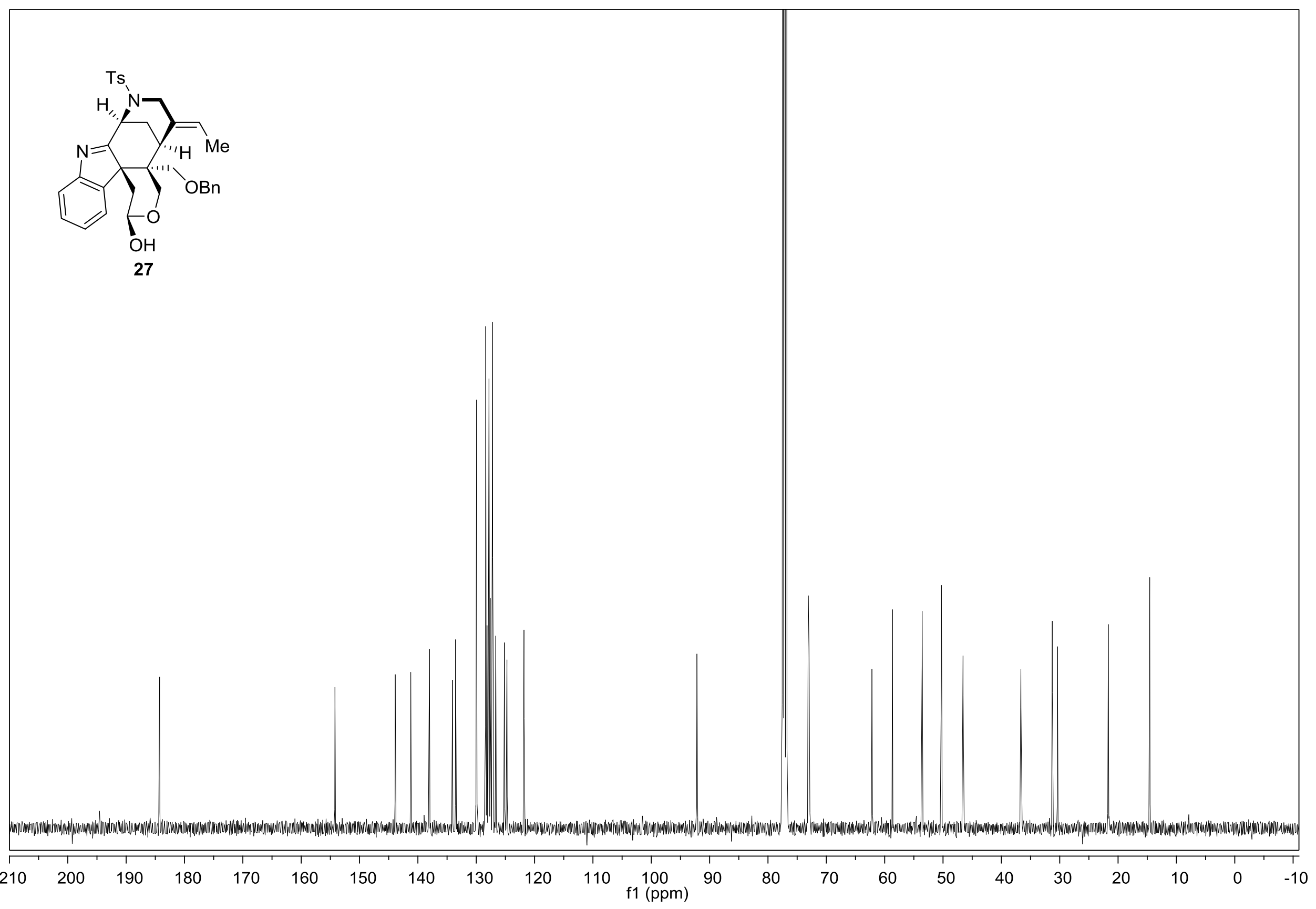




\section{${ }^{1} \mathrm{H}$ NMR Spectrum of $28\left(400 \mathrm{MHz}, \mathrm{CDCl}_{3}\right)$}

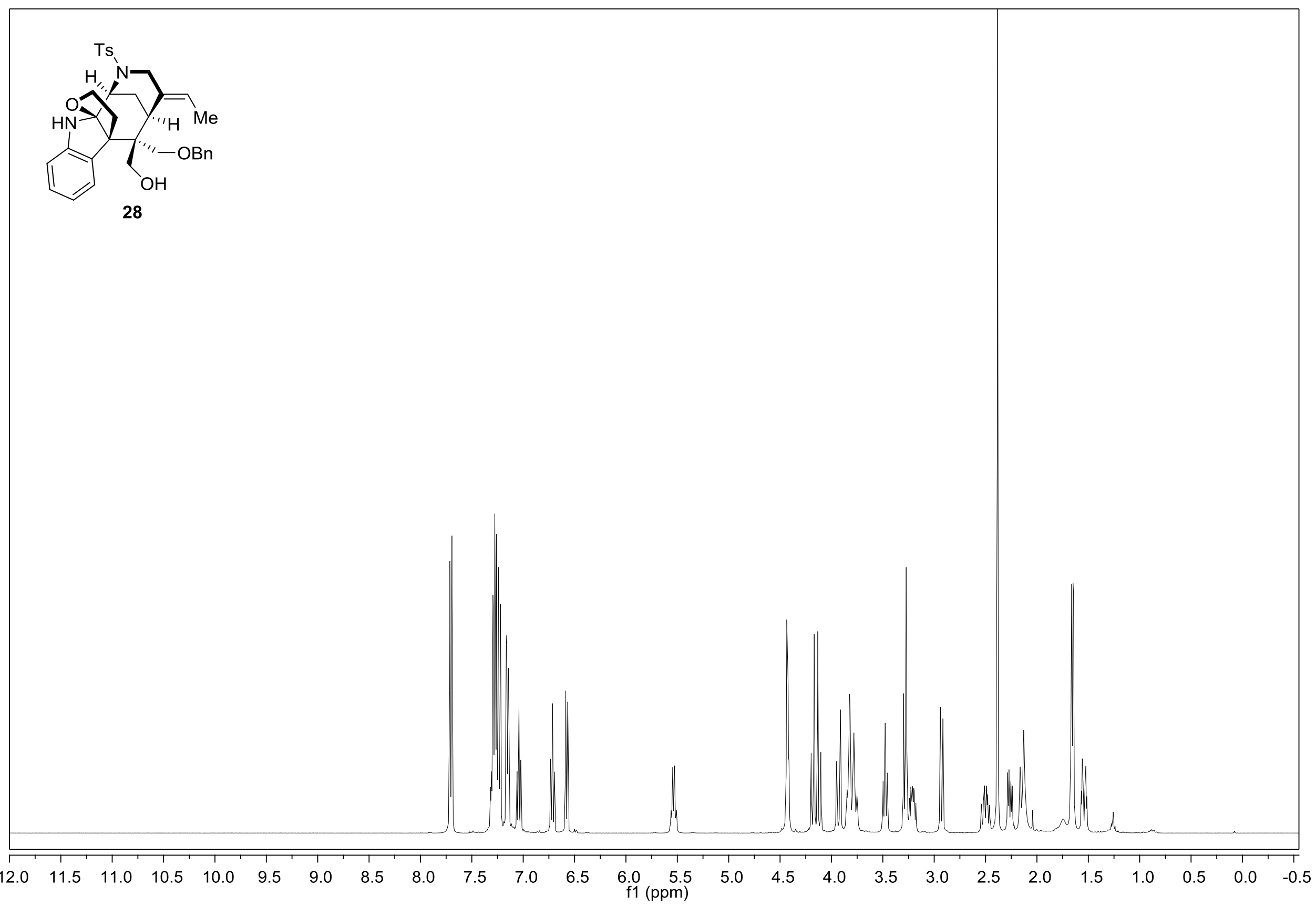


${ }^{13} \mathrm{C}$ NMR Spectrum of $28\left(126 \mathrm{MHz}, \mathrm{CDCl}_{3}\right)$

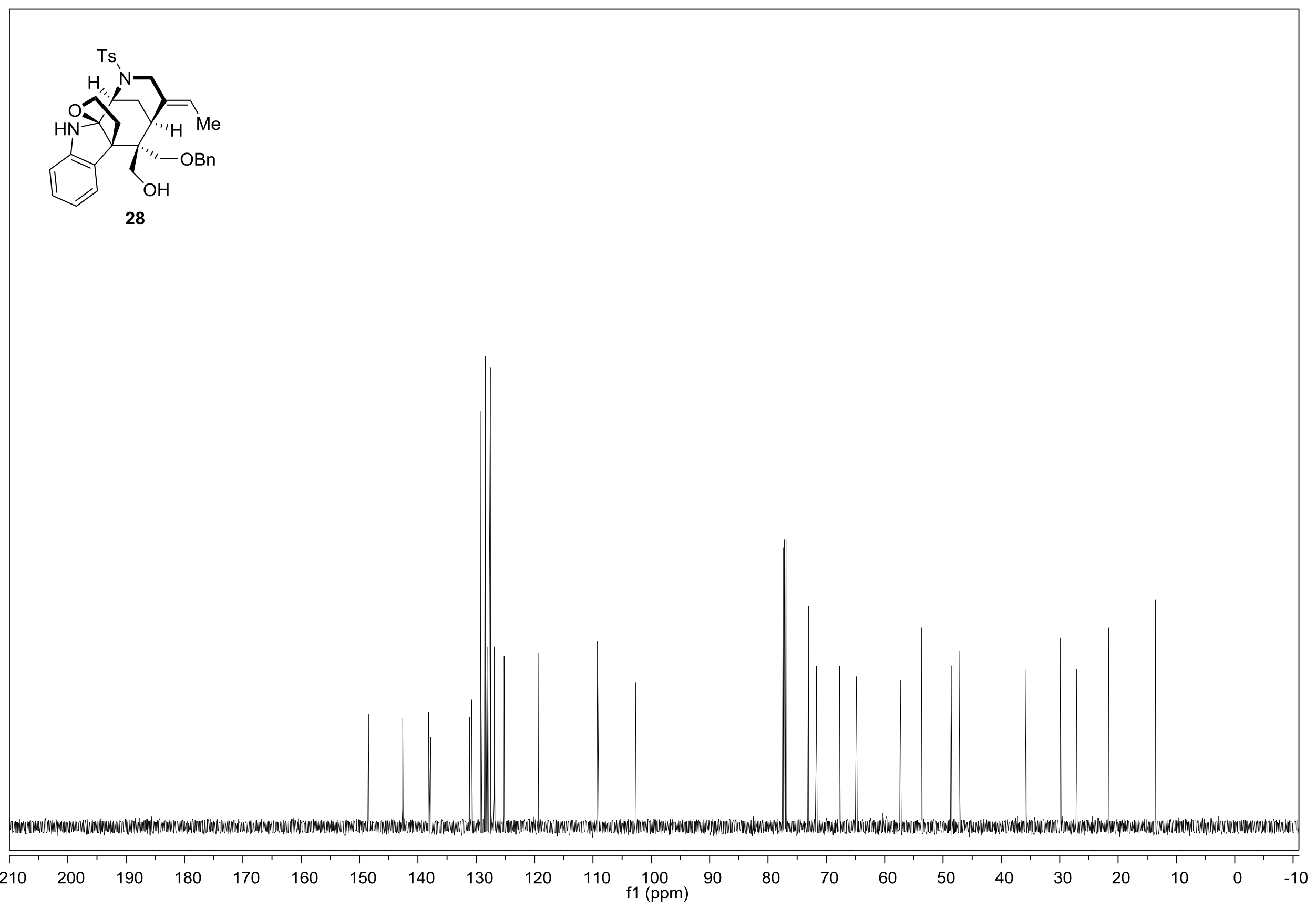


${ }^{1} \mathrm{H}$ NMR Spectrum of $29\left(400 \mathrm{MHz}, \mathrm{CDCl}_{3}\right)$

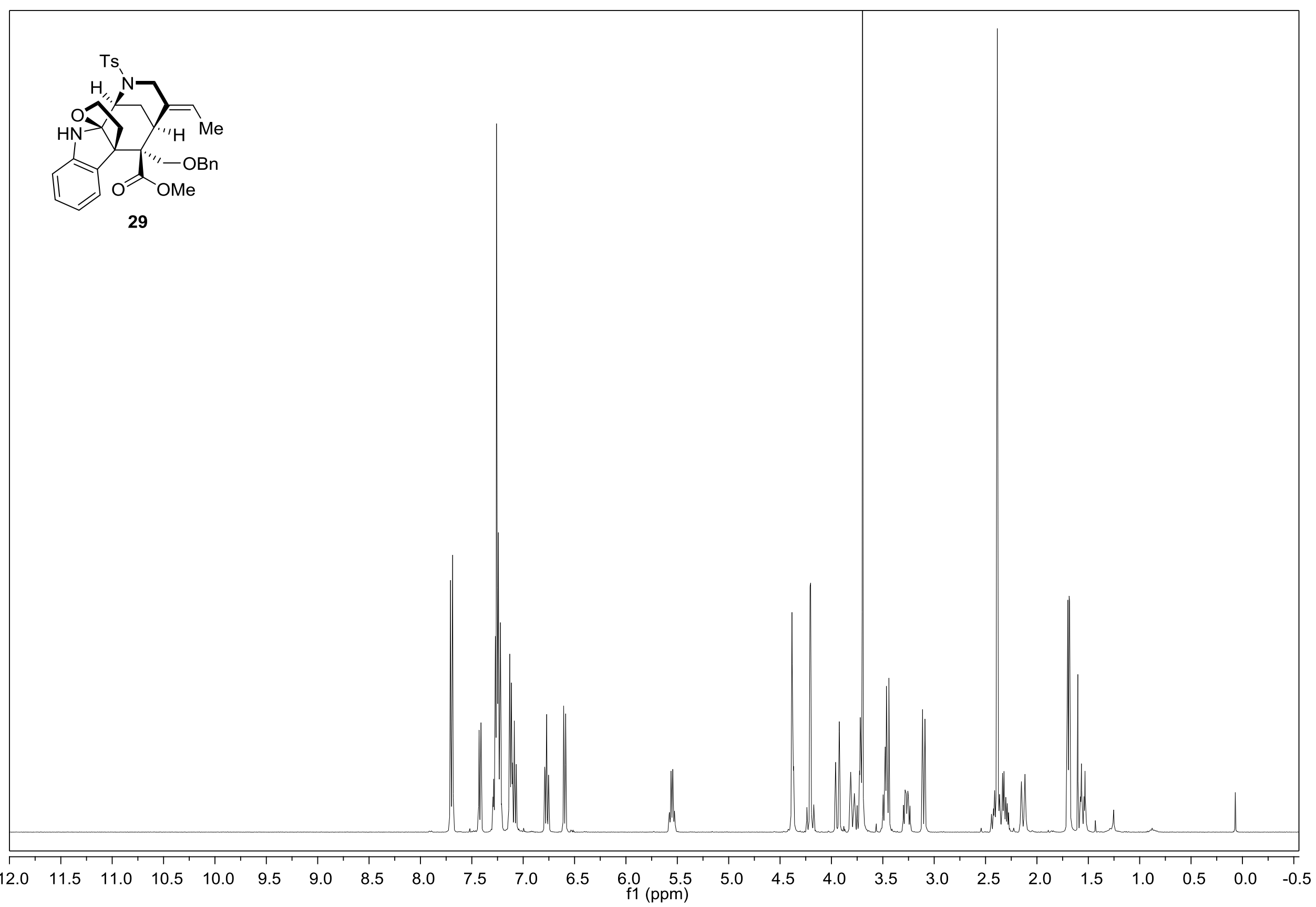




\section{${ }^{13} \mathrm{C}$ NMR Spectrum of $29\left(126 \mathrm{MHz}, \mathrm{CDCl}_{3}\right)$}

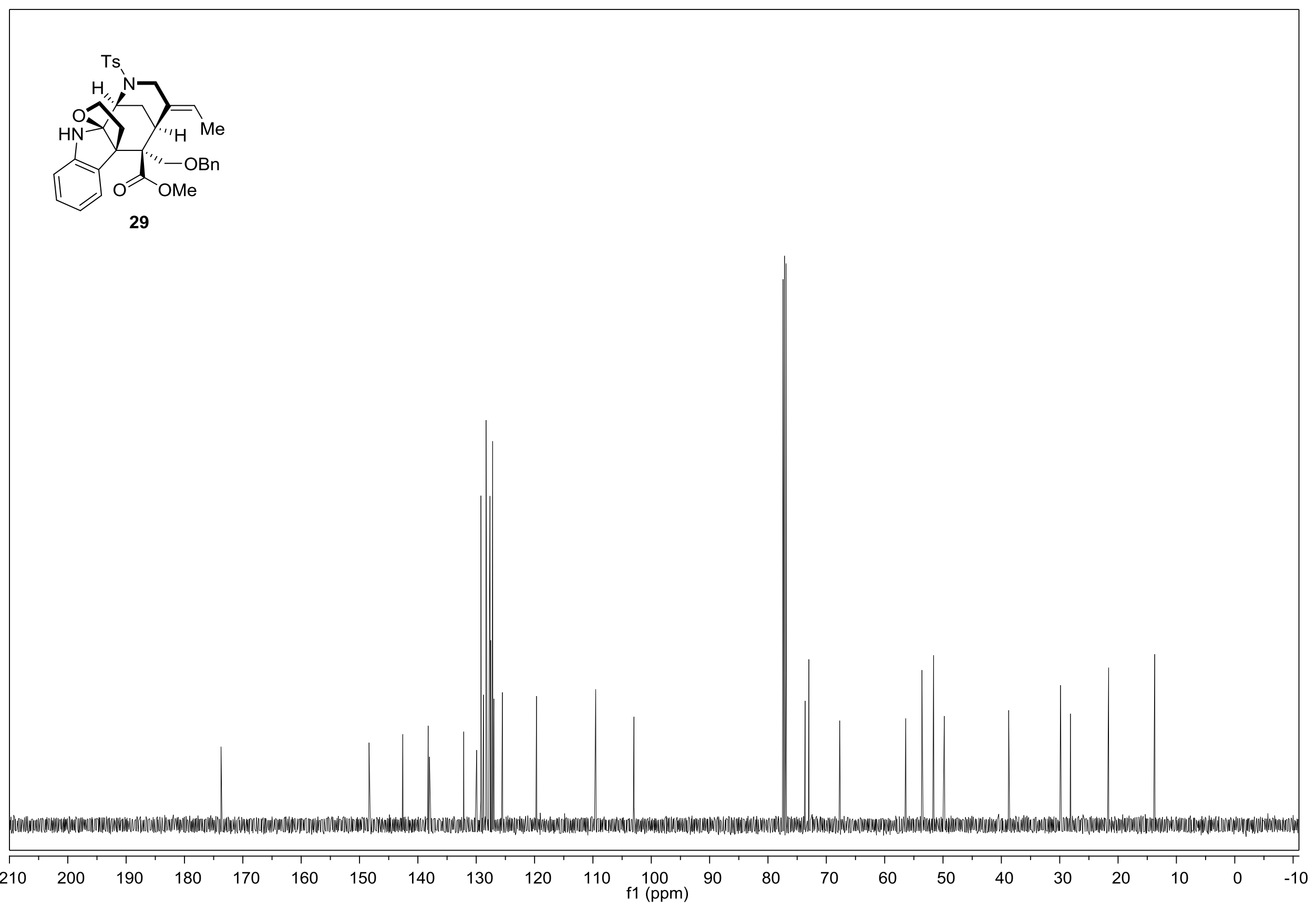


${ }^{1} \mathrm{H}$ NMR Spectrum of $4 \mathrm{a}\left(400 \mathrm{MHz}, \mathrm{CDCl}_{3}\right)$

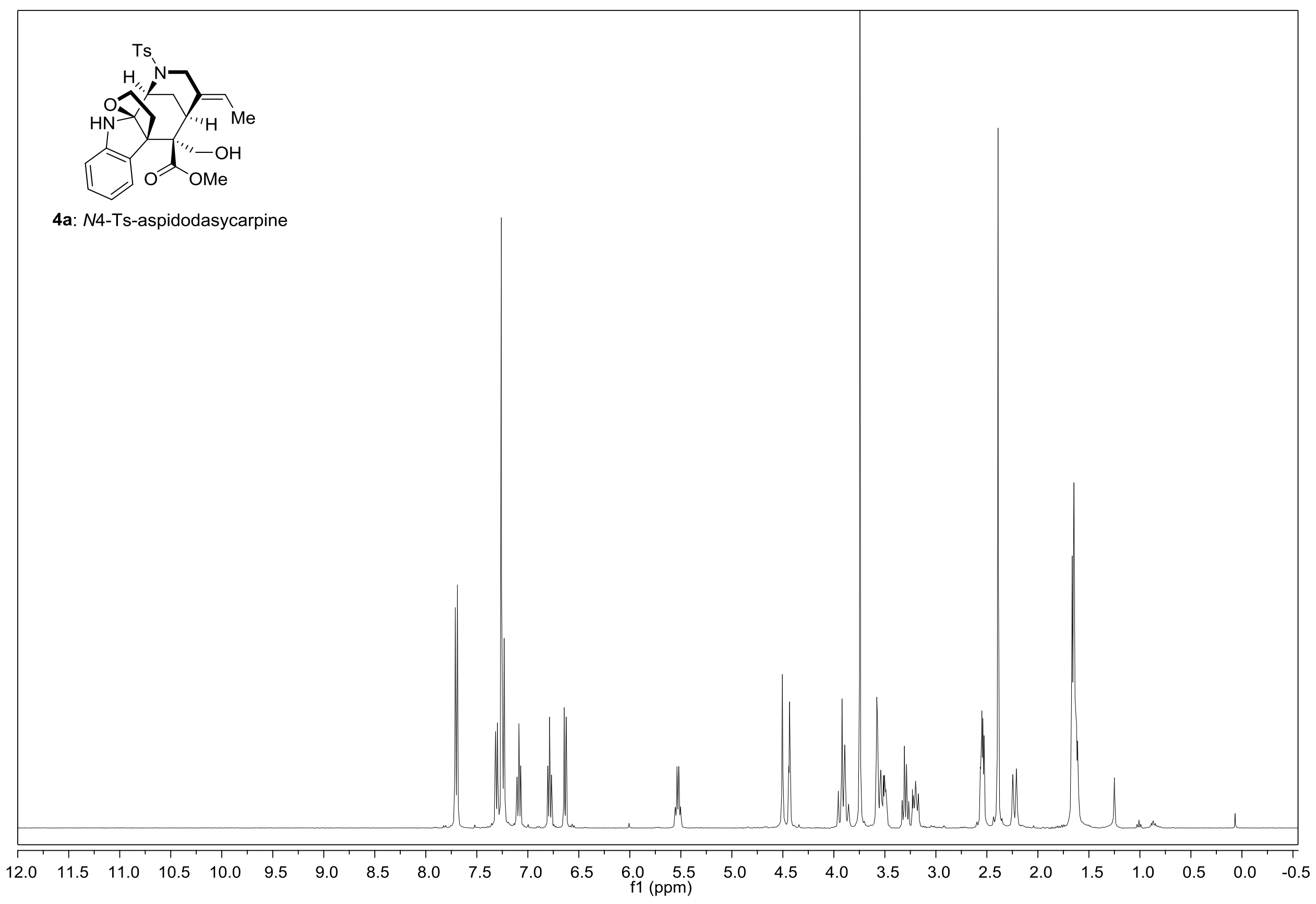




\section{${ }^{13} \mathrm{C}$ NMR Spectrum of $4 \mathrm{a}\left(126 \mathrm{MHz}, \mathrm{CDCl}_{3}\right)$}

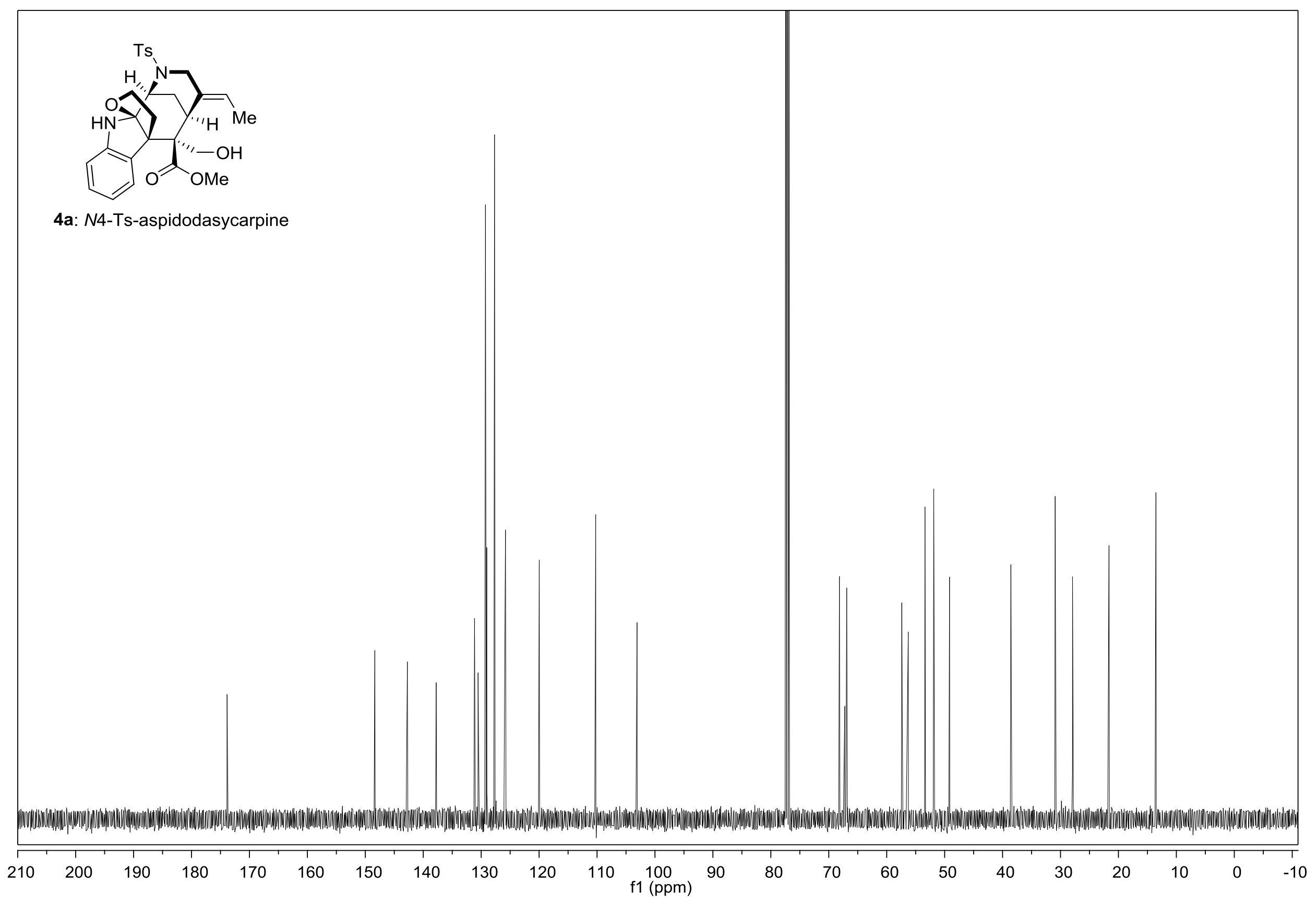




\section{${ }^{1} \mathrm{H}$ NMR Spectrum of $4\left(400 \mathrm{MHz}, \mathrm{CDCl}_{3}\right)$}

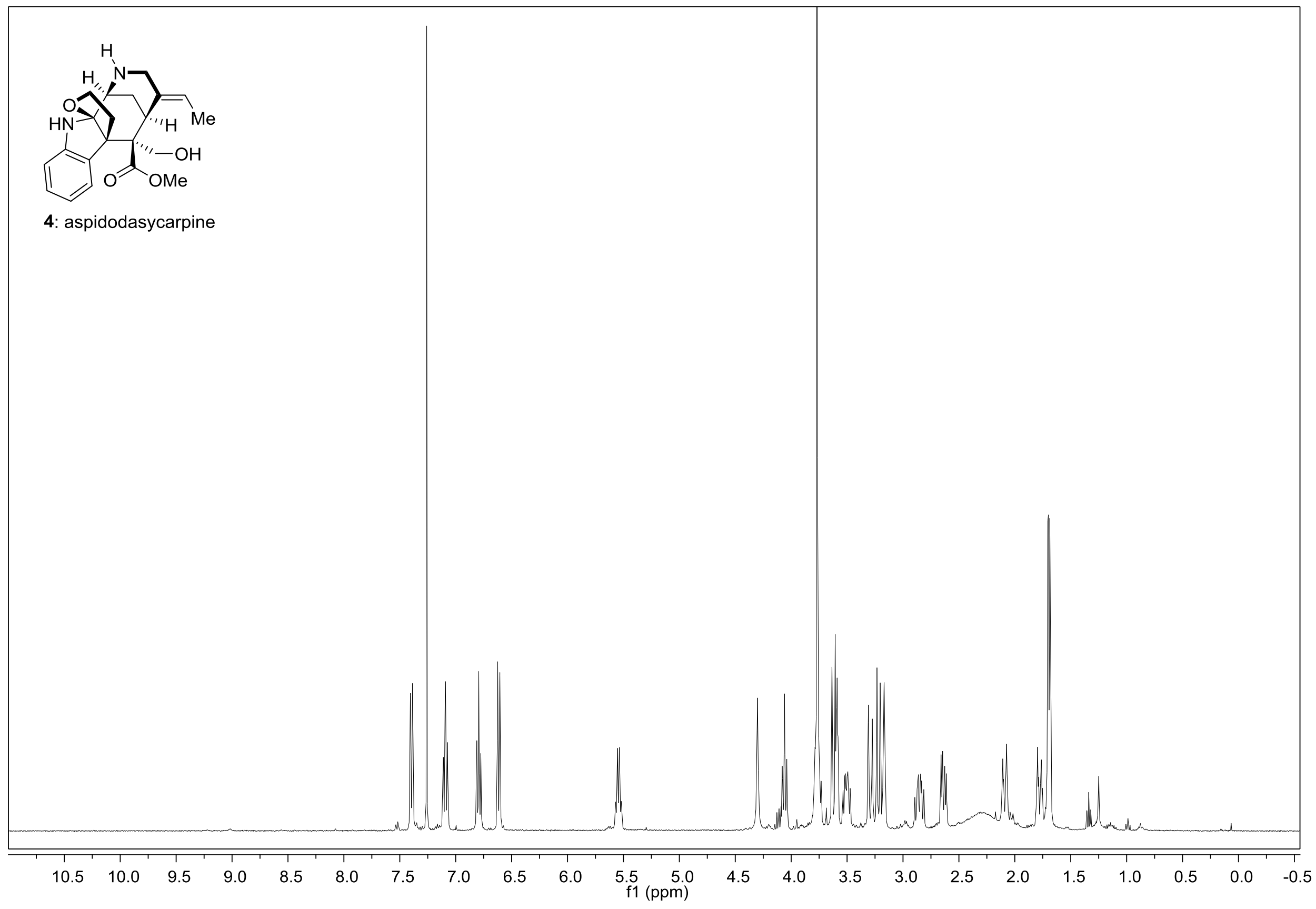


${ }^{13} \mathrm{C}$ NMR Spectrum of $4\left(101 \mathrm{MHz}, \mathrm{CDCl}_{3}\right)$

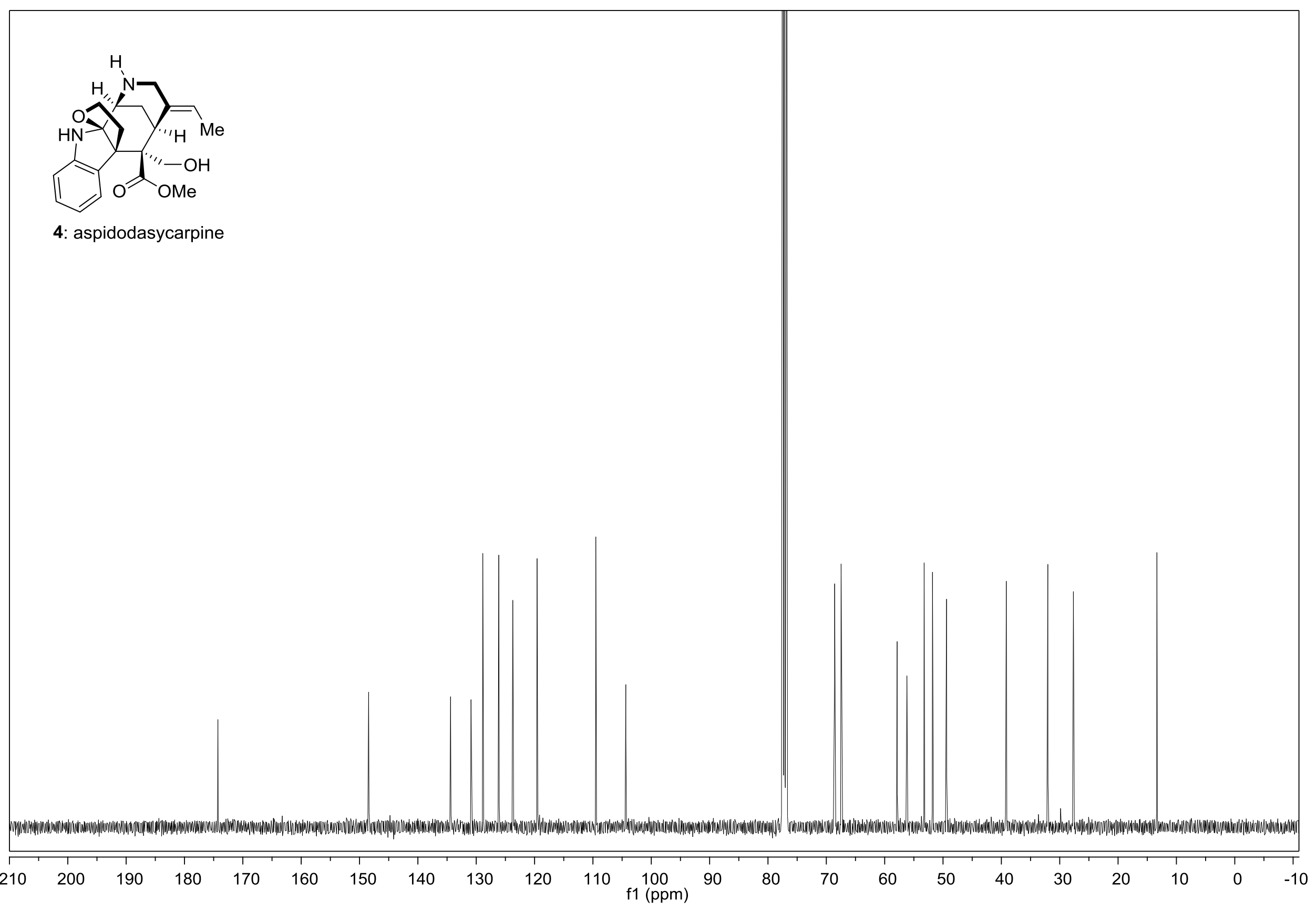




\section{${ }^{1} \mathrm{H}$ NMR Spectrum of $30\left(400 \mathrm{MHz}, \mathrm{CDCl}_{3}\right)$}

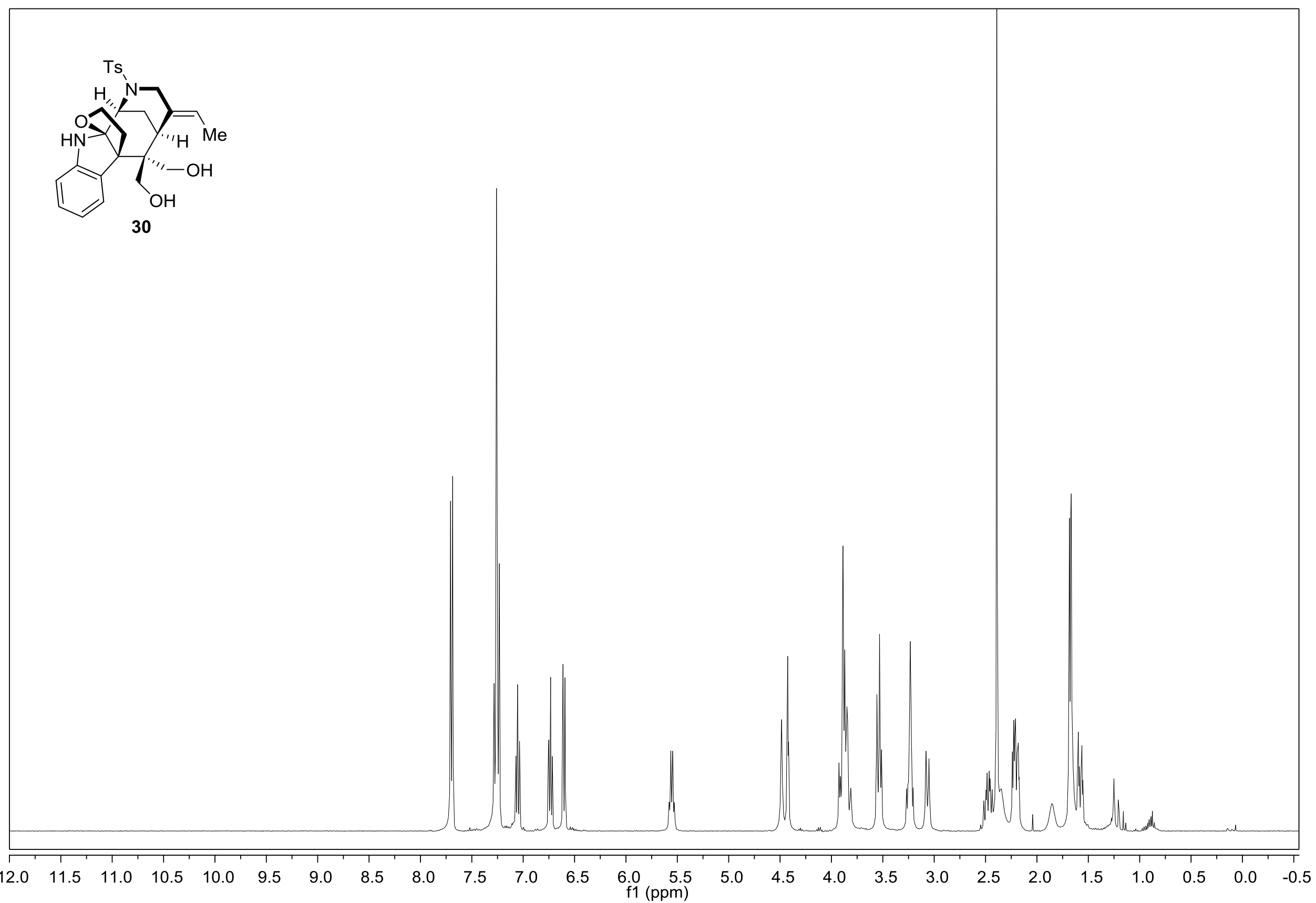

30 


\section{${ }^{13} \mathrm{C}$ NMR Spectrum of $30\left(101 \mathrm{MHz}, \mathrm{CDCl}_{3}\right)$}

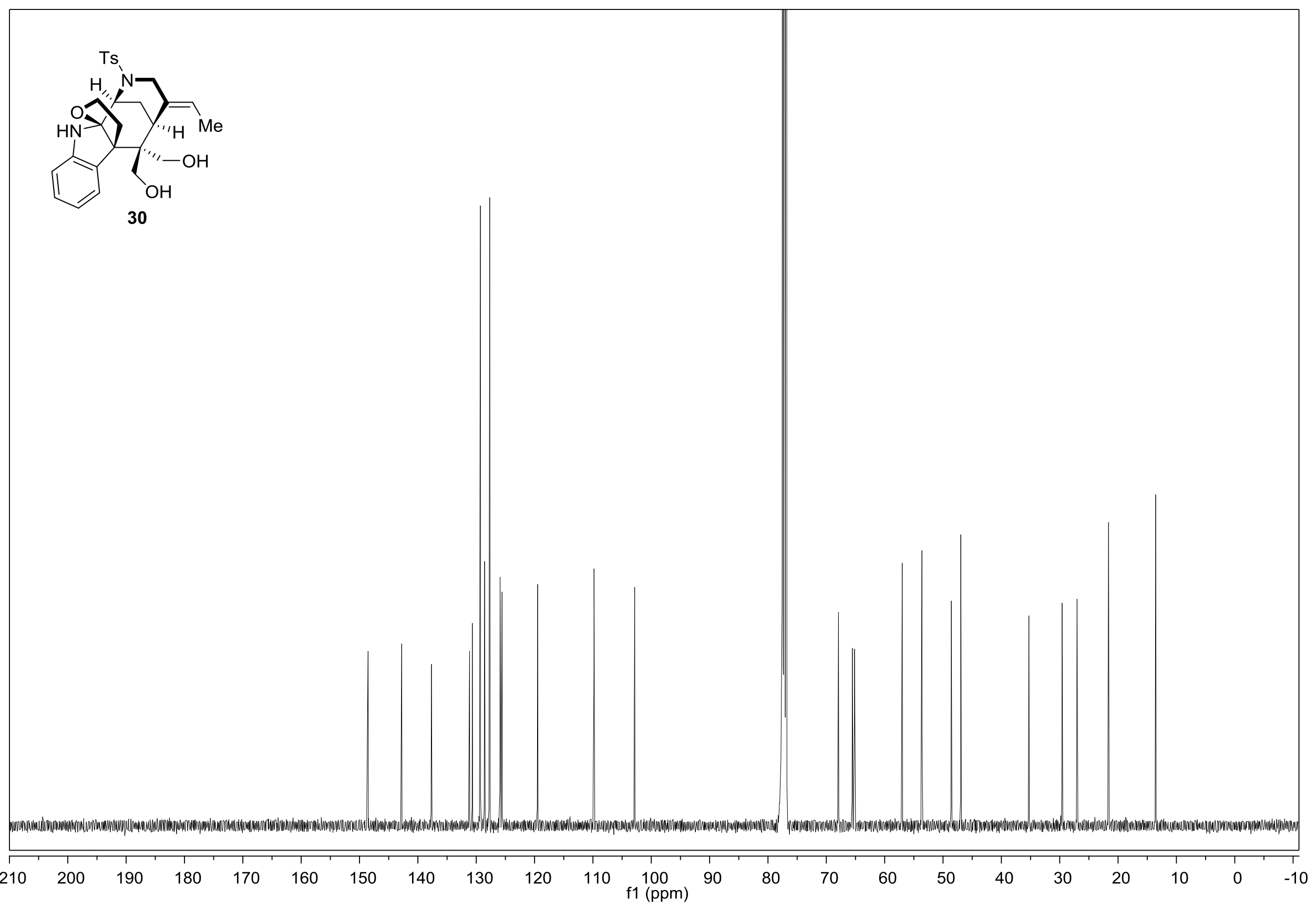




\section{${ }^{1} \mathrm{H}$ NMR Spectrum of $5 \mathrm{a}\left(400 \mathrm{MHz}, \mathrm{CDCl}_{3}\right)$}

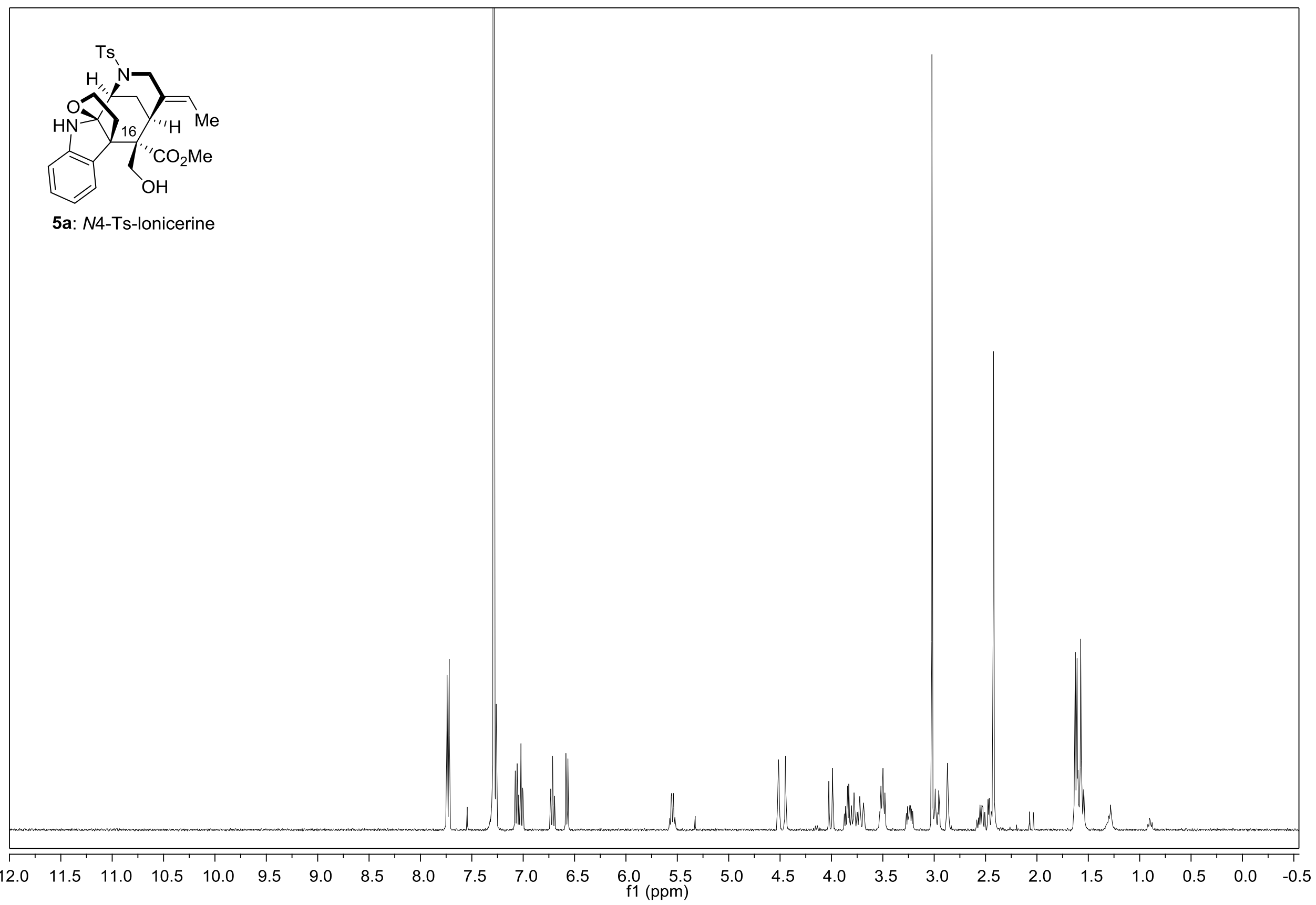




\section{${ }^{13} \mathrm{C}$ NMR Spectrum of $5 \mathrm{a}\left(101 \mathrm{MHz}, \mathrm{CDCl}_{3}\right)$}

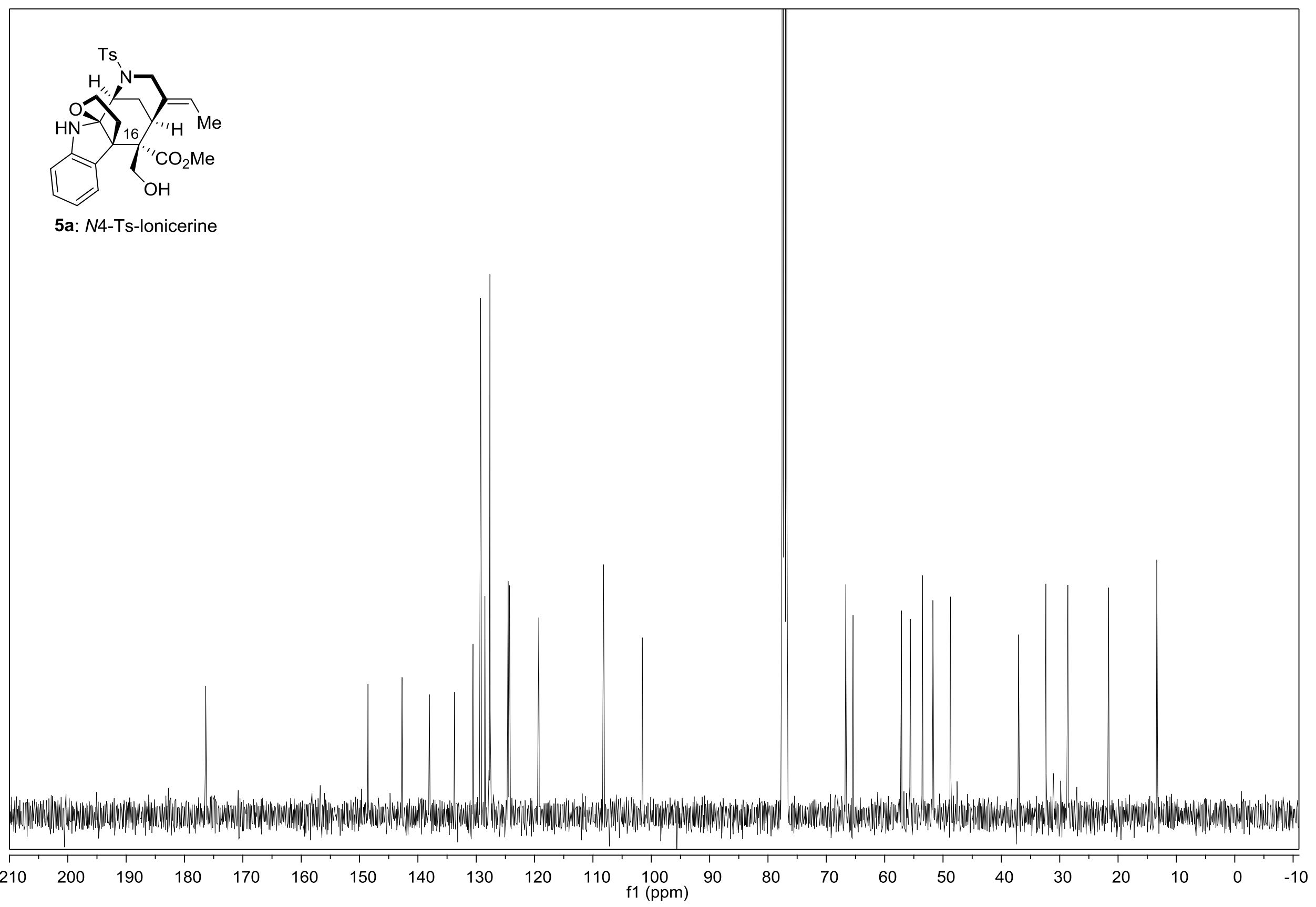


${ }^{1} \mathrm{H}$ NMR Spectrum of 5 (500 $\left.\mathrm{MHz}, \mathrm{CDCl}_{3}\right)$

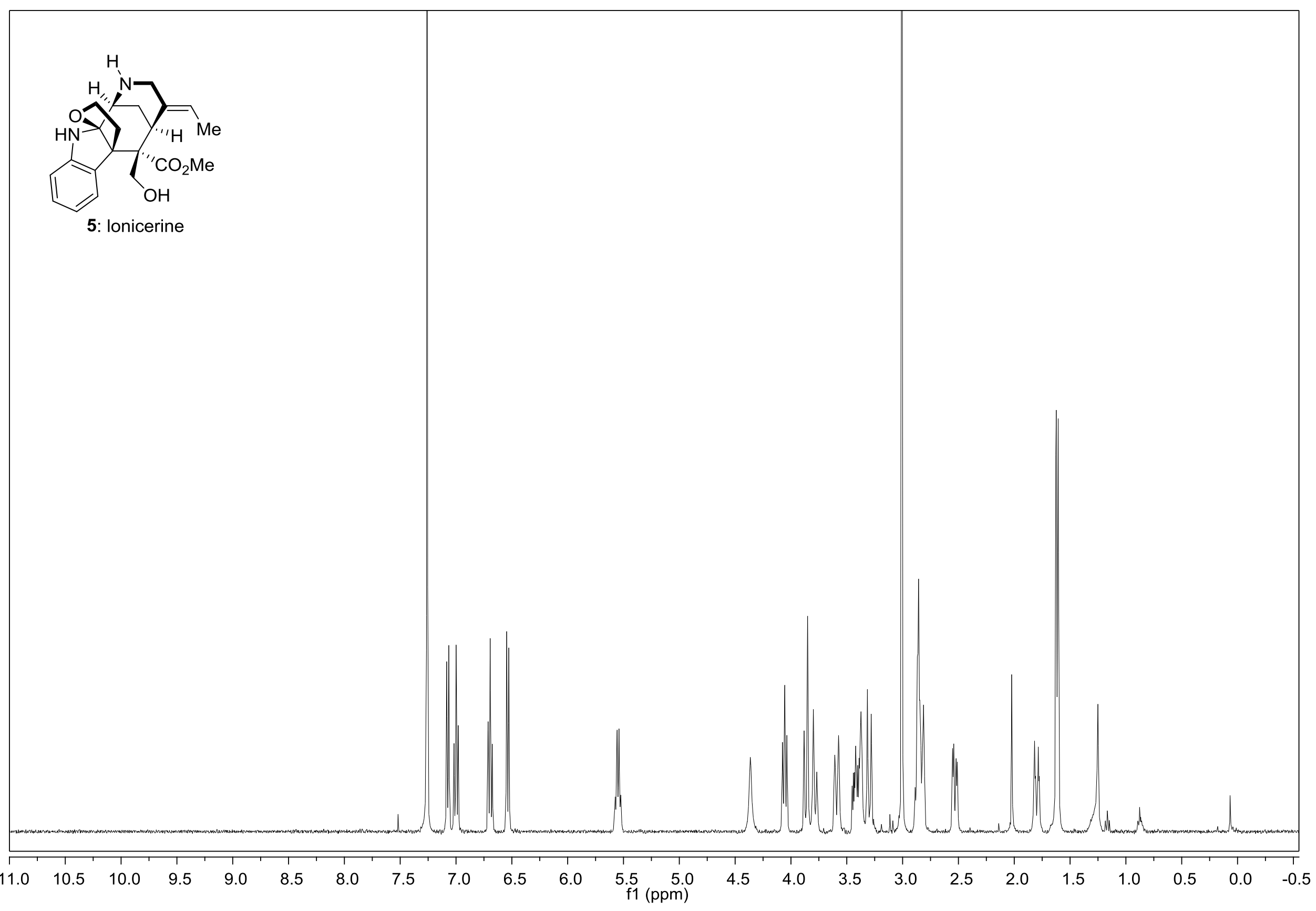


${ }^{13} \mathrm{C}$ NMR Spectrum of $5\left(126 \mathrm{MHz}, \mathrm{CDCl}_{3}\right)$

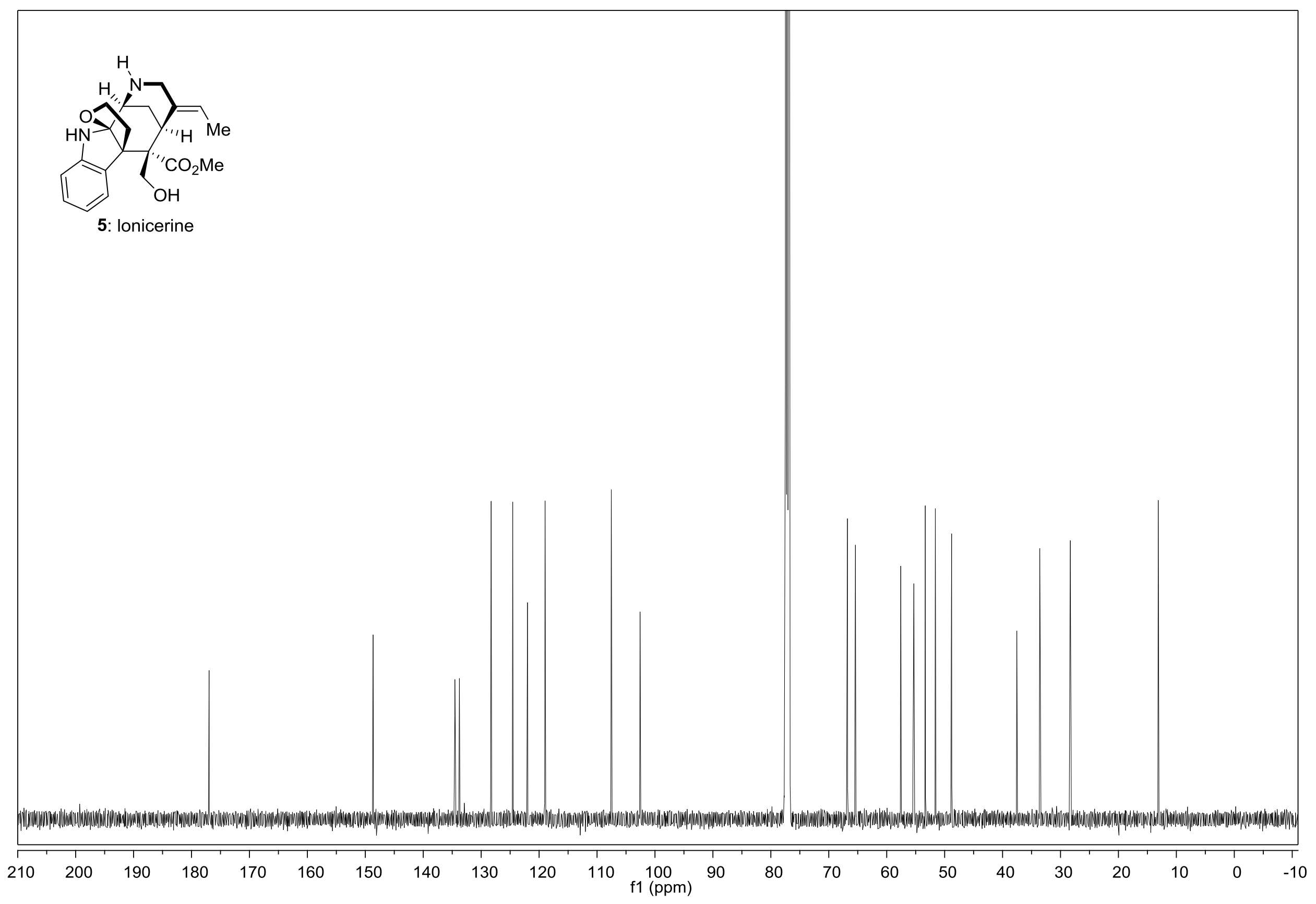




\section{${ }^{1} \mathrm{H}$ NMR Spectrum of $31\left(400 \mathrm{MHz}, \mathrm{CDCl}_{3}\right)$}

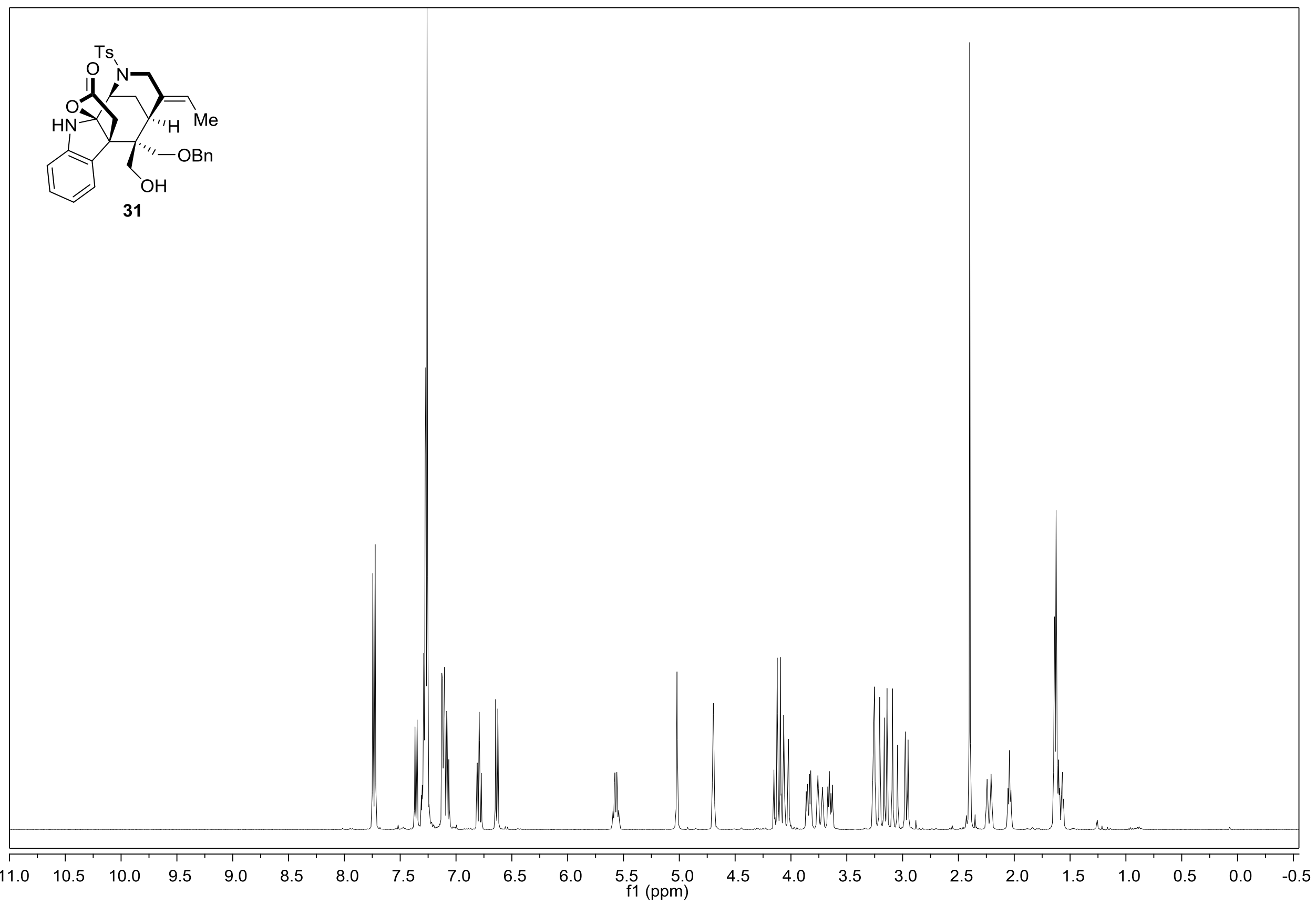




\section{${ }^{13} \mathrm{C}$ NMR Spectrum of $31\left(101 \mathrm{MHz}, \mathrm{CDCl}_{3}\right)$}

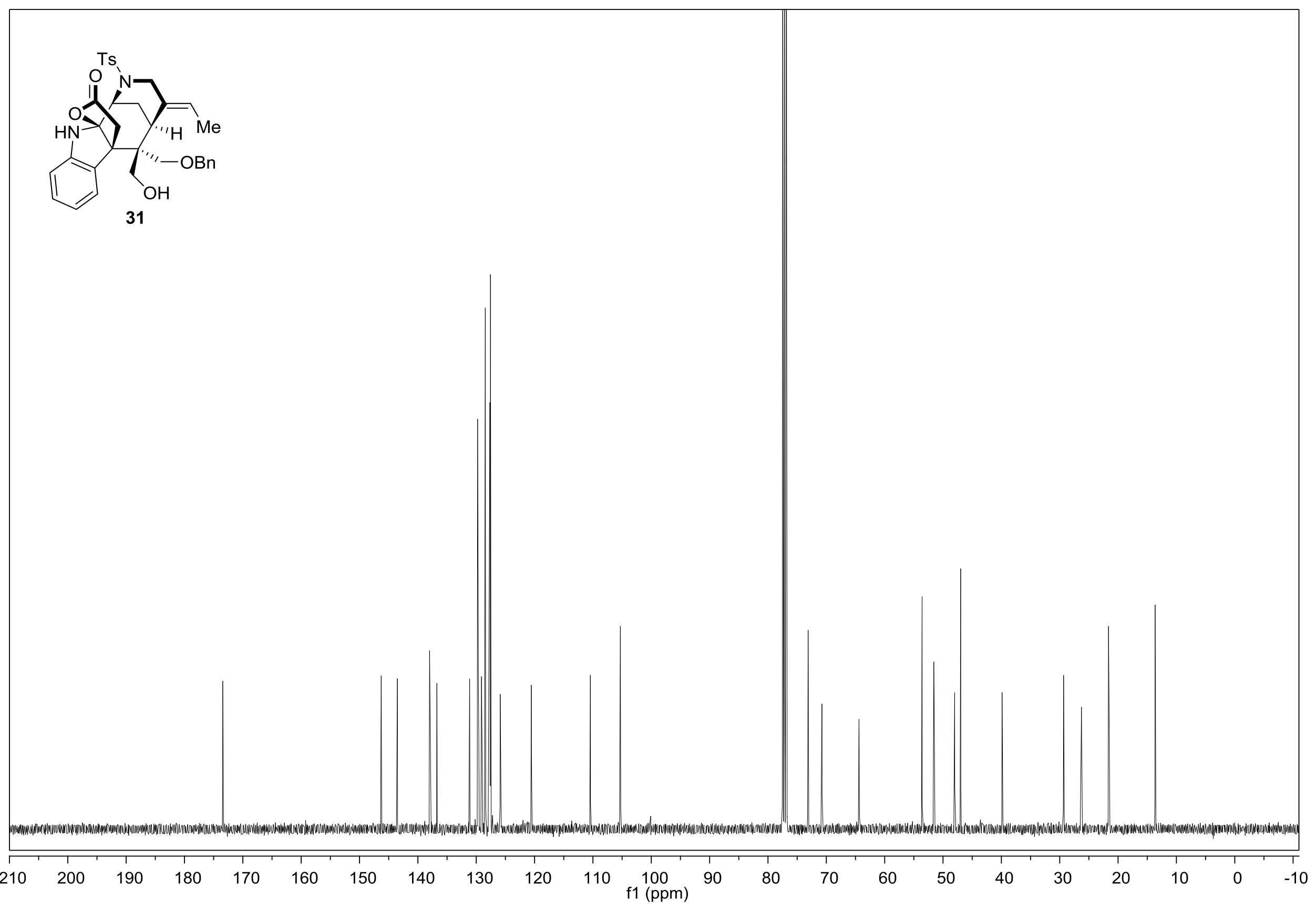


${ }^{1} \mathrm{H}$ NMR Spectrum of $33\left(400 \mathrm{MHz}, \mathrm{CDCl}_{3}\right)$

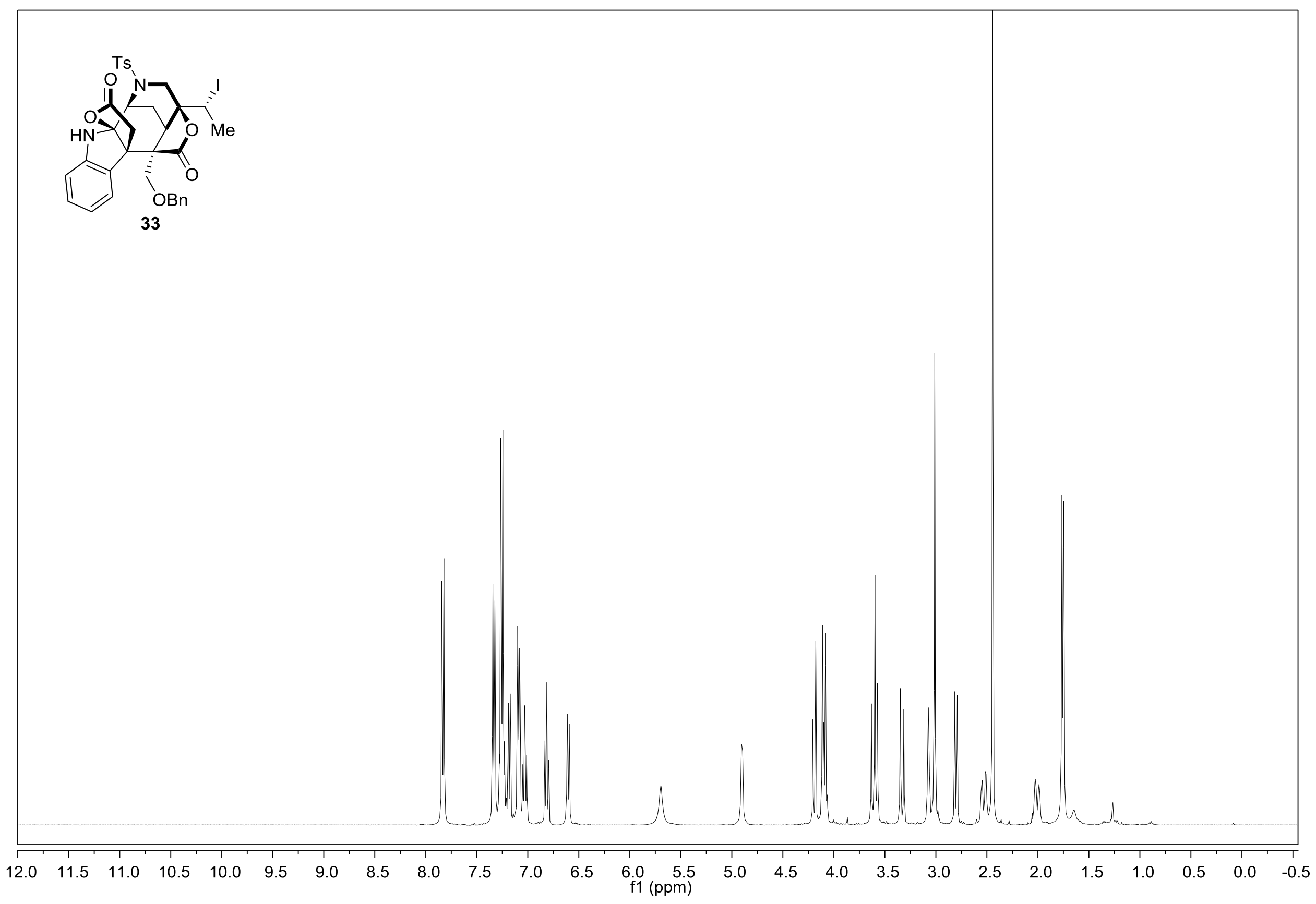


${ }^{13} \mathrm{C}$ NMR Spectrum of $33\left(101 \mathrm{MHz}, \mathrm{CDCl}_{3}\right)$

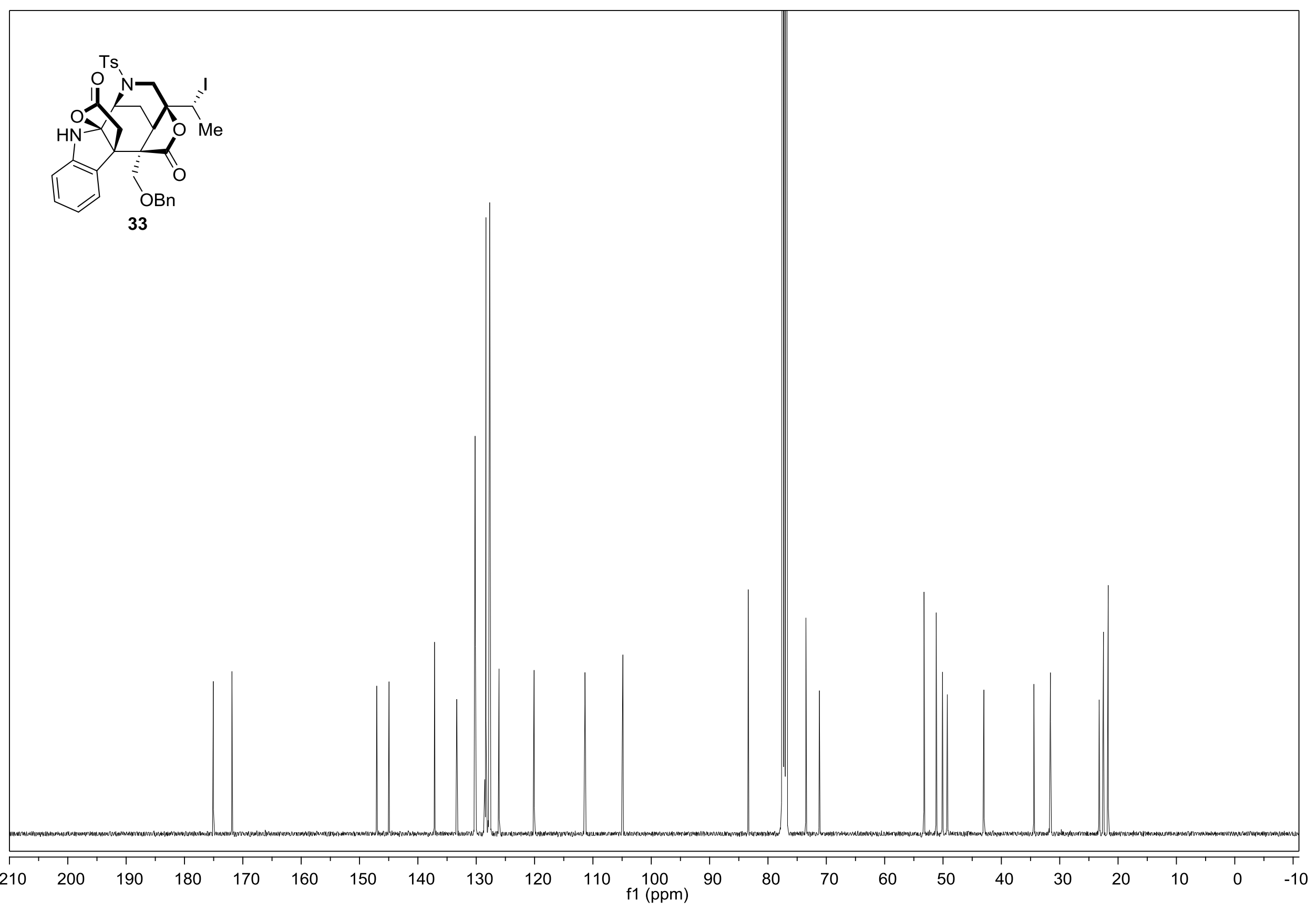




\section{${ }^{1} \mathrm{H}$ NMR Spectrum of $32\left(400 \mathrm{MHz}, \mathrm{CDCl}_{3}\right)$}

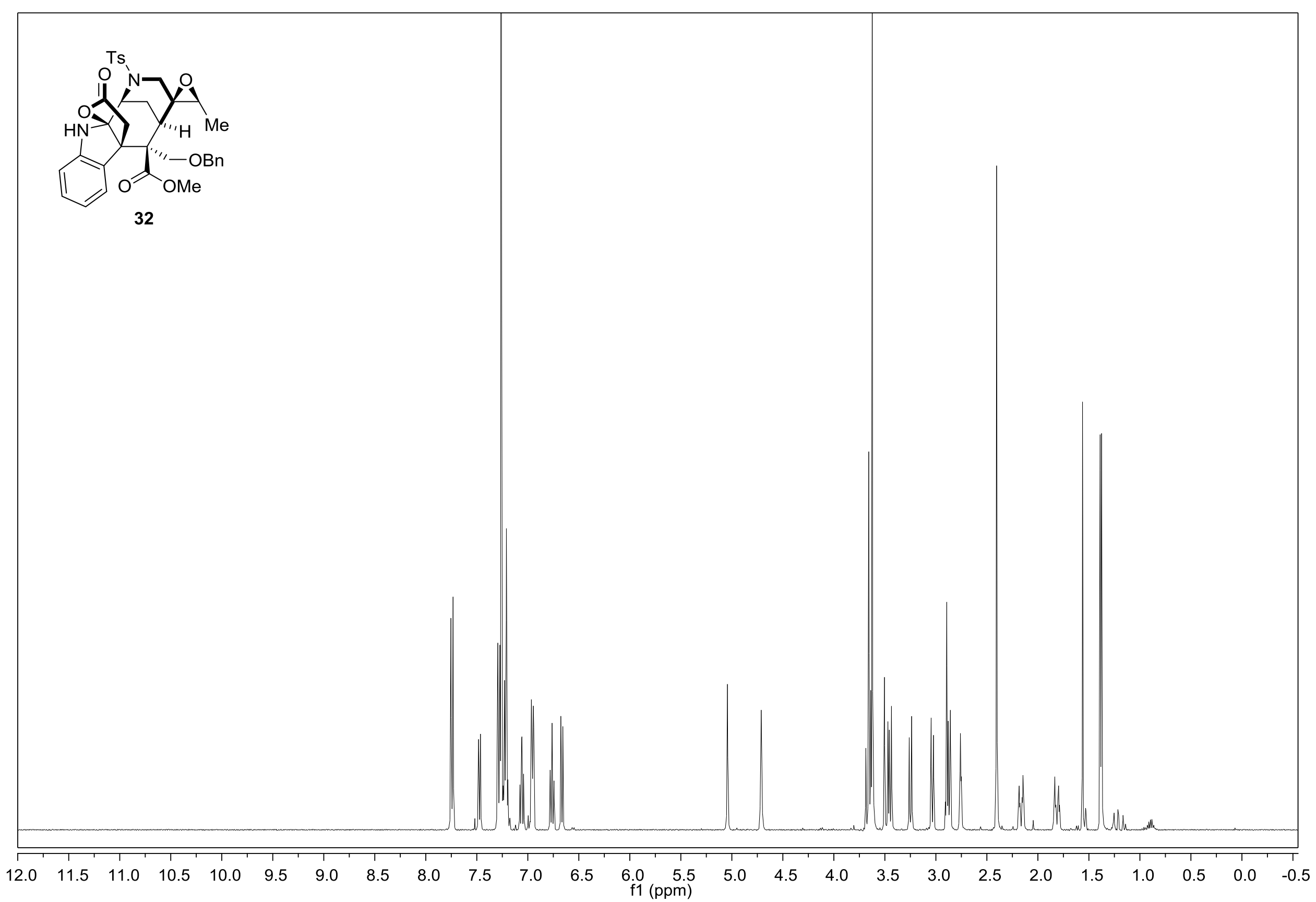

32 


\section{${ }^{13} \mathrm{C}$ NMR Spectrum of $32\left(101 \mathrm{MHz}, \mathrm{CDCl}_{3}\right)$}

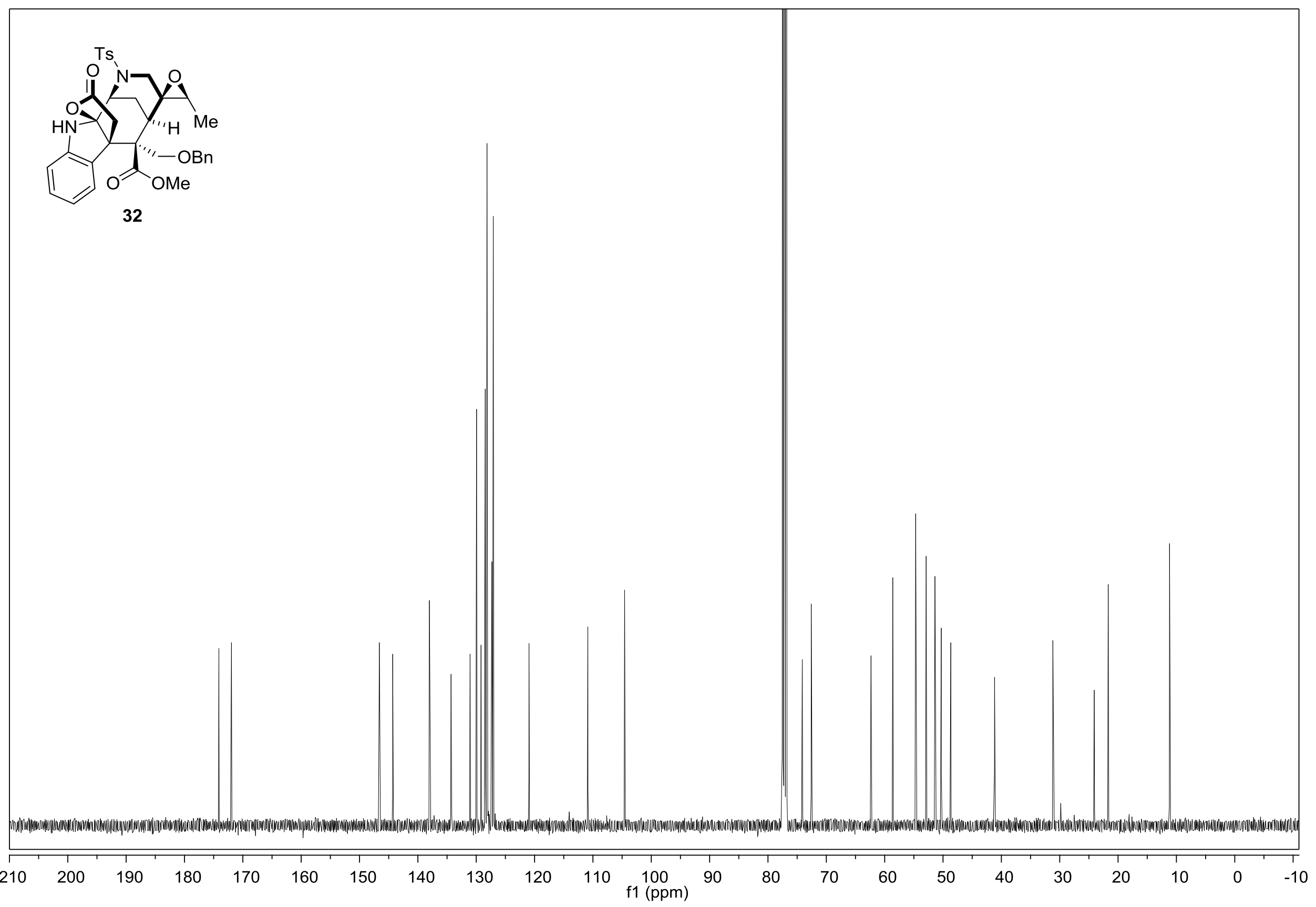


${ }^{1} \mathrm{H}$ NMR Spectrum of $34\left(400 \mathrm{MHz}, \mathrm{CDCl}_{3}\right)$

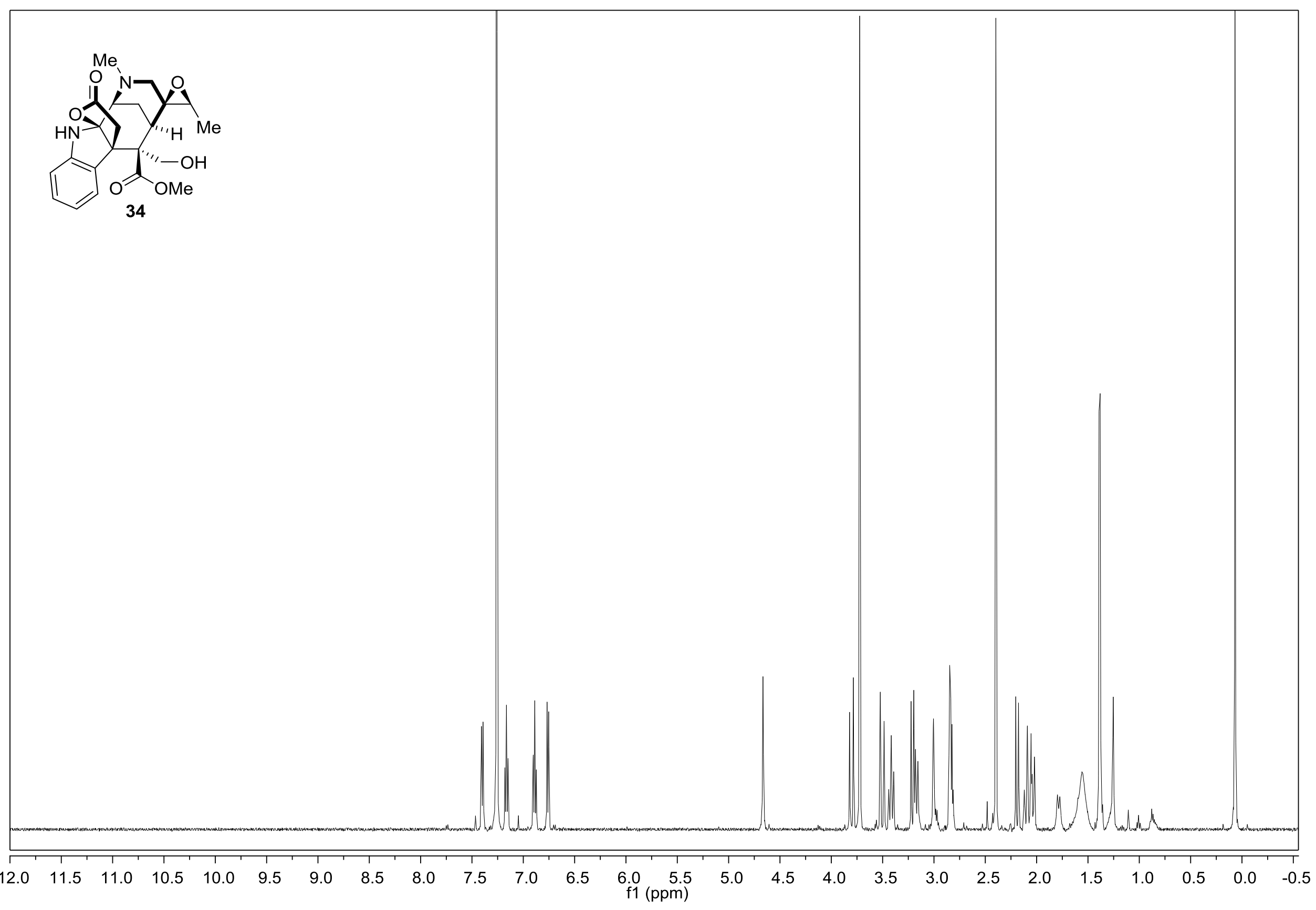


${ }^{13} \mathrm{C}$ NMR Spectrum of 34 (126 $\left.\mathrm{MHz}, \mathrm{CDCl}_{3}\right)$

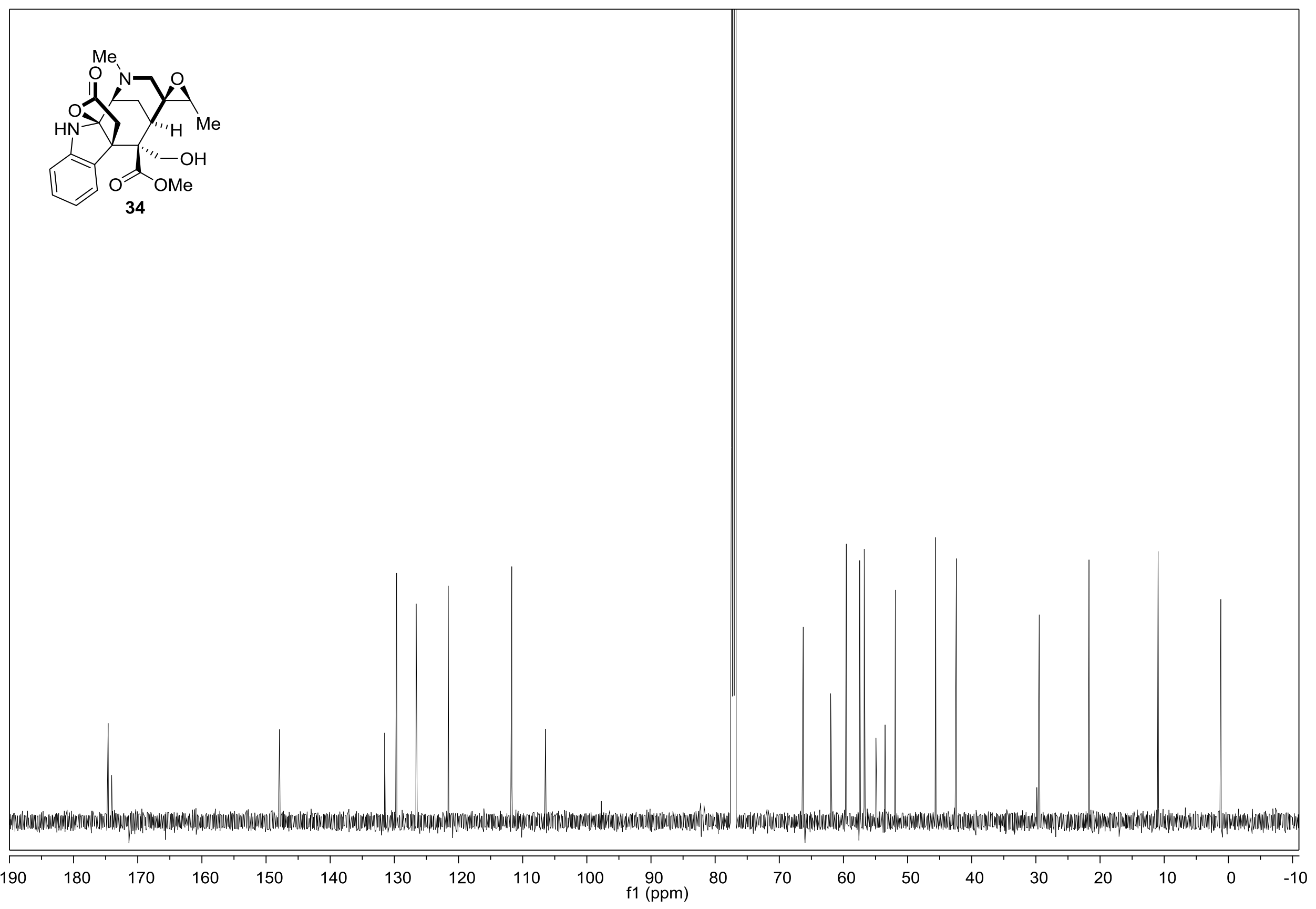


${ }^{1} \mathrm{H}$ NMR Spectrum of $6\left(600 \mathrm{MHz}, \mathrm{CDCl}_{3}\right)$

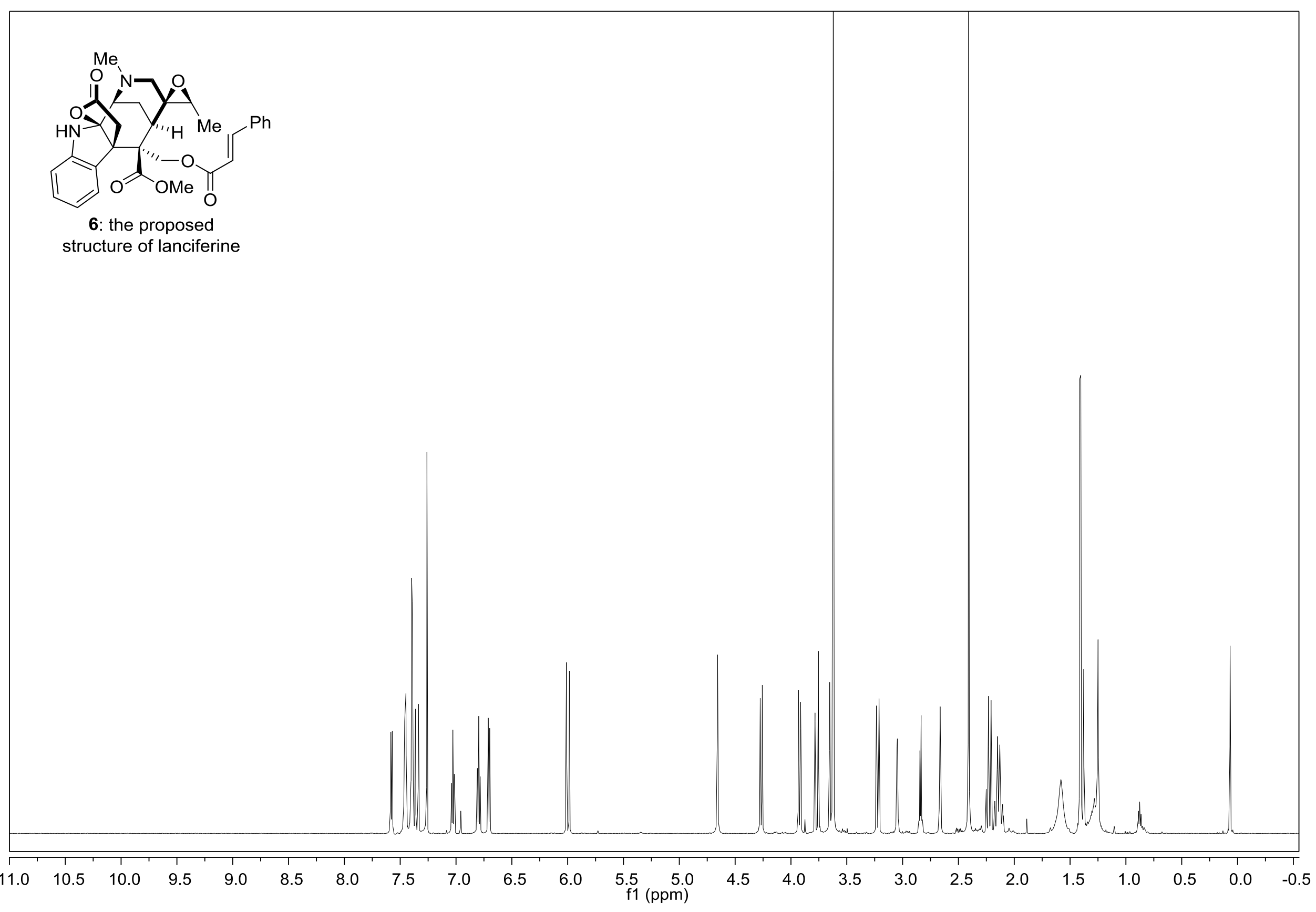


${ }^{13} \mathrm{C}$ NMR Spectrum of $6\left(151 \mathrm{MHz}, \mathrm{CDCl}_{3}\right)$

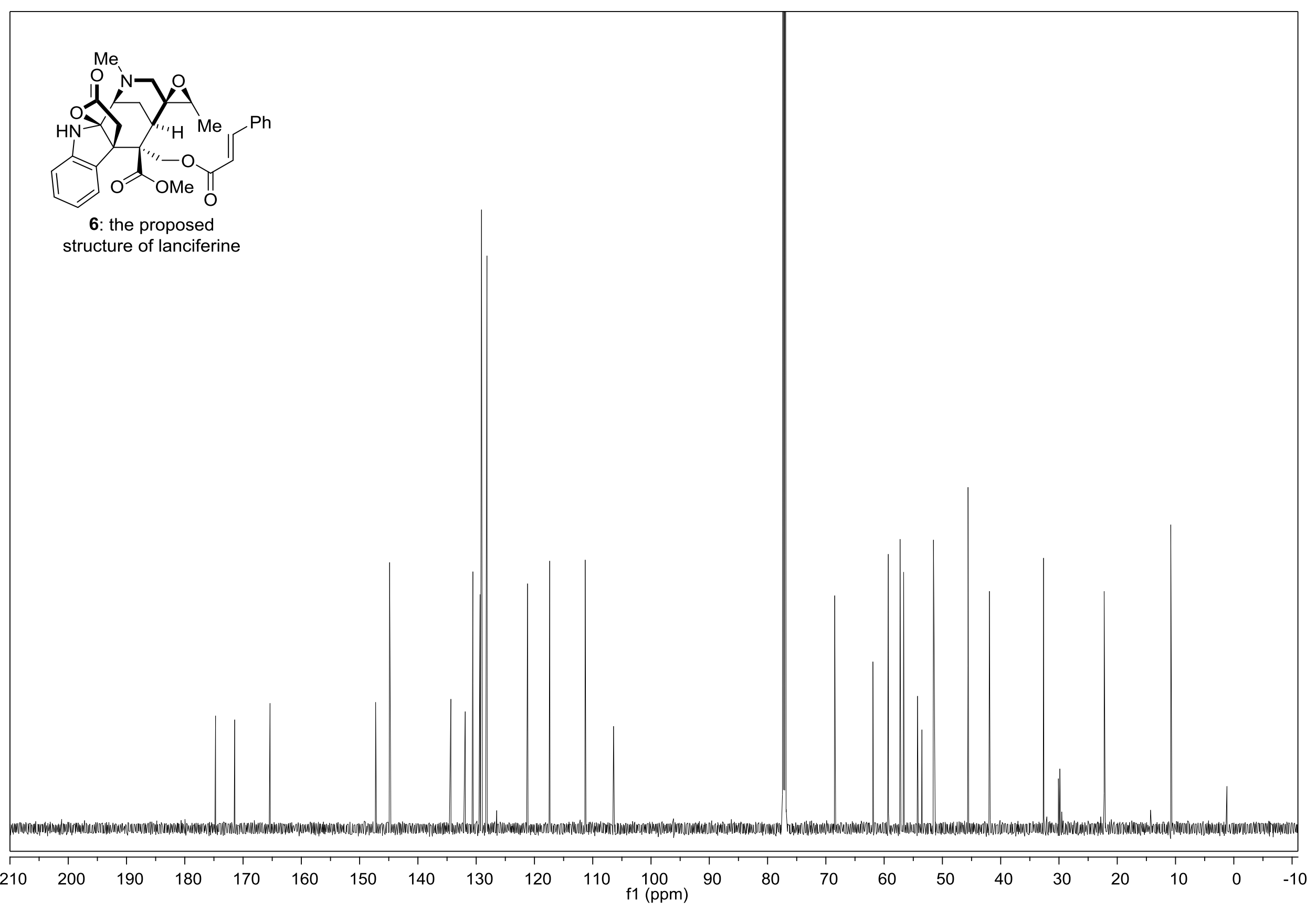


V Comparison of the NMR Spectra and Data of Natural and Synthetic Aspidodasycarpine and Lonicerine

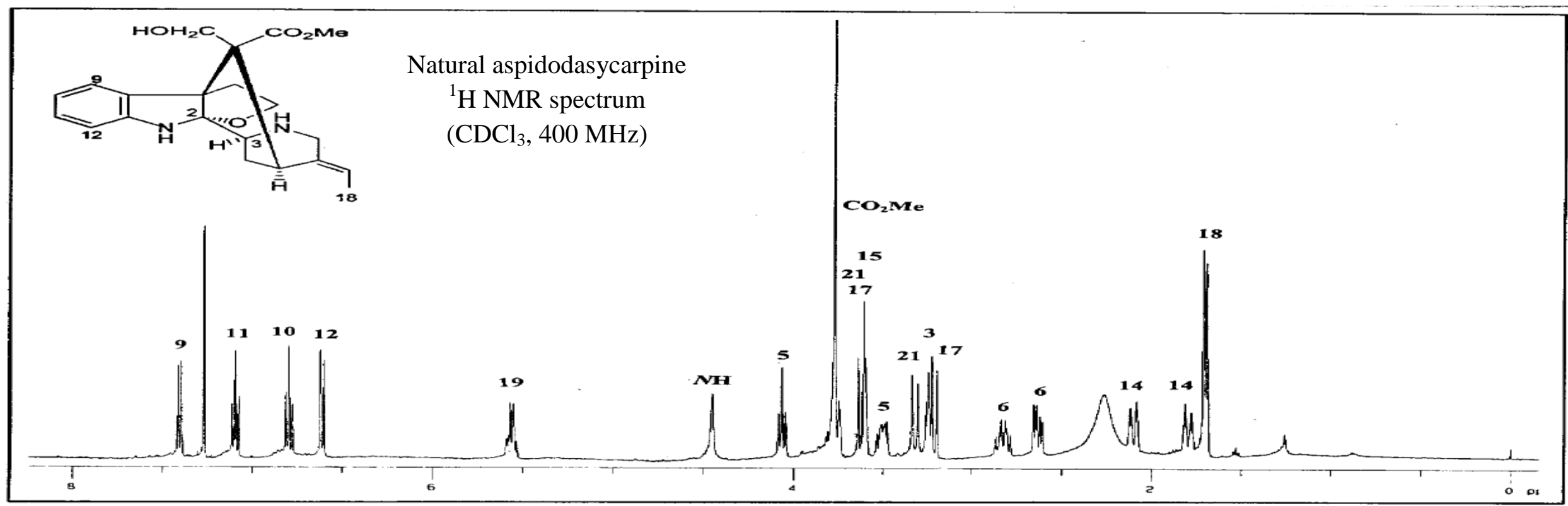

Fig. 2.88 : ${ }^{1} \mathrm{H}$ NMR Spectrum (CDCl 3 , $400 \mathrm{MHz}$ ) of Aspidodasycarpine 71

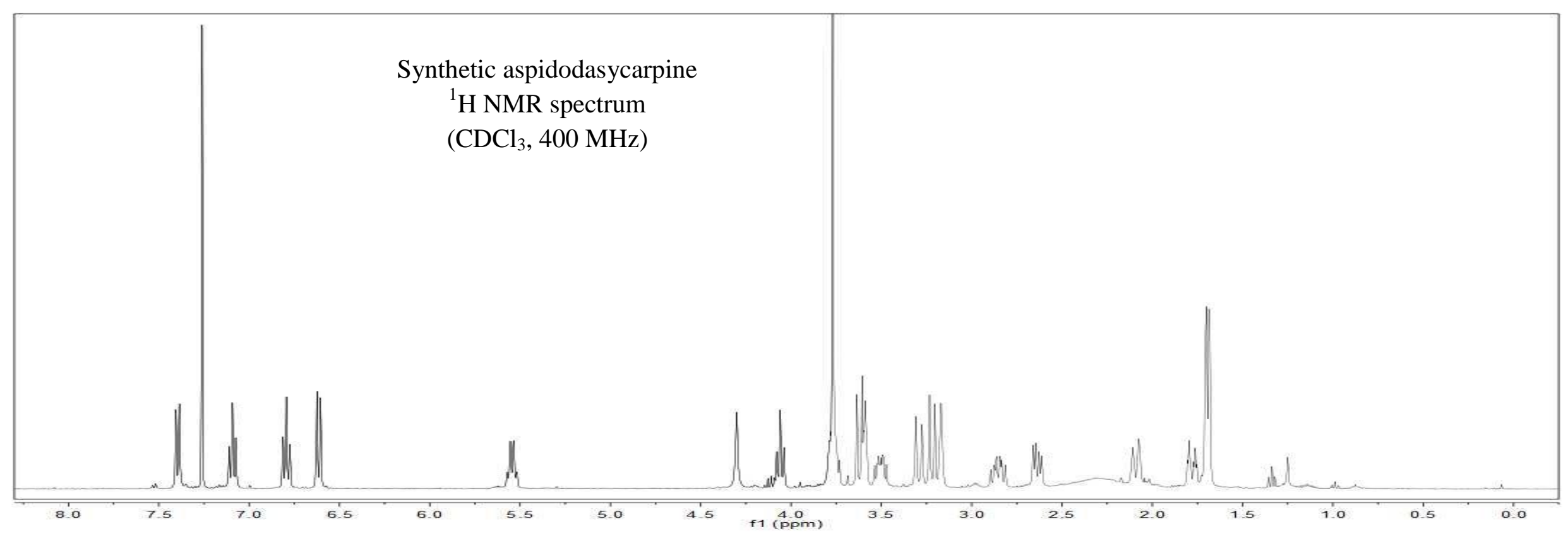



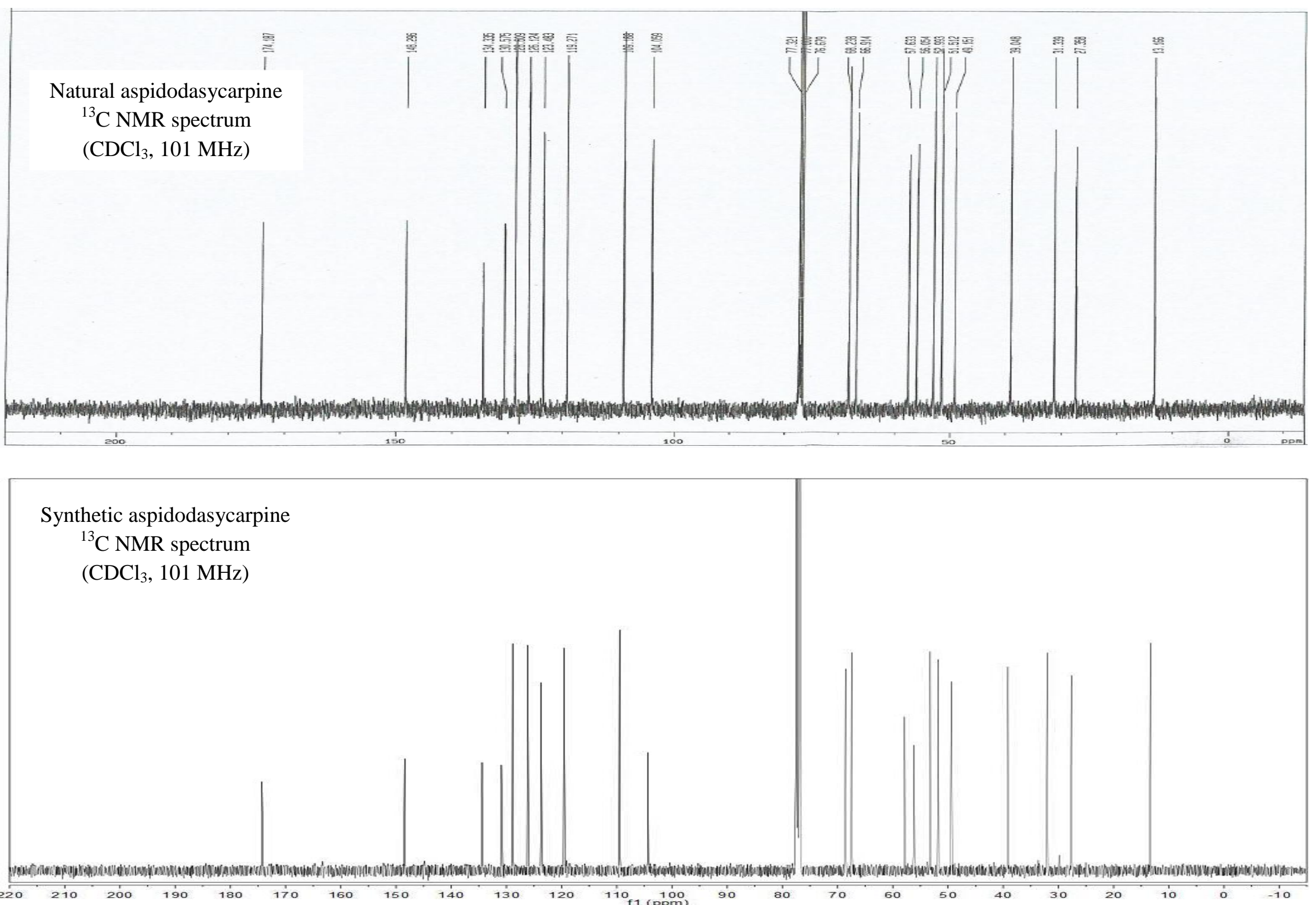

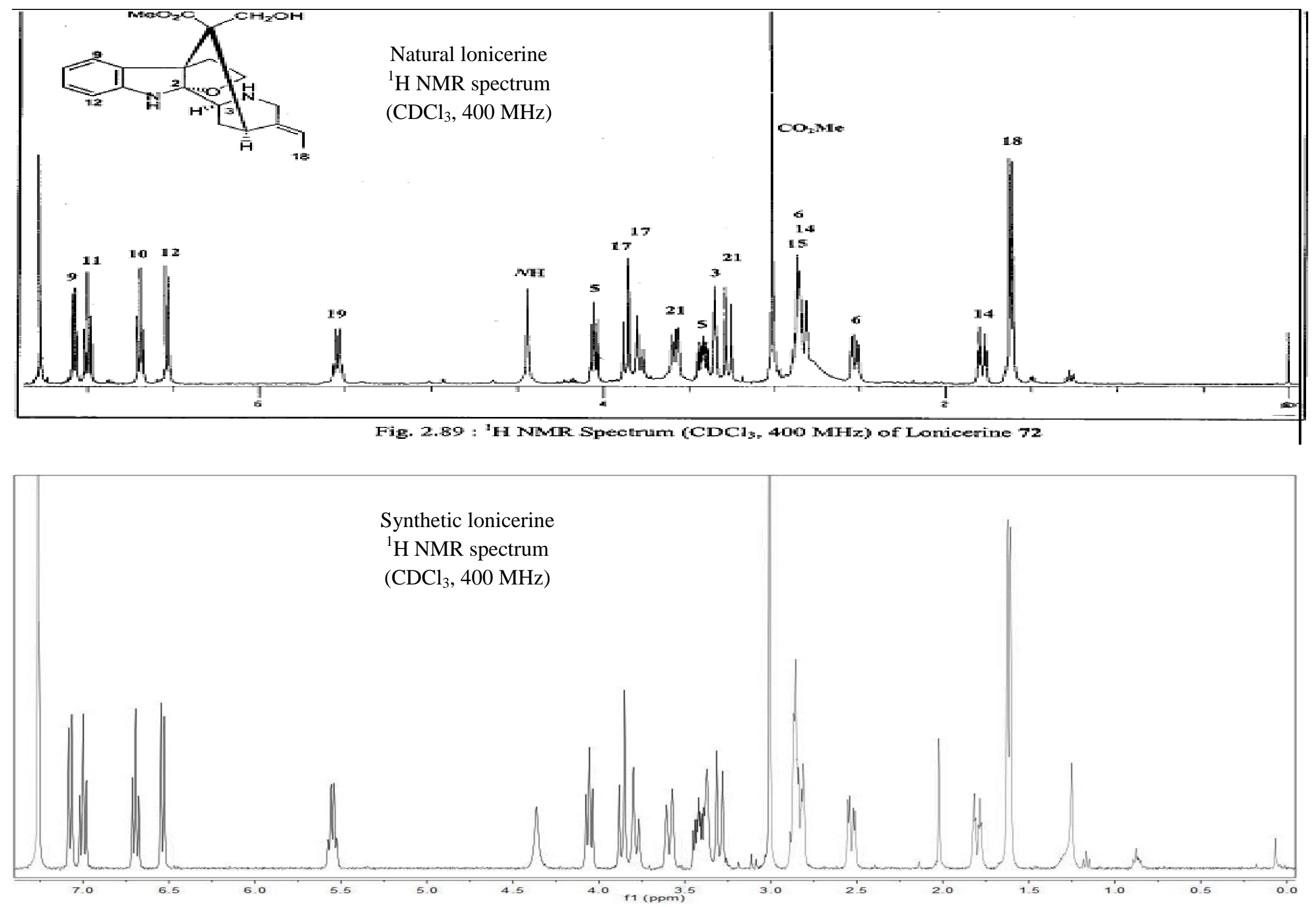

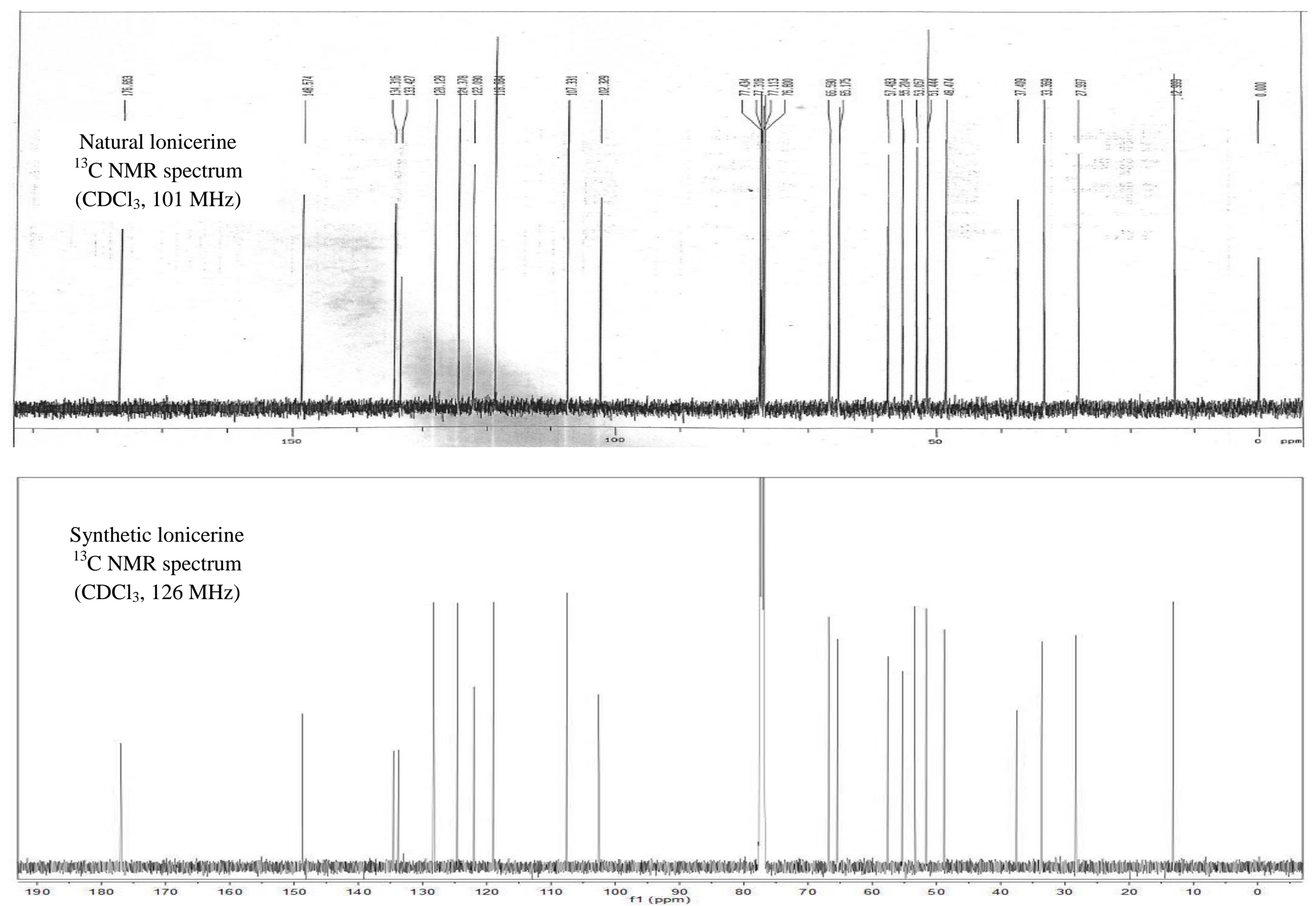
Table S1. Comparison of the ${ }^{1} \mathrm{H}$ NMR $\left(\mathrm{CDCl}_{3}\right)$ data of natural and synthetic aspidodasycapine

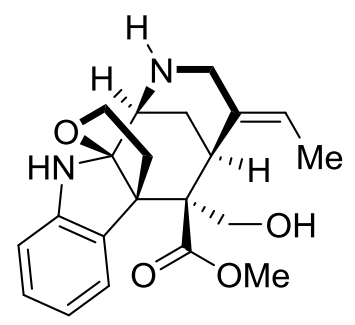

4: aspidodasycarpine

\begin{tabular}{|c|c|c|c|c|}
\hline \multicolumn{2}{|c|}{$\begin{array}{c}\text { Natural } \\
\delta_{\mathrm{H}}[\mathrm{ppm}, \text { mult, } J(\mathrm{~Hz})] \\
600 \mathrm{MHz}\end{array}$} & \multicolumn{2}{|c|}{$\begin{array}{c}\text { Synthetic } \\
\delta_{\mathrm{H}}[\mathrm{ppm}, \text { mult, } J(\mathrm{~Hz})] \\
400 \mathrm{MHz}\end{array}$} & \multirow{2}{*}{$\begin{array}{c}\text { Err } \\
\begin{array}{c}\text { (Natural-Synthetic) } \\
\Delta \delta_{\mathrm{H}}(\mathrm{ppm})\end{array} \\
0\end{array}$} \\
\hline 7.4 & $1 \mathrm{H}, \mathrm{d}, 7.5$ & 7.40 & $1 \mathrm{H}, \mathrm{d}, 7.5$ & \\
\hline 7.09 & $1 \mathrm{H}, \mathrm{t}, 7.5$ & 7.09 & $1 \mathrm{H}, \mathrm{td}, 7.7,1.0$ & - \\
\hline 6.8 & $1 \mathrm{H}, \mathrm{t}, 7.5$ & 6.79 & $1 \mathrm{H}, \mathrm{t}, 7.4$ & 0.01 \\
\hline 6.61 & $1 \mathrm{H}, \mathrm{d}, 7.5$ & 6.61 & $1 \mathrm{H}, \mathrm{d}, 7.8$ & 0 \\
\hline 5.56 & $1 \mathrm{H}, \mathrm{q}, 7$ & 5.54 & $1 \mathrm{H}, \mathrm{q}, 6.9$ & 0.02 \\
\hline 4.45 & $1 \mathrm{H}(N 4 \mathbf{H}), \mathrm{s}$ & 4.30 & $1 \mathrm{H}(N 4 \mathbf{H}), \mathrm{s}$ & 0.15 \\
\hline 4.07 & $1 \mathrm{H}, \mathrm{t}, 8.0$ & 4.06 & $1 \mathrm{H}, \mathrm{t}, 8.1$ & 0.01 \\
\hline 3.78 & $3 \mathrm{H}, \mathrm{s}$ & 3.77 & $3 \mathrm{H}, \mathrm{s}$ & 0.01 \\
\hline 3.74 & $1 \mathrm{H}, \mathrm{d}, 14$ & $3.81-3.72$ & $1 \mathrm{H}$, overlapped & - \\
\hline 3.63 & $1 \mathrm{H}, \mathrm{d}, 11.5$ & 3.62 & $1 \mathrm{H}, \mathrm{d}, 11.6$ & 0.01 \\
\hline 3.62 & $1 \mathrm{H}, \mathrm{m}$ & 3.59 & $1 \mathrm{H}, \mathrm{dd}, 3.0,3.0$ & - \\
\hline 3.51 & $1 \mathrm{H}, \mathrm{m}$ & 3.50 & $1 \mathrm{H}, \mathrm{ddd}, 11.3,8.4,5.9$ & - \\
\hline 3.32 & $1 \mathrm{H}, \mathrm{d}, 14$ & 3.29 & $1 \mathrm{H}, \mathrm{d}, 13.9$ & 0.03 \\
\hline 3.21 & $1 \mathrm{H}, \mathrm{d}, 11.5$ & 3.22 & $1 \mathrm{H}, \mathrm{d}, 11.5$ & -0.01 \\
\hline 3.26 & $1 \mathrm{H}, \mathrm{br}, \mathrm{s}$ & 3.17 & $1 \mathrm{H}, \mathrm{dd}, 2.5,2.5$ & - \\
\hline 2.83 & $1 \mathrm{H}, \mathrm{td}, 13,8$ & $2.92-2.79$ & $1 \mathrm{H}, \mathrm{m}$ & - \\
\hline 2.63 & $1 \mathrm{H}, \mathrm{dd}, 13,5.5$ & 2.64 & $1 \mathrm{H}, \mathrm{dd}, 13.1,5.7$ & -0.01 \\
\hline 2.1 & $1 \mathrm{H}, \mathrm{d}, 14$ & 2.09 & $1 \mathrm{H}, \mathrm{d}, 13.6$ & 0.01 \\
\hline 1.79 & $1 \mathrm{H}, \mathrm{dt}, 14,3.4$ & 1.78 & $1 \mathrm{H}, \mathrm{dt}, 13.8,3.4$ & 0.01 \\
\hline 1.71 & $3 \mathrm{H}, \mathrm{d}, 7$ & 1.69 & $3 \mathrm{H}, \mathrm{dd}, 6.9,1.9$ & - \\
\hline
\end{tabular}


Table S2. Comparison of the ${ }^{13} \mathrm{C}$ NMR $\left(\mathrm{CDCl}_{3}\right)$ data of natural and synthetic aspidodasycapine

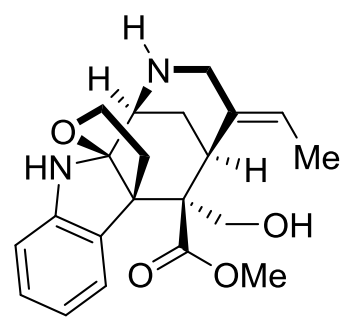

4: aspidodasycarpine

\begin{tabular}{|c|c|c|}
\hline 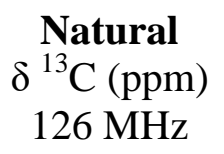 & $\begin{array}{c}\text { Synthetic } \\
\delta{ }^{13} \mathrm{C}(\mathrm{ppm}) \\
101 \mathrm{MHz}\end{array}$ & $\begin{array}{c}\text { Err } \\
\text { (natural-synthetic) } \\
\Delta \delta(\mathrm{ppm})\end{array}$ \\
\hline 174.19 & 174.27 & -0.08 \\
\hline 148.30 & 148.44 & -0.14 \\
\hline 134.34 & 134.40 & -0.06 \\
\hline 130.58 & 130.89 & -0.31 \\
\hline 128.61 & 128.88 & -0.27 \\
\hline 126.12 & 126.14 & -0.02 \\
\hline 123.48 & 123.73 & -0.25 \\
\hline 119.27 & 119.57 & -0.30 \\
\hline 109.17 & 109.50 & -0.33 \\
\hline 104.06 & 104.36 & -0.30 \\
\hline 68.24 & 68.58 & -0.34 \\
\hline 66.91 & 67.47 & -0.56 \\
\hline 57.63 & 57.86 & -0.23 \\
\hline 56.05 & 56.19 & -0.14 \\
\hline 52.99 & 53.23 & -0.24 \\
\hline 51.51 & 51.80 & -0.29 \\
\hline 49.15 & 49.40 & -0.25 \\
\hline 39.05 & 39.15 & -0.10 \\
\hline 31.34 & 32.03 & -0.69 \\
\hline 27.36 & 27.66 & -0.30 \\
\hline 13.17 & 13.34 & -0.17 \\
\hline
\end{tabular}


Table S3. Comparison of the ${ }^{1} \mathrm{H}$ NMR $\left(\mathrm{CDCl}_{3}\right)$ data of natural and synthetic lonicerine

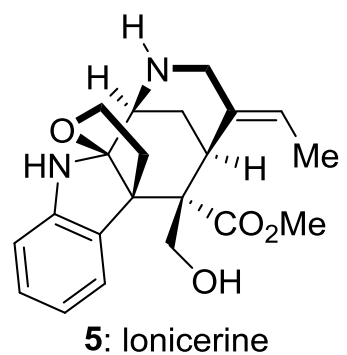

\begin{tabular}{|c|c|c|c|c|}
\hline \multicolumn{2}{|c|}{$\begin{array}{c}\text { Natural } \\
\delta_{\mathrm{H}}[\mathrm{ppm}, \text { mult, } J(\mathrm{~Hz})] \\
400 \mathrm{MHz}\end{array}$} & \multicolumn{2}{|c|}{$\begin{array}{c}\text { Synthetic } \\
\delta_{\mathrm{H}}[\mathrm{ppm}, \text { mult, } J(\mathrm{~Hz})] \\
400 \mathrm{MHz}\end{array}$} & \multirow{2}{*}{$\begin{array}{c}\text { Err } \\
\begin{array}{c}\text { Natural-Synthetic) } \\
\Delta \delta_{\mathrm{H}}(\mathrm{ppm})\end{array} \\
0\end{array}$} \\
\hline 7.07 & $1 \mathrm{H}, \mathrm{d}, 7.5$ & 7.07 & $1 \mathrm{H}, \mathrm{d}, 7.5$ & \\
\hline 6.99 & $1 \mathrm{H}, \mathrm{td}, 7.5,1.0$ & 7.00 & $1 \mathrm{H}, \mathrm{td}, 7.6,0.9$ & -0.01 \\
\hline 6.69 & $1 \mathrm{H}, \mathrm{td}, 7.5,1.0$ & 6.69 & $1 \mathrm{H}, \mathrm{t}, 7.5$ & - \\
\hline 6.53 & $1 \mathrm{H}, \mathrm{d}, 7.5$ & 6.54 & $1 \mathrm{H}, \mathrm{d}, 7.7$ & 0 \\
\hline 5.54 & $1 \mathrm{H}, \mathrm{qd}, 7,2$ & 5.55 & $1 \mathrm{H}, \mathrm{q}, 6.8$ & - \\
\hline 4.44 & $1 \mathrm{H}(N 4 \mathbf{H}), \mathrm{s}$ & 4.36 & $1 \mathrm{H}(N 4 \mathbf{H})$, br s & -0.08 \\
\hline 4.05 & $1 \mathrm{H}, \mathrm{dd}, 8.5,7.3$ & 4.06 & $1 \mathrm{H}, \mathrm{dd}, 8.5,7.3$ & -0.01 \\
\hline 3.87 & $1 \mathrm{H}, \mathrm{d}, 12.5$ & 3.87 & $1 \mathrm{H}, \mathrm{d}, 12.5$ & 0 \\
\hline 3.78 & $1 \mathrm{H}, \mathrm{d}, 12.5$ & 3.78 & $1 \mathrm{H}, \mathrm{d}, 12.5$ & 0 \\
\hline 3.59 & $1 \mathrm{H}, \mathrm{dt}, 13 ., 2$ & 3.59 & $1 \mathrm{H}, \mathrm{dt}, 13.4,2.0$ & 0 \\
\hline 3.42 & $1 \mathrm{H}, \mathrm{ddd}, 13,8.5,4.5$ & $3.47-3.39$ & $1 \mathrm{H}, \mathrm{m}$ & - \\
\hline 3.35 & $1 \mathrm{H}, \mathrm{t}, 3.4$ & 3.37 & $1 \mathrm{H}, \mathrm{s}$ & - \\
\hline 3.27 & $1 \mathrm{H}, \mathrm{d}, 13.5$ & 3.30 & $1 \mathrm{H}, \mathrm{d}, 13.5$ & -0.03 \\
\hline 3.01 & $3 \mathrm{H}, \mathrm{s}$ & 3.01 & $3 \mathrm{H}, \mathrm{s}$ & 0 \\
\hline 2.85 & $1 \mathrm{H}, \mathrm{m}$ & & & \\
\hline 2.85 & $1 \mathrm{H}, \mathrm{m}$ & $2.92-2.76$ & $3 \mathrm{H}, \mathrm{m}$ & - \\
\hline 2.81 & $1 \mathrm{H}, \mathrm{m}$ & & & \\
\hline 2.53 & $1 \mathrm{H}, \mathrm{dd}, 13,4.5$ & 2.53 & $1 \mathrm{H}, \mathrm{dd}, 12.6,4.3$ & 0 \\
\hline 1.78 & $1 \mathrm{H}, \mathrm{dt}, 13.5,3.4$ & 1.80 & $1 \mathrm{H}, \mathrm{dt}, 13.4,3.2$ & -0.02 \\
\hline 1.62 & $1 \mathrm{H}, \mathrm{dd}, 7,2$ & 1.62 & $1 \mathrm{H}, \mathrm{dd}, 6.8,1.8$ & 0 \\
\hline
\end{tabular}


Table S4. Comparison of the ${ }^{13} \mathrm{C}$ NMR $\left(\mathrm{CDCl}_{3}\right)$ data of natural and synthetic lonicerine

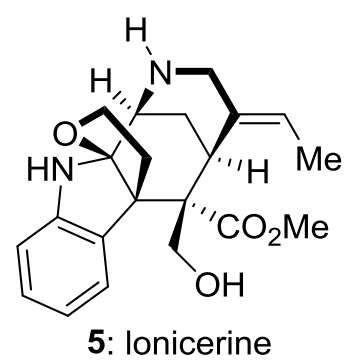

\begin{tabular}{|c|c|c|}
\hline $\begin{array}{c}\text { Natural } \\
\delta{ }^{13} \mathrm{C}(\mathrm{ppm}) \\
101 \mathrm{MHz}\end{array}$ & $\begin{array}{c}\text { Synthetic } \\
\delta{ }^{13} \mathrm{C}(\mathrm{ppm}) \\
126 \mathrm{MHz}\end{array}$ & $\begin{array}{c}\text { Err } \\
\text { (natural-synthetic) } \\
\Delta \delta(\text { ppm })\end{array}$ \\
\hline 176.65 & 176.96 & -0.31 \\
\hline 148.57 & 148.66 & -0.09 \\
\hline 134.32 & 134.54 & -0.22 \\
\hline 133.43 & 133.76 & -0.33 \\
\hline 128.13 & 128.28 & -0.15 \\
\hline 124.38 & 124.55 & -0.17 \\
\hline 122.09 & 122.00 & 0.09 \\
\hline 118.68 & 118.97 & -0.29 \\
\hline 107.33 & 107.54 & -0.21 \\
\hline 102.33 & 102.57 & -0.24 \\
\hline 66.59 & 66.78 & -0.19 \\
\hline 65.18 & 65.40 & -0.22 \\
\hline 57.48 & 57.58 & -0.10 \\
\hline 55.2 & 55.31 & -0.11 \\
\hline 53.06 & 53.35 & -0.29 \\
\hline 51.44 & 51.61 & -0.17 \\
\hline 48.47 & 48.80 & -0.33 \\
\hline 37.41 & 37.54 & -0.13 \\
\hline 33.37 & 33.58 & -0.21 \\
\hline 28 & 28.30 & -0.30 \\
\hline 13 & 13.13 & -0.13 \\
\hline
\end{tabular}

